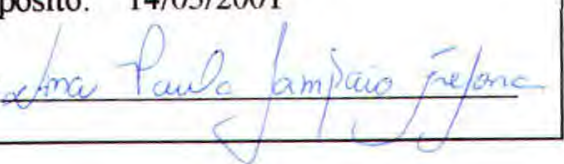

\title{
Aspectos de Geometria Diferencial em Modelos Estatísticos
}

\author{
André Toyofuji Kaneko \\ Orientador: Prof. Dr. Jorge Alberto Achcar
}

Dissertação apresentada ao Instituto de Ciências Matemáticas e de Computação - ICMC-USP, como parte dos requisitos para obtenção do título de Mestre em Ciências de Computação e Matemática Computacional.

USP - São Carlos

Maio/2001 


\section{A Comissão Julgadora:}

Prof. Dr. Jorge Alberto Achcar

Prof. Dr. Dorival Leão Pinto Junior

Profa. Dra. Roseli Aparecida Leandro

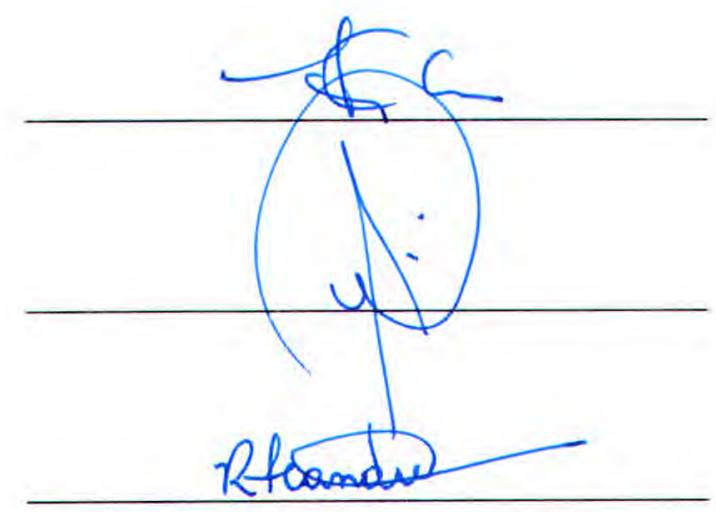




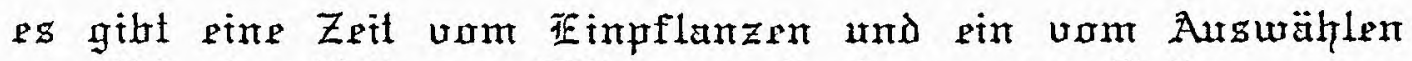

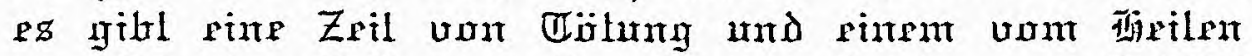

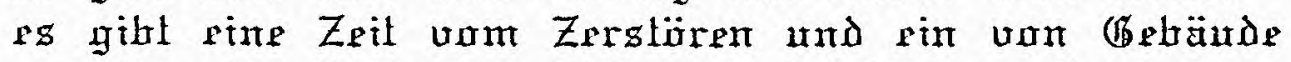

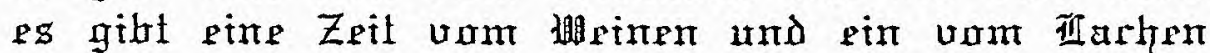

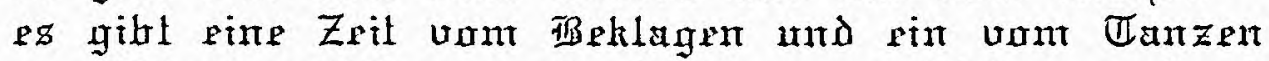

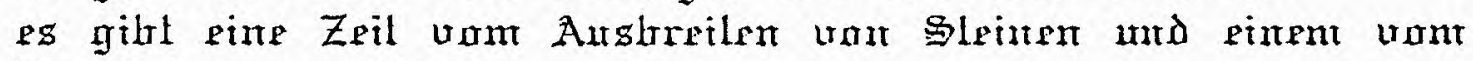

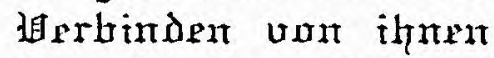

ps gilth pine Zeil uom thntarnten and pin unm Alsplyen unm

Thinarmen

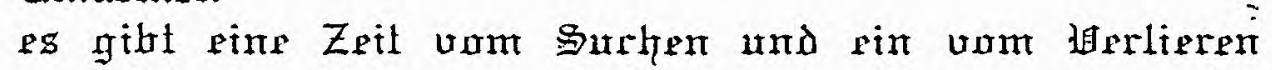

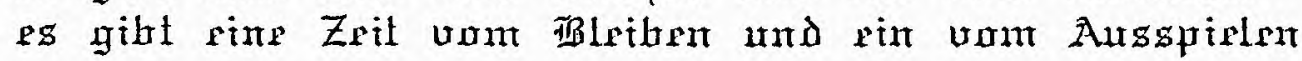

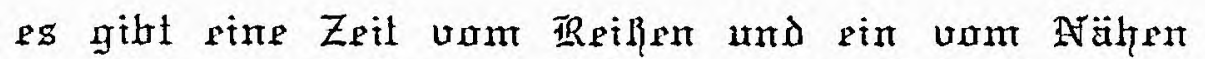

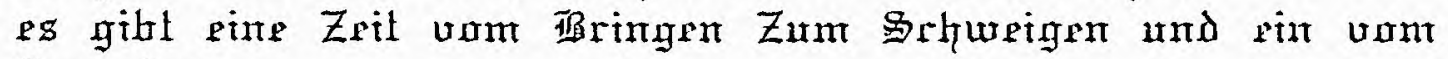

gprerhen

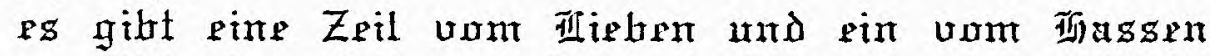

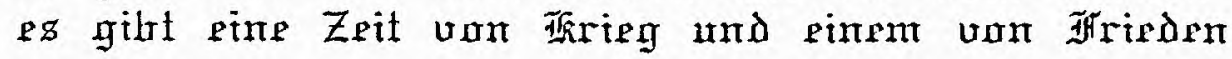

(正rlesiastes 3)

"O homem vive na profunda escuridão...

Dediquei toda minha vida à Ciência e fui capaz de vislumbrar um ou outro lampejo de luz do que seria a verdade, nada mais...."

Marie Curie 


\section{Agradecimentos}

Ao Maior Mistério que fez com que tudo conspirasse na profunda ordem e sincronia.

Ao Prof. Dr. Jorge Alberto Achcar por sua orientação, motivação e grande investimento.

Ao Prof. Dr. Dorival Leão pelo grande apoio, motivação e dedicação à pesquisa.

Ao Prof. Dr. Galvão pelas opiniões de grande valia emitidas na qualificação.

À minha familia.

Ao Amâncio e amigos pelas grandes lições.

Ao pessoal da pós que desburocratizou muita coisa, em especial à paciência da Laura e Beth. A Fapesp. 


\section{Resumo}

Nesta dissertação de mestrado, sumarizamos alguns conceitos básicos de geometria diferencial e estudamos a conexão existente entre geometria diferencial e modelos estatísticos. Assim, calculamos medidas geométricas associadas aos modelos estatísticos e estudamos os efeitos de uma boa parametrização nas inferências obtidas. Na nova parametrização, verificamos se a precisão dos resultados da inferência melhoram e quais são as relações existentes com as medidas geométricas.

Várias aplicações são consideradas, especialmente com modelos para dados de sobrevivência censurados ou não e modelos não-lineares.

Também estudamos os efeitos de uma parametrização em inferência Bayesiana, especialmente usando algoritmos de simulação de amostras MCMC (Monte Carlo em Cadeias de Markov). 


\begin{abstract}
In this work, we studied some basic concepts of geometry diferencial and we studied the existent connection between geometry diferencial and statistical models. Thus, we calculated geometric measures associated to the statistical models and we studied the effects of a good parametrização in the obtained inferences. In the new parametrização, we were verified the precision of the results of the inference they improve and which are the existent relationships with the geometric measures.

Several applications are considered, especially with models for censored survival data or not and no linear models.

We also studied the effects of a parametrização in inference Bayesiana, especially using algorithms of simulation of samples MCMC (Monte Carlo in Chains of Markov).
\end{abstract}




\section{Índice}

Capítulo1:Introdução......................................................1 Capítulo2:Modelos.Estatísticos.....................................5

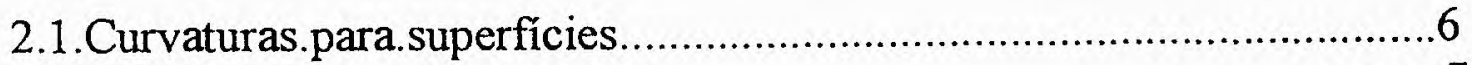

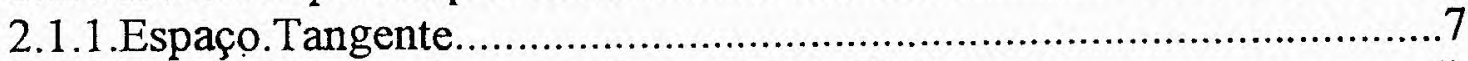

2.1.2.Métrica.Riemaniana.e.Informação.de.Fisher.................................... 7

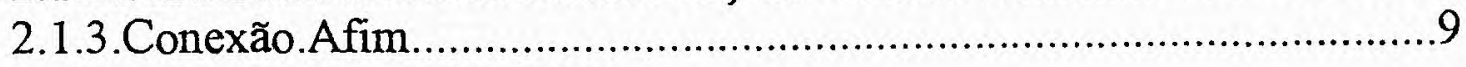

Capítulo3:Algumas.Aplicações. Numéricas................13

3.1.Conexão.entre.aproximação.de.Laplace.e.tensores.associados...........13

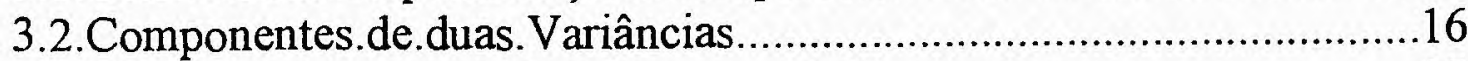

3.3.Conexão.entre.tensor.de.Riemann.e.dados.censurados.......................17

3.4.Comportamento.do.escalar.de.Ricci.diante.da.ortogonalização.dos.pa

3.5.Reparametrização.para.a.distribuição.de.valor.Extremo......................21

3.6.Comportamento.do.escalar.de.Ricci.em.uma.análise.Bayesiana.usando

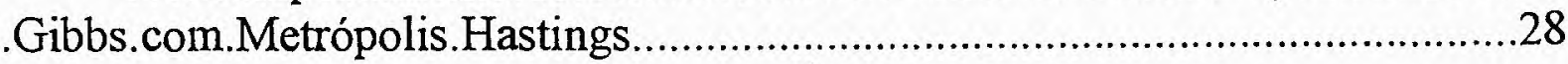

Capítulo4:.Modelos.Não.Lineares................................36

4.1.Curvaturas.para.dimensões.maiores.que.dois....................................36

4.2.Uso.de.curvaturas para.discriminação.de.modelos.de.crescimento.sig móide. 36

4.3.Casos.de.Reparametrização.em.modelos.não.lineares........................50

Capítulo5:Estudos.de.Curvaturas.de.distribuições.Per filadas.

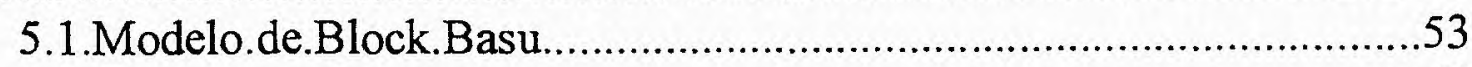

5.2..Reparametrizações......................................................................53

5.3.Relação.entre.a.curvatura.global.do.modelo.três.com.parâmetros.e.as. curvaturas.das.distribuições.perfiladas.com.dois.parâmetros.

Capítulo6:Conclusões.e.Prespectivas.Futuras............71

Apêndices.........................................................................72

Apêndice1.Vetores.Contravariantes.Covariantes...... 72 Apêndice2.Expressões.dos.tensores.para.o.caso.bidime nsional.geral......................................................................78 Apêndice3.Método.de.Laplace.para.aproximação.de Integrais. 
Apêndice4.Medidas.de.Curvaturas.de.Batts.e.Watts. e.profiles.t-plots.......................................................................80 Apêndice5.Medidas.de.Não.Linearidade....................81

Bibliografia.....................................................................82 


\section{Capítulo 1 \\ Introdução}

Nesta dissertação introduzimos uma conexão entre geometria diferencial e modelos estatísticos. Esses modelos estatísticos são parametrizados por quantidades desconhecidas $\theta$.

$\mathrm{O}$ uso de geometria diferencial e sua conexão com modelos estatísticos tem sido estudado por vários autores (ver, por exemplo, Barndorff-Nielsen, 1986).

Em geral, a escolha de uma parametrização é arbitrária e uma transformação de $\theta$ pode levar à melhores resultados inferenciais (ver por exemplo, Anscombe, 1964, Sprott, 1973, 1980; ou Achcar e Smith, 1990).

O problema da boa parametrização é objetivo de pesquisa intensa entre os estatísticos. Parametrização para melhorar a "normalidade" da função de verossimilhança quando o tamanho da amostra não é grande é um dos objetivos de muita pesquisa estatística (ver por exemplo, Sprott, 1973,1980). Outra possibilidade é encontrar parametrizações ortogonais (ver Cox e Reid, 1987).

Em muitas situações uma boa parametrização pode ser avaliada a partir de medidas de curvatura usando conceitos de geometria diferencial (ver por exemplo, Bates e Watts, 1992, Kass e Slate, 1992).

Nesta dissertação de mestrado, sumarizamos alguns conceitos básicos de geometria diferencial e estudamos a conexão existente entre geometria diferencial e modelos estaísticos. Assim, calculamos medidas geométricas associadas aos modelos estatísticos e estudamos os efeitos de uma boa parametrização nas inferências obtidas. $\mathrm{Na}$ nova parametrização, verificamos se a precisão dos resultados inferenciais melhoram e quais são as relações existentes com as medidas geométricas.

Várias aplicações são consideradas, especialmente com modelos para dados de sobrevivência censurados ou não e modelos não-lineares.

Também estudamos os efeitos de uma parametrização em inferência Bayesiana, especialmente usando algoritmos de simulação de amostras MCMC (Monte Carlo em Cadeias de Markov).

A escolha da parametrização de um modelo estatístico é de grande importância em todos os estágios da análise estatística. A eficiência de métodos numéricos usadas na estimação e confiabilidade de inferências baseadas em aproximações para grandes amostras são muito sensíveis a parametrização. A pesquisa contida nessa dissertação de mestrado contribui para entender alguns efeitos de reparametrizações e o comportamento de uma nova medida de curvatura diante de tais reparametrizações.

Obter o modelo estatístico para uma situação de interesse não é o bastante em muitas situações, pois é necessário que a precisão das estimativas dos parâmetros permitam conclusões confiáveis. Exceto para modelos muito simples, regiões de confiança ou de credibilidade requerem muito custo computacional. Além disso, a menos que os parâmetros sejam independentes ou a verossimilhança ou a posteriori sejam de fato normais, a mudança no valor de um parâmetro afeta a inferência dos outros parâmetros de módo muito complexo. Além disso, os métodos usados para obter regiões exatas para um modelo não são aplicáveis a outros modelos. Por isso, usa-se teoria assintótica. Desde que a distribuição assintótica usada normalmente é a normal, que é completamente determinada pela média e variância, análises inteiras são reduzidas a estimativa pontual e do desvio padrão. Todas inferências são baseadas nesses valores, dando-se pouca atenção à validade ou não das aproximações assintóticas. Aplicações descuidadas desses resultados podem ser perigosas.

A aplicabilidade de tais resultados assintóticos são muito ampliados pela reparametrização adequada. Uma reparametrização cuidadosa pode colocar a verossimilhança muito próxima da normal. Dessa forma, as inferências baseadas na normalidade assintótica dos estimadores de máxima verossimilhança podem ser muito acuradas. Bayesianos podem 
escolher reparametrizações para que a posteriori seja normal, aumentando a confiabilidade das suas inferências baseadas na normalidade assintótica da posteriori.

Em problemas multiparamétricos, inferências exatas podem ser muito dificeis e os resultados de reparametrização podem ser drásticos. Inferências com amostras pequenas em regressões não lineares são particularmente dificeis e se usa aproximações lineares da superficie esperada. Há casos, todavia, em que essas aproximações são péssimas. Novamente, a escolha apropriada da parametrização pode melhorar tais aproximações e os resultados da inferência.

Esses modelos não lineares encontram muita aplicação na indústria. Pesquisadores trabalham com parâmetros que possuem significado físico. Pelas reparametrizações que levam à normalidade, desvios padrões podem ser obtidos para as estimativas dos parâmetros. Com esses valores, o pesquisador pode avaliar melhor seus valores experimentais.

Kass e Slate (1992), estabeleceram uma conexão entre uma medida de curvatura (medida de Kass e Slate) e a normalidade da respectiva distribuição estatística. Motivados por esse resultado, propomos uma nova medida de curvatura, o escalar de Ricci e analisamos seu comportamento em várias situações de interesse estatístico. Sendo que os casos de maior interesse são os que envolvem reparametrizações do modelo estatístico levando a melhor normalidade da distribuição em questão. Esse é o objetivo desse trabalho.

O escalar de Ricci foi eficiente em captar a parametrização mais próxima da normalidade conforme mostra, por exemplo, o caso da reparametrização para a distribuição de valor extremo. Considere o modelo estatístico para o tempo de falha:

$\mathrm{Y}=\ln (\mathrm{T})=\mu+\sigma \mathrm{W}$

A parametrização é dada por

$\varphi=(\mu, \sigma)$

Uma reparametrização pode ser dada por

$\psi=\left(\theta_{1}, \theta_{2}\right)$

onde

$\theta_{1}=\ln (\sigma)$

$\theta_{2}=(\mu+\mathrm{a}) / \sigma$

Os escalares de Ricci estão na tabela 1.1 e se referem a um exemplo que será tratado em detalhes no capítulo 3:

\begin{tabular}{|l|l|l|}
\hline Parametrização & $\mathbf{R}_{1212}$ & $\mathrm{k}$ \\
\hline$(\mu, \sigma)$ & 220106.1448 & 249878.9541 \\
\hline$\left(\theta_{1}, \theta_{2}\right)$ & 20.61205207 & 22.82734875 \\
\hline
\end{tabular}

Tabela 1.1: valores de $R_{1212}$ e k para parametrizações $(\mu, \sigma)$ e $\left(\theta_{1}, \theta_{2}\right)$

Os contornos para a função de verossimilhança para $\left(\theta_{1}, \theta_{2}\right)$ mostram a grande normalidade nessa parametrização, na parametrização $(\mu, \sigma)$ tal fato não se dava. $O$ escalar de Ricci se mostrou menor na melhor parametrização.

Tais comentários estão ilustrados nas figuras 1.,1.2,1.3,1.4. 


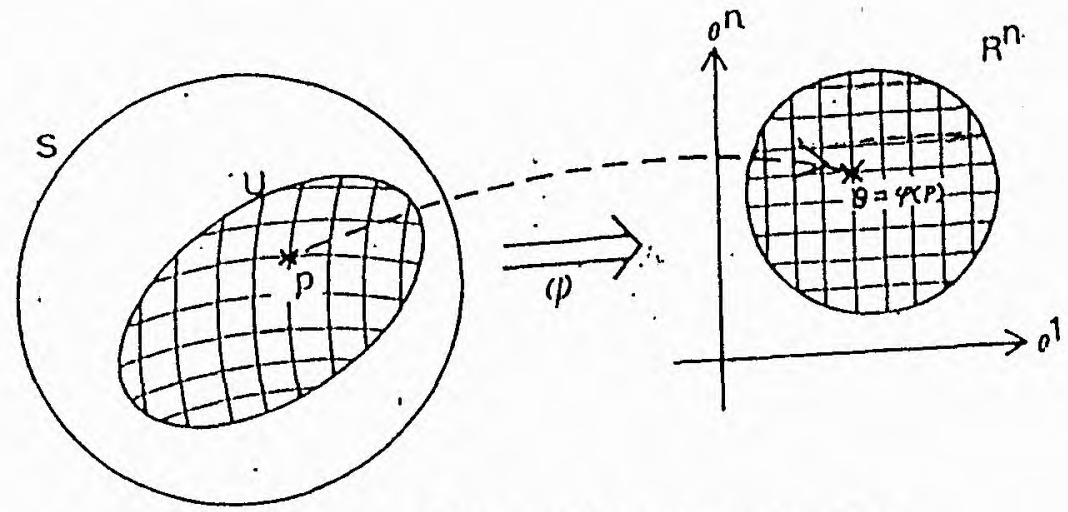

Figura 1.1.parametrização $\varphi$

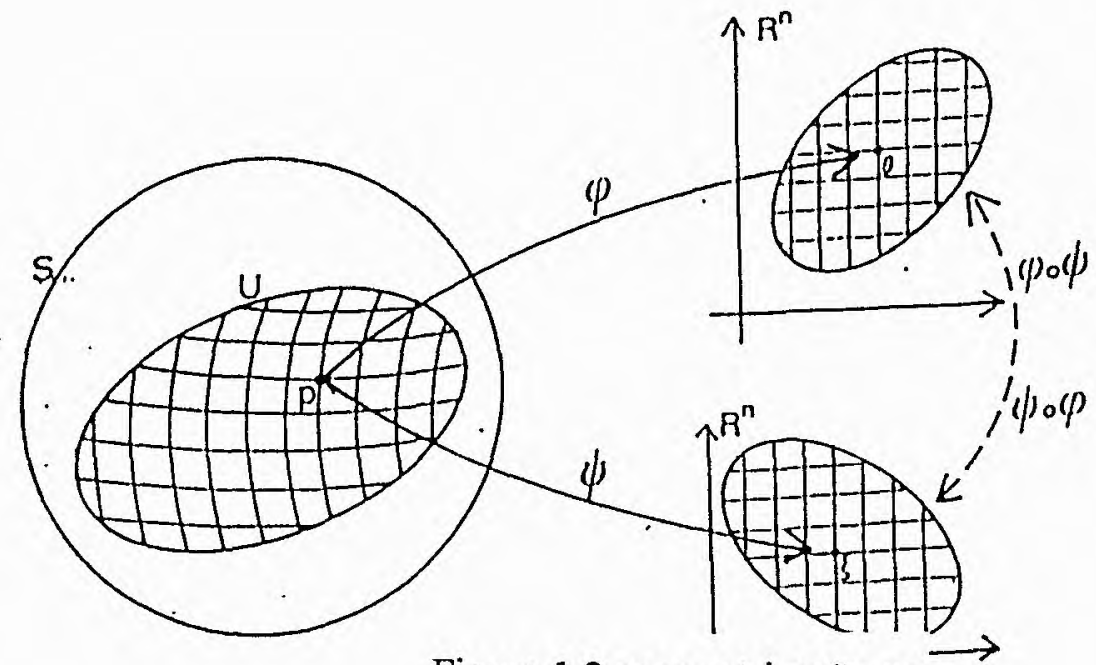

Figura 1.2.parametrização $\psi$ 


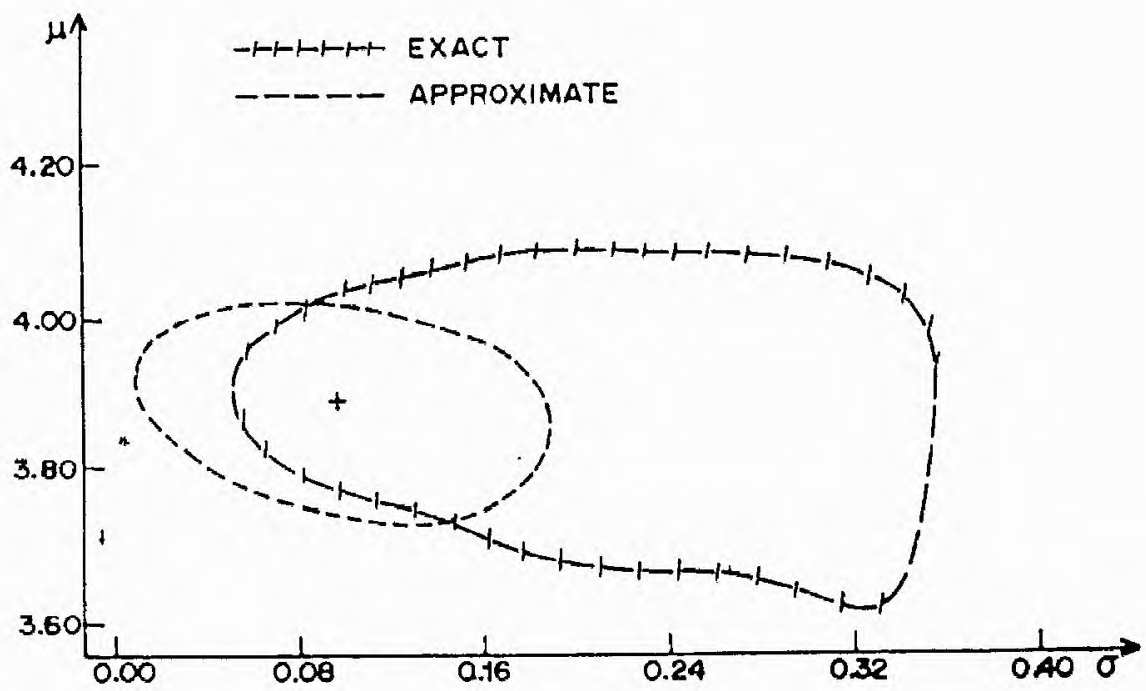

Figura 1.3. contornos da função de verossimilhança para $\mu$ e $\sigma(l(\mu, \sigma)=-1)$

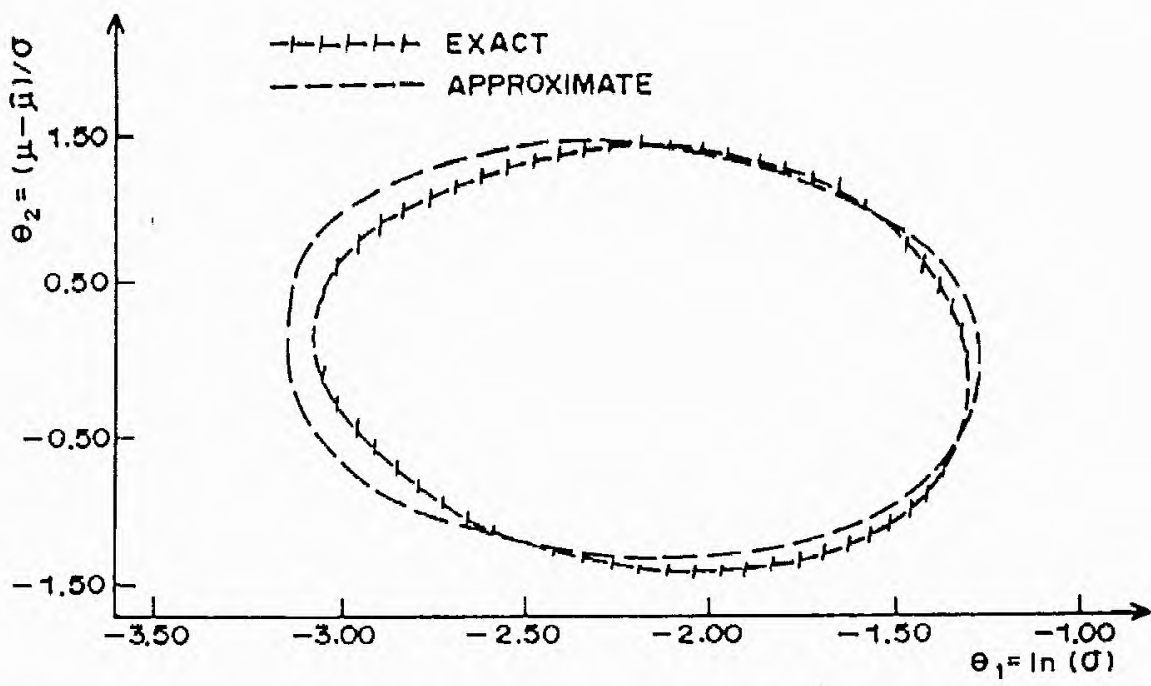

Figura 1.4. contornos da função de verossimilhança para $\theta_{1}$ e $\theta_{2}\left(1\left(\theta_{1}, \theta_{2}\right)=-1\right)$ 


\section{Capítulo 2 \\ Modelos Estatísticos}

Considere $(\Omega, \beta(R))$ um espaço mensurável e uma familia de probabilidade $P_{\theta}$ indexadas por $\theta \in \Theta \subseteq R^{m}$, onde $\Theta$ é o espaço paramétrico. Vamos considerar que existe uma probabilidade $\lambda$ definida sobre $(R, \beta(R))$, tal que as probabilidades $P_{\theta}$ sejam absolutamente contínuas com relação a uma medida $\lambda$ sobre $(R, \beta(R))$ :

$\mathrm{P}_{\theta}<\lambda, \forall \theta \in \Theta$

Assim, para todo $\theta \in \Theta$, existe $f: R \rightarrow[0, \infty)$ mensurável tal que

$P_{\theta}(B)=\int_{B} f(x) \lambda d x$

$\forall \mathrm{B} \in \beta(\mathrm{R})$

Desta forma, obtemos uma família de funções densidade $\{f():. \theta \in \Theta\}$ parametrizadas por $\theta$. Tal família caracteriza o modelo estatistico.

Alguns exemplos :a) Considere uma distribuição Normal Univariada com parametrização $\theta=(\mu, \sigma)$. O espaço paramétrico é dado por, $\Theta=\{(\mu, \sigma): \mu \in R, \sigma>0\}=R \times R_{+} \subset R^{2}$ A função densidade de probabilidade para $X(x>0)$ é dada por

$f(x)=1 /(2 \pi)^{1 / 2} \exp \left[(-1 / 2 \sigma)(x-\mu)^{2}\right]$

b)Distribuição Gama com parametrização $\theta=(\alpha, \beta)$ e espaço paramétrico $\Theta=\left\{(\mu, \beta): \mu \in R_{+}, \beta \in R_{+}\right\}=R_{+} x R_{+} \subset R^{2}$

A fdp para $X(x>0)$ é dada por,

$f(x)=(\beta / \mu)^{\beta}(x)^{\beta-1} / \Gamma(\beta) \exp (-x \beta / \mu)$

c)Distribuição Binomial Negativa, seja $X$ uma variável aleatória com fdp, $X(T)=\{0,1,2, \ldots\}=N$

$f(x)=(r+x-1) ! /[x !(r-1) !] p^{r} q^{x}$

$0<\mathrm{p}<1$

$q=1-p$

$\mathrm{x}=0,1,2, \ldots$

No caso dominado (absolutamente contínuo ou discreto), o modelo estatístico é definido por $S=\left\{f_{\theta}(x) ; \theta \in \Theta\right.$ e $\left.x \in R^{n}\right\}$ e para o caso absolutamente contínuo e $S=\left\{P_{\theta}(x) ; \theta \in \Theta\right.$ e $\left.x \in N^{m}\right\}$ para o caso discreto. Em geral, tanto no modelo absolutamente contínuo quanto no discreto o espaço paramétrico é contínuo. Em termos de Inferência Estatística, diferentes parâmetros definem diferentes pontos em S. Por exemplo, duas distribuições Normais Univariadas com mesmas médias $\mu$, mas com desvios padrões $\sigma_{1}$ e $\sigma_{2}$ diferentes são representadas pelos pontos $\left(\mu, \sigma_{1}\right)$ e $\left(\mu, \sigma_{2}\right)$ que são diferentes. No entanto, o mesmo não ocorre quanto a $\mathrm{x}$, pois podemos ter $\mathrm{x}$ 's (conjunto de dados) diferentes que conduzem a mesmos parâmetros.

Caso as funções $f_{\theta}$ sejam suficientemente suaves, é possível introduzirmos uma estrutura de diferenciabilidade sobre 0 modelo estatístico, ou seja, sobre a família $\left\{f_{\theta}(x): \theta \in \Theta\right\}, \forall \theta \in \Theta$.

Sendo $S_{x}$ a secção de $S$ num ponto $x$ fixo, $S=\left\{f_{\theta}(x): \theta \in \Theta, x \in \Omega\right\}$, definir uma função $\phi_{\mathrm{x}}: \mathrm{S}_{\mathrm{X}} \rightarrow \mathrm{R}^{\mathrm{n}}$ por 


$$
\phi\left[\mathrm{f}_{\theta}(\mathrm{x})\right]=\theta, \forall \theta \in \Theta .
$$

Assim na distribuição Normal Univariada, $\phi_{\mathrm{x}}$ é dada por $\phi=(\mu, \sigma)$; na distribuição Weibull, temos $\phi=(\alpha, \beta)$; na distribuição Binomial Negativa, temos $\phi=(p)$.

Uma função $h$ é dita ser um homeomorfismo se

a)h é bijetora

b)h e sua inversa $\mathrm{h}^{-1}$ são contínuas.

A função $\phi: S \rightarrow O$ é um homeomorfismo onde $O \subset R^{\mathrm{n}}$ é aberto.

No caso das distribuições estatísticas analisadas, $\phi$ obedece as condições da definição de homeomorfismo: é bijetora pois dado um modelo estatístico temos apenas um conjunto de parâmetros que o define e vice-versa. A função é contínua em decorrência de possuírem todas as derivadas parciais (2.3.b), o que garante a continuidade.

Esse homeomorfismo $\phi$ é chamado função coordenada; e define a parametrização sob a qual o modelo se encontra.

Podemos definir diversos sistemas de coordenadas para o modelo estatístico. Todos esses diferentes sistemas de coordenadas se referem ao mesmo modelo estatistico. Seja $\Psi$ um outro sistema de coordenadas para o modelo estatístico, se $\Psi$ puder ser expressa como função do sistema anterior de coordenadas, dizemos que foi realizada uma reparametrização do modelo estatístico.

Uma reparametrização bastante utilizada no caso da distribuição Normal Univariada é a de parâmetros Naturais:

$$
\begin{aligned}
& \phi=\left(\phi_{1}, \phi_{2}\right) \\
& \phi_{1}=\mu / \sigma^{2} \\
& \phi_{2}=1 /\left(2 \sigma^{2}\right)
\end{aligned}
$$

Dependendo de quais questões estamos interessados em resolver, a parametrização usada torna-se fundamental. Como ilustração considere uma análise Bayesiana, onde o interesse é encontrar distribuições a posteriori próximas à normal. A pergunta natural que surge é qual reparametrização poderia levar a tal resultado.

\subsection{Curvaturas para superfícies} são

As condições requeridas para a aplicação da teoria geométrica nos modelos estatísticos

a) Todas as $p(x, \theta)$ devem ter um suporte comum tal que $p(x, \theta)>0$ para todos os $x \in X$, onde $X$ é o suporte.

b) Seja $1(x, \theta)=\ln p(x, \theta)$. Para todo $\theta$ fixado, $n$ funções em $x$

$\partial \mathrm{l}(\mathrm{x}, \theta) / \partial \theta^{\mathrm{i}}$ são linearmente independentes.

c)Os mornentos das variáveis aleatórias $\left(\partial / \partial \theta^{i}\right) 1(x, \theta)$ existem até as ordens necessárias.

d)As derivadas parciais $\partial / \partial \theta^{\mathrm{i}}$ e a integração com respeito a medida $\mathrm{P}$ pode ser intercambiada como

$\partial / \partial \theta^{i} \int f(x, \theta) \mathrm{dP}=\int \partial / \partial \theta^{i} f(x, \theta) d P$ 
para quaisquer funções $f(x, \theta)$ a serem tratadas.

\subsubsection{Espaço tangente}

$O$ espaço tangente $T_{p}$ no ponto $P$ é o espaço vetorial gerado pelos.n vetores tangentes $\partial_{\mathrm{j}}$ à superficie no ponto $\mathrm{P}$. Trata-se de uma linearização local da superfície ao redor do ponto P.

Como os n vetores $\partial_{\mathrm{j}}$ são linearmente independentes (condição de regularidade b), tais vetores formam uma base do espaço tangente $T_{p}$. $\dot{E}$ chamada base natural associada ao sistema de coordenadas $\theta$.

Dessa forma, qualquer vetor tangente $A \in T_{p}$. pode ser representado como combinação linear de $\partial_{\mathrm{j}}$ :

$\mathrm{A}=\sum_{\mathrm{j}=1}^{\mathrm{n}} \mathrm{A}^{\mathrm{j}} \partial_{\mathrm{j}}$

ou na notação de Einstein (notação da Análise Tensorial que omite o sinal de somatório e pressupõe somar sobre os índices repetidos)

$A=A^{j} \partial_{j}$

Alguns exemplos:

1)Distribuição Normal: A função de verossimilhança para $\theta=(\mu, \sigma)$ é dada por, $L(x ; \sigma)=\Pi_{j=1}{ }^{n} f\left(x_{j}\right)=\Pi_{j=1}{ }^{n} 1 /(2 \pi \sigma)^{1 / 2} \exp \left[-1 /\left(2 \sigma^{2}\right)\left(x_{j}-\mu\right)^{2}\right]$

Assim, $1=\ln L(x ; \sigma)=-1 / 2 \ln (\sigma)+\sum_{j=1}{ }^{n}-\left(x_{j}-\mu\right)^{2} /\left(2 \sigma^{2}\right)+$ constante

Dai, temos:

$1_{\mu}=\sum_{\mathrm{j}=1}{ }^{\mathrm{n}}-\left(\mathrm{x}_{\mathrm{j}}-\mu\right) /\left(2 \sigma^{2}\right)$

$l_{\sigma}=-1 /(2 \sigma)+\sum_{j=1}^{n}\left(x_{j}-\mu\right)^{2} /\left(\sigma^{3}\right)$

2)Distribuição Gama. A função de verossimilhança para $\mu$ e $\beta$ é dada por,

$L(x ; \mu, \beta)=\prod_{j=1}^{n} f\left(x_{j}\right)=\prod_{j=1}^{n}(\beta / \mu)^{\beta}\left(x_{j}\right)^{\beta-1} / \Gamma(\beta) \exp \left(-x_{j} \beta / \mu\right)$

Daí,

$l=\ln L(x ; \mu, \beta)=n \beta / \mu \ln (\beta / \mu)-n \ln [\Gamma(\beta)]+(\beta-1) \sum_{j=1}{ }^{n} \ln \left(x_{j}\right)-\sum_{j=1}{ }^{n}\left(x_{j} \beta / \mu\right)$

Assim,

$l_{\alpha}=-n \beta / \mu+\sum_{j=1}{ }^{n} x_{j} \beta / \mu^{2}$

$l_{\beta}=n \ln (\beta / \mu)+n+\sum_{j=1}{ }^{n} \ln \left(x_{j}\right)-\partial[\Gamma(\beta) / \partial \beta] / \Gamma(\beta)-\sum_{j=1}{ }^{n} x_{j} / \mu$

3)Distribuição Binomial Negativa. A função de verossimilhança para $\mathrm{p}$ é dada por, $L(x ; p)=\prod_{j=1}^{n} f\left(x_{j}\right)=\Pi_{j=1}^{n}(r+x-1) ! /[x !(r-1) !] p^{r} q^{x}$, onde $q=1-p$

Assim, o logaritmo da verossimilhança é dado por,

$\mathrm{l}=\ln \mathrm{L}(\mathrm{x} ; \mathrm{p})=\mathrm{nr} \ln (\mathrm{p})+\left(\sum_{\mathrm{j}=1}{ }^{\mathrm{n}} \mathrm{x}_{\mathrm{j}}\right) \ln (1-\mathrm{p})+$ constante

Dai,

$1_{p}=n r / p-\left(\sum_{j=1}^{n} x_{j}\right) /(1-p)$

Notamos que a dimensão do espaço tangente das duas primeiras distribuições é dois e o da terceira distribuição é um. Distribuições com espaço tangente unidimensional não podem ser analisadas pelo cálculo tensorial de superfícies.

\subsubsection{Métrica Riemaniana e Informação de Fisher}


Se introduzirmos o produto interno $<A, B>$ de dois vetores tangentes $A, B$ em $T_{p}, a$ superficie $S$ é chamada de espaço Riemaniano com o produto interno.

$<\mathrm{A}, \mathrm{B}\rangle=\mathrm{E}[\mathrm{A}(\mathrm{x}) \mathrm{B}(\mathrm{x})]$

A fórmula (2.5) é uma forma natural de se definir o produto interno.

$O$ produto interno de dois vetores da base $\partial_{\mathrm{i}}$ e $\partial_{\mathrm{j}}$ é dado por

$\mathrm{g}_{\mathrm{ij}}(\theta)=<\partial_{\mathrm{i}}, \partial_{\mathrm{j}}>=\mathrm{E}\left[\partial_{\mathrm{i}} \mathrm{l}(\mathrm{x}, \theta), \partial_{\mathrm{j}} \mathrm{l}(\mathrm{x}, \theta)\right]$

Essas $\mathrm{n}^{2}$ quantidades $\mathrm{g}_{\mathrm{j}}(\theta), \mathrm{i}, \mathrm{j}=1, \ldots, \mathrm{n}$ compõe um objeto chamado tensor métrico.

Alguns exemplos:

1)Distribuição Normal. As segundas derivadas do logaritmo da verossimilhança para $\mu$ e $\sigma$ são dados por,

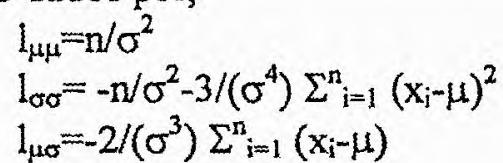

Isto é,

$\mathrm{g}_{11}=\mathrm{E}\left\{-1_{\mu \mu}\right\}=\mathrm{n} / \sigma^{2}$

$\mathrm{g}_{22}=\mathrm{E}\left\{-\mathrm{l}_{\sigma \sigma}\right\}=2 \mathrm{n} / \sigma^{2}$

$\mathrm{g}_{12}=\mathrm{g}_{21}=\mathrm{E}\left\{-\mathrm{I}_{\mu \mathrm{\sigma}}\right\}=0$

Portanto, a matriz de informação de Fisher é dada por

$$
\begin{aligned}
& \mathrm{g}_{11}=-\mathrm{n} / \sigma^{2} \\
& \mathrm{~g}_{12}=\mathrm{g}_{21}=0 \\
& \mathrm{~g}_{22}=2 \mathrm{n} / \sigma^{2}
\end{aligned}
$$

2)Distribuição Gama. Considere a distribuição gama com densidade dada por $f(x ; \mu, \beta)=(\beta / \mu)^{\beta} x^{\beta-1} \exp (-x \beta / \mu)$

Aqui temos,

$1_{\mu \mu}=-n / \mu+1 / \mu^{2} \sum_{i=1}^{n}\left(x_{i}\right)$

$I_{\mu \beta}=-n / \mu+\Sigma^{n}{ }_{i=1}\left(x_{i}\right) / \mu^{2}$

$I_{\beta \beta}=n / \beta-\Gamma_{\beta \beta}(\beta) / \Gamma(\beta)+\Gamma_{\beta}^{2}(\beta) / \Gamma^{2}(\beta)$

Considerar a distribuição gama $(\mu, \beta)$ dada na seção 2.1.3. Considerando apenas uma observação, temos,

$$
\begin{aligned}
& \mathrm{g}_{11}=\mathrm{E}\left\{-\mathrm{l}_{\mu \mu}\right\}=2 \mathrm{n} \beta / \mu^{2} \\
& \mathrm{~g}_{22}=\mathrm{E}\left\{-\mathrm{l}_{\sigma \sigma}\right\}=0 \\
& \mathrm{~g}_{12}=\mathrm{g}_{21}=\mathrm{E}\left\{-\mathrm{I}_{\mu \sigma}\right\}=\phi(\beta) \\
& \text { onde } \phi(\beta)=[\ln \Gamma(\beta)]_{\beta \beta}-1 / \beta
\end{aligned}
$$

Portanto, a matriz de informação de Fisher é dada por $I=\left\{\left(\mathrm{g}_{i j}\right)\right\}, \mathrm{i}, \mathrm{j}=1,2$, onde

$$
\begin{aligned}
& \mathrm{g}_{11}=2 \mathrm{n} \beta / \mu^{2} \\
& \mathrm{~g}_{12}=\mathrm{g}_{21}=0
\end{aligned}
$$


$\mathrm{g}_{22}=\phi(\beta)$

\subsubsection{Conexão Afim}

Supor agora que queremos associar a cada ponto $\theta \in S$, um vetor $A(\theta) \in T_{\theta}$. Essa coleção $A=\{A(\theta) \mid \theta \in S\}$ é chamado campo vetorial.

Um campo vetorial A pode ser representado na forma de componentes como

$$
A=A^{j}(\theta) \partial_{j}(\theta)
$$

Dado um campo vetorial em $\mathrm{S}$, temos interesse em compararmos tal campo em dois pontos distintos $\theta^{\prime}$ e $\theta$ de $S$.

Os espaços tangentes $T_{\theta}$ e $T_{\theta}$, são diferentes, não havendo forma direta de compararmos $A(\theta)$ e $A\left(\theta^{\prime}\right)$. Essa diferença ocorre porque as bases $\partial_{j}(\theta)$ e $\partial_{j}\left(\theta^{\prime}\right)$ são diferentes.

Para comparamos $A(\theta)$ e $A\left(\theta^{\prime}\right)$, considere uma aplicação linear $m: T_{\theta+d \theta} \rightarrow T_{\theta}$, entre dois espaços tangentes de pontos próximos por um infinitésimo. Tal aplicação depende de d $\theta$ e se reduz à aplicação identidade quando $d \theta$ tende a zero. $\partial_{\mathrm{j}}(\theta)$.

Desde que d $\theta$ é pequeno, o versor $\partial_{j}^{\prime}=\partial_{j}(\theta+d \theta) \in T_{\theta+d \theta}$ é aplicado em $m\left(\partial_{j}{ }_{j}\right)$ perto de

Seja $\Delta \partial_{j}$ a diferença entre $m\left(\partial_{j}^{\prime}\right)$ e $\partial_{j}$ :

$\Delta \partial_{\mathrm{j}}=\mathrm{m}\left(\partial_{\mathrm{j}}{ }_{\mathrm{j}}\right) \partial_{\mathrm{j}} \in \mathrm{T}_{\theta}$

negligenciando-se termos de ordem superior a um.

$\Delta \partial_{\mathrm{j}}$ pode ser expresso como

$\Delta \partial_{\mathrm{j}}=\mathrm{d} \theta^{\mathrm{i}} \Gamma_{\mathrm{ij}}^{\mathrm{k}}(\theta) \partial_{\mathrm{k}}$

onde $\mathrm{d} \theta^{\mathrm{i}} \Gamma_{\mathrm{ij}}{ }^{\mathrm{k}}(\theta)$ são componentes de $\Delta \partial_{\mathrm{j}} \in \mathrm{T}_{\theta}$, são a representação de $\Delta \partial_{\mathrm{j}}$ no espaço tangente $T_{\theta}$,

Essas $n^{3}$ funções $\Gamma_{\mathrm{ij}}{ }^{\mathrm{k}}(\theta)$ são chamadas coeficientes da conexão afim porque $m$ fornece uma correspondência afim entre $T_{\theta}$ e $T_{\theta+d \theta}$.

A métrica do tensor de Riemann $g_{i j}(\theta)$ cria uma conexão afim natural, cujos coeficientes são dados por

$$
[\mathrm{ij} \mathrm{k}]=1 / 2\left[\partial_{\mathrm{i}} \mathrm{g}_{\mathrm{jk}}+\partial_{\mathrm{j}} \mathrm{g}_{\mathrm{ik}}-\partial_{\mathrm{k}} \mathrm{g}_{\mathrm{ij}}\right]
$$

Associado a esses símbolos de Christofell temos o tensor de curvatura de Riemann, dado por (Amari 1982)

$$
\mathrm{R}_{\mathrm{ijkm}}=\left(\partial_{\mathrm{i}} \Gamma_{\mathrm{jk}}{ }^{\mathrm{s}}-\partial_{\mathrm{j}} \Gamma_{\mathrm{ik}}{ }^{\mathrm{s}}\right) \mathrm{g}_{\mathrm{sm}}+\left(\Gamma_{\mathrm{irm}} \Gamma_{\mathrm{jk}}{ }^{\mathrm{r}}-\Gamma_{\mathrm{jmm}} \Gamma_{\mathrm{jk}}{ }^{\mathrm{T}}\right)
$$

e o escalar de Ricci ou curvatura seccional dada por:

$k=-g^{i k} g^{j m} R_{i j k m}$

Para casos unidimensionais pode-se usar a curvatura geométrica canônica, dada por

$\mathrm{K}=\mathrm{g}_{11} /\left(1+\left(\mathrm{g}_{1}\right)^{1 / 2}\right)^{3 / 2}$ 
2.1.4.Exemplos

1)Distribuição Normal

Para a distribuição Normal, os elementos da inversa de Fisher são dados por

$\operatorname{det} g=-2 n^{2} / \sigma^{4}$

$\mathrm{g}^{11}=\mathrm{g}_{22} /$ det $\mathrm{g}=-\sigma^{2} / \mathrm{n}$

$\mathrm{g}^{22}=\mathrm{g}_{11} /$ det $\mathrm{g}=-\sigma^{2} / 2 \mathrm{n}$

$\mathrm{g}^{12}=\mathrm{g}^{12}=-\mathrm{g}_{12} /$ det $\mathrm{g}=0$

As derivadas de $\mathrm{g}_{\mathrm{ij}}$ são dadas por:

$$
\begin{aligned}
& \mathrm{g}_{111}=0 \\
& \mathrm{~g}_{112}=2 \mathrm{n} / \sigma^{3} \\
& \mathrm{~g}_{221}=0 \\
& \mathrm{~g}_{222}=-4 \mathrm{n} / \sigma^{3} \\
& \mathrm{~g}_{211}=\mathrm{g}_{121}=0 \\
& \mathrm{~g}_{212}=\mathrm{g}_{122}=0
\end{aligned}
$$

Os simbolos de Christoffel são dados por

$$
\begin{aligned}
& \Gamma_{\mathrm{ijk}}=1 / 2\left(E_{\mathrm{i}} \mathrm{g}_{\mathrm{jk}}+\mathrm{E}_{\mathrm{j}} \mathrm{g}_{\mathrm{ik}}-\mathrm{E}_{\mathrm{k}} \mathrm{g}_{\mathrm{ij}}\right) \\
& \Gamma_{111}=1 / 2\left(E_{1} g_{11}+E_{1} g_{11}-E_{1} g_{11}\right)=1 / 2(2.0+0)=0 \\
& \Gamma_{112}=1 / 2\left(E_{1} g_{12}+E_{1} g_{12}-E_{2} g_{11}\right)=1 / 2\left(0+2 n / \sigma^{3}+0\right)=n / \sigma^{3} \\
& \Gamma_{121}=\Gamma_{211}=1 / 2\left(E_{1} g_{21}+E_{2} g_{11}-E_{12} g_{12}\right)=1 / 2\left(2.0-2 n / \sigma^{3}\right)=n / \sigma^{3} \\
& \Gamma_{122}=\Gamma_{212}=1 / 2\left(E_{1} g_{22}+E_{2} g_{12}-E_{2} g_{12}\right)=1 / 2(0+0+0)=0 \\
& \Gamma_{221}=1 / 2\left(E_{2} g_{21}+E_{2} g_{21}-E_{1} g_{22}\right)=1 / 2(0+0+0)=0 \\
& \Gamma_{\mathrm{ij}}^{\mathrm{k}}=\mathrm{g}^{\mathrm{kl}} \Gamma_{\mathrm{ijl}} \\
& \Gamma_{11}^{1}=g^{11} \Gamma_{111}+g^{12} \Gamma_{112}=0+0=0 \\
& \Gamma_{11}^{2}=\mathrm{g}^{21} \Gamma_{111}+\mathrm{g}^{22} \Gamma_{112}=0+1 / 2 \sigma=1 / 2 \sigma \\
& \Gamma_{12}^{2}=\Gamma_{21}^{2}=\mathrm{g}^{21} \Gamma_{121}+\mathrm{g}^{22} \Gamma_{122}=0+0=0 \\
& \Gamma_{12}^{1}=\Gamma_{21}^{1}=\mathrm{g}^{11} \Gamma_{121}+\mathrm{g}^{12} \Gamma_{122}=-1 / \sigma+0=-1 / \sigma \\
& \Gamma_{22}^{2}=\mathrm{g}^{21} \Gamma_{221}+\mathrm{g}^{22} \Gamma_{222}=0+0=0
\end{aligned}
$$

\section{2)Distribuição Gama} dada por

Neste caso, a matriz de informação de Fisher, considerando apenas uma observação, é

$$
\begin{aligned}
& g_{11}=\beta / \mu^{2} \\
& g_{12}=g_{21}=0 \\
& g_{22}=\phi(\beta)
\end{aligned}
$$

Os elementos da inversa de Fisher são dados por 
$\operatorname{det} g=\beta \phi(\beta) / \mu^{2}$

$\mathrm{g}^{11}=\mathrm{g}_{22} / \operatorname{det} \mathrm{g}=\mu^{2} / \beta$

$\mathrm{g}^{22}=\mathrm{g}_{11} /$ det $\mathrm{g}=1 / \phi(\beta)$

$\mathrm{g}^{12}=\mathrm{g}^{12}=-\mathrm{g}_{12} / \operatorname{det} \mathrm{g}=0$

As derivadas de $g_{\mathrm{ij}}$ são dadas por:

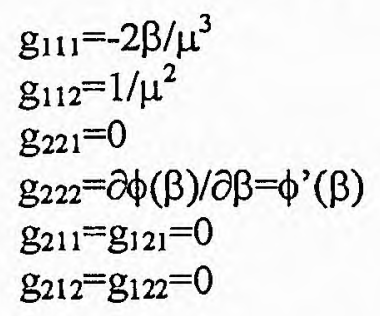

Os simbolos de Christoffel (ver Apêndice 1) são dados por,

$\Gamma_{\mathrm{ijk}}=1 / 2\left(\mathrm{E}_{\mathrm{i}} \mathrm{g}_{\mathrm{jk}}+\mathrm{E}_{\mathrm{j}} \mathrm{g}_{\mathrm{ik}}-\mathrm{E}_{\mathrm{k}} \mathrm{g}_{\mathrm{ij}}\right)$

$\Gamma_{111}=1 / 2\left(E_{1} g_{11}+E_{1} g_{11}-E_{1} g_{11}\right)=-\beta / \mu^{3}$

$\Gamma_{112}=1 / 2\left(E_{12} g_{12}+E_{1} g_{12}-E_{2} g_{11}\right)=-1 /\left(2 \mu^{2}\right)$

$\Gamma_{121}=\Gamma_{211}=1 / 2\left(E_{1} g_{21}+E_{2} g_{11}-E_{1} g_{12}\right)=1 / 2\left(2.0-2 n / \sigma^{3}\right)=1 / 2 \phi^{\prime}(\beta)$

$\Gamma_{122}=\Gamma_{212}=1 / 2\left(E_{1} g_{22}+E_{2} g_{12}-E_{2} g_{12}\right)=0$

$\Gamma_{221}=1 / 2\left(E_{2} g_{21}+E_{2} g_{21}-E_{1} g_{22}\right)=0$

$\Gamma_{\mathrm{ij}}^{\mathrm{k}}=\mathrm{g}^{\mathrm{kl}} \Gamma_{\mathrm{j} \mathrm{j} 1}$

$\Gamma_{11}^{1}=\mathrm{g}^{11} \Gamma_{111}+\mathrm{g}^{12} \Gamma_{112}=-1 / \mu$

$\Gamma_{11}^{2}=\mathrm{g}^{21} \Gamma_{111}+\mathrm{g}^{22} \Gamma_{112}=-1 /\left(2 \mu^{2} \phi(\beta)\right)$

$\Gamma_{12}^{2}=\Gamma^{2}{ }_{21}=\mathrm{g}^{21} \Gamma_{121}+\mathrm{g}^{22} \Gamma_{122}=0$

$\Gamma_{12}^{1}=\Gamma^{1}{ }_{21}=\mathrm{g}^{11} \Gamma_{121}+\mathrm{g}^{12} \Gamma_{122}=1 /(2 \beta)$

$\Gamma_{22}^{2}=\mathrm{g}^{21} \Gamma_{221}+\mathrm{g}^{22} \Gamma_{222}=1 / 2 \phi^{\prime}(\beta) / \phi(\beta)$

O tensor de Riemann (ver Apêndice 1) é dado por,

$R_{1212}=-\left[\phi(\beta)+\beta \phi^{\prime}(\beta)\right] /\left[4 \mu^{2} \beta \phi(\beta)\right]$,

e a curvatura seccional é dada por

$\mathrm{K}=-\mathrm{R}_{1212} \mathrm{~g}^{11} \mathrm{~g}^{22}=1 / 4\left[\phi(\beta)+\beta \phi^{\prime}(\beta)\right] /\left[\beta^{2} \phi(\beta)\right]$

Fig 2.1.Modelo Normal e Gama para $x=1$
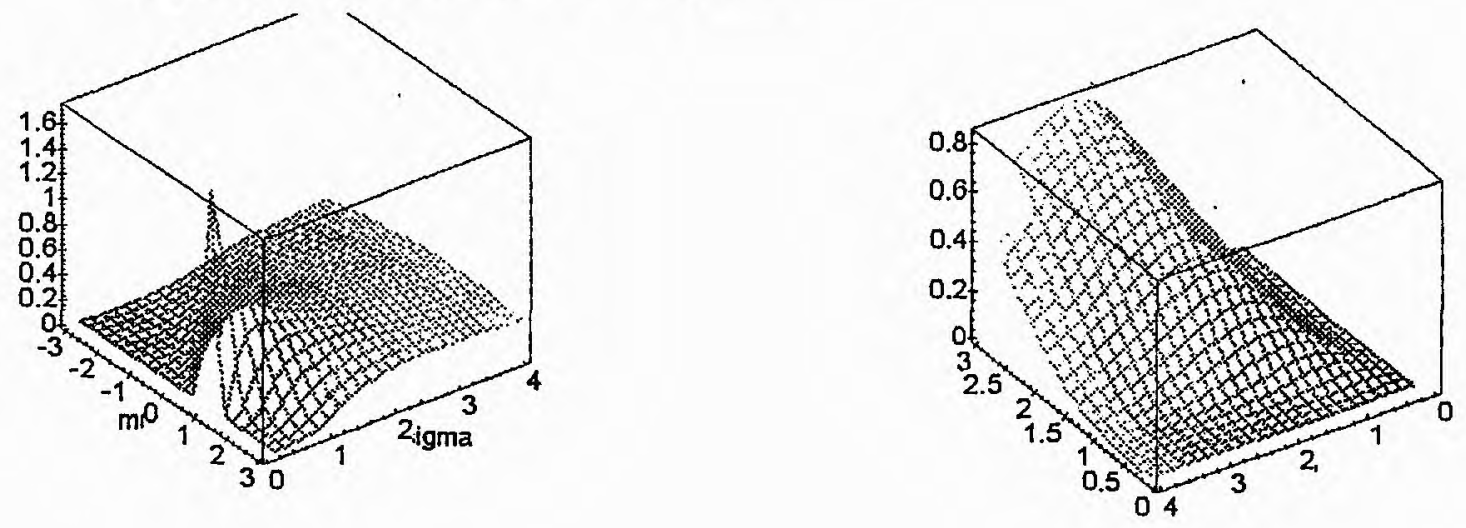
Geometrias Observadas

Para espaços paramétricos de dimensão dois, é possível provar que (ver apêndice)

$\mathrm{K}=-1 / 4 \operatorname{det}(\mathrm{M}) / \operatorname{det}(\mathrm{G})$

O significado das matrizes $\mathrm{M}$ e $\mathrm{G}$ estão no apêndice.

Como consequência do teorema Egregium de Gauss, $\mathrm{K}$ é invariante sob reparametrizações. Isso ocorre se utilizarmos $\mathrm{G}$ como a matriz de Fisher esperada, cujos termos são produtos

internos entre os versores que são a base vetorial do espaço tangente.

No entanto, se usarmos como $\mathrm{G}$ a matriz de Fisher observada, como uma aproximação da esperada, $\mathrm{K}$ varia com a reparametrização. $\mathrm{K}$ torna-se uma medida de curvatura que distingue uma mesma distribuição sob parametrizações diferentes. É dessa forma que calcularemos $\mathrm{K}$ ao longo dos exemplos do restante do trabalho. Essa metodologia foi proposta por Barndorff-Nielsen (1986) e se refere a geometrias observadas por se utilizar da matriz de Fisher observada. 


\section{Capítulo 3}

\section{Algumas aplicações numéricas}

\subsection{Conexão entre aproximação de Laplace e tensores associados}

\section{Distribuição Binomial}

Se a verossimilhança é parametrizada em termos da probabilidade de sucesso $\theta$, numa distribuição binomial, temos:

$L(\theta \mid x)=\theta^{x}(1-\theta)^{n-x}$

Considerar uma priori de Jeffreys (ver por exemplo, Box e Tiao, 1973) dada por, $\pi(\theta) \alpha \theta^{-1 / 2}(1-\theta)^{-1 / 2}$

A média exata a posteriori para $\theta$ é dada por

$$
E(\theta \mid x)=(x+1 / 2) /(n+1)
$$
dada por

Uma aproximação de Laplace para a média a p̉osteriori na parametrização original é

$$
(n-1)^{n+1 / 2}(x+1 / 2)^{x+1} /\left[n^{n+3 / 2}(x-1 / 2)^{x}\right]
$$

Uma possível reparametrização para $\theta$ é dada por $\operatorname{arcsen}\left(\theta^{1 / 2}\right)$. A função de verossimilhança nesta reparametrização é dada por

$$
L(\phi \mid x)=\left(\operatorname{sen}^{2}(\phi)\right)^{x}\left(\cos ^{2}(\phi)\right)^{n-x}
$$

Neste caso a priori de Jeffreys é dada por $\pi(\phi) \alpha$ constante, assim a aproximação de Laplace para a média a posteriori é dada por:

$$
\mathrm{n}^{\mathrm{n}+1 / 2}(\mathrm{x}+1)^{\mathrm{x}+1} /\left[(\mathrm{n}+1)^{\mathrm{n}+3 / 2} \mathrm{x}^{\mathrm{x}}\right]
$$

$\mathrm{Na}$ Figura 3.1, tem-se um gráfico dos erros em porcentagem de $E(\theta \mid x)$ versus $\mathrm{x}$ na parametrização $\theta \mathrm{com} n=18$ e na figura 3.2 tem-se um gráfico dos erros em porcentagem de $\mathrm{E}(\theta \mid \mathrm{x})$ na parametrização $\phi$ para $\mathrm{n}=18$. 


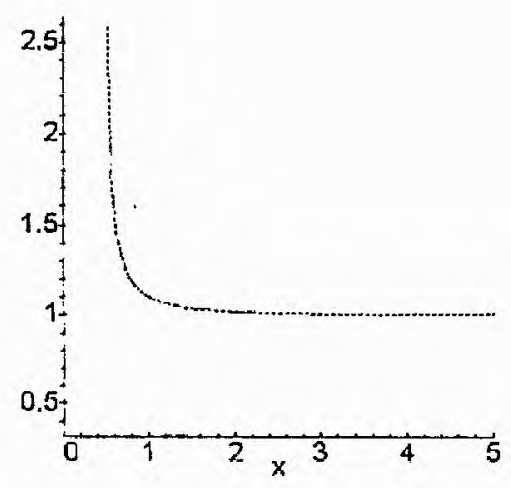

Figura 3.1: Erros percentuais de $\mathrm{E}(\theta \mid \mathrm{x})$ para a parametrização $\theta$

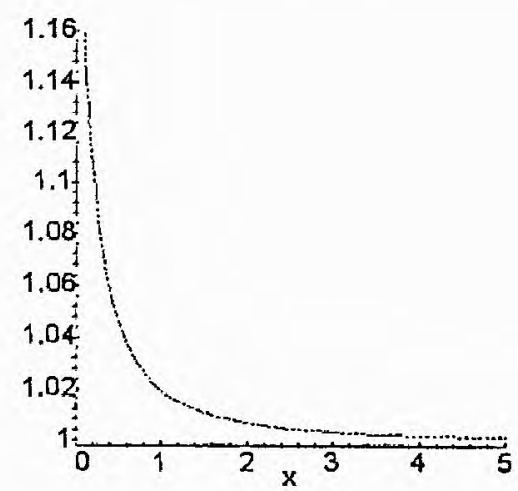

Figura 3.2:Erros percentuais de $E(\theta \mid x)$ para a reparametrização $\phi$

Notamos que para $n=18$. a parametrização $\phi$ é mais satisfatória pois os erros percentuais são menores nesta parametrização.

A seguir, calculamos os símbolos de Cristofell de segunda espécie. Como temos uma distribuição uniparamétrica, a imergimos em uma bivariada, onde a segunda distribuição tem parâmetro distinto da binomial.

O símbolo de Cristofell de interesse é dado por:

$(\mathrm{ijk})=\Gamma_{\mathrm{ij}}^{\mathrm{k}}=\mathrm{g}^{\mathrm{kl}} \Gamma_{\mathrm{ijl}}$

$\Gamma_{\mathrm{ijk}}=1 / 2\left(\mathrm{E}_{\mathrm{i}}\left(\mathrm{g}_{\mathrm{jk}}\right)+\mathrm{E}_{\mathrm{j}}\left(\mathrm{g}_{\mathrm{ik}}\right)-\mathrm{E}_{\mathrm{k}}\left(\mathrm{g}_{\mathrm{ij}}\right)\right)$

Para as parametrizações analisadas temos:

$(111)_{\theta}=\left[-2 x+6 x \theta-6 \times \theta^{2}+1-3 \theta+3 \theta^{2}-2 \theta^{3}+2 \theta^{3} n\right] /\left[\theta(\theta-1)\left(2 x-4 x \theta-1+2 \theta-2 \theta^{2}+2 \theta^{2} n\right)\right]$

$(111)_{\phi}=\left[-\sin ^{4}(\phi)+2 \sin ^{4}(\phi) n-2 \sin ^{4}(\phi) x-2 \cos ^{4}(\phi) x+\cos ^{4}(\phi)\right] /\left[\sin (\phi) \cos (\phi)\left(2 \sin ^{2}(\phi) n-\right.\right.$ $\left.\left.2 \sin ^{2}(\phi) x-\sin ^{2}(\phi)+2 \cos ^{2}(\phi) x-\cos ^{2}(\phi)\right)\right]$

Simulação $\operatorname{com} \theta=0,3$ (ver figura 3.3) mostra $(111)_{\phi}$ complexo, mas suas componentes tanto real como imaginária se mostram menores que $(111)_{\theta}$, indicando se tratar de uma melhor reparametrização. Tal resultado se mostra coerente com os obtidos pela aproximação de Laplace. 


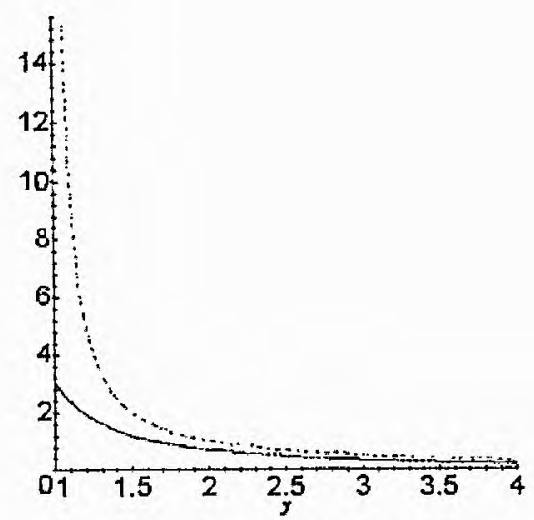

Figura 3.3: $\operatorname{Re}(111)_{\theta}$ e $\operatorname{Re}(111)_{\phi}$ onde a linha pontilhada (...) refere-se à parametrização $\theta$ e a linha continua (_ _ é associada à parametrização $\phi$

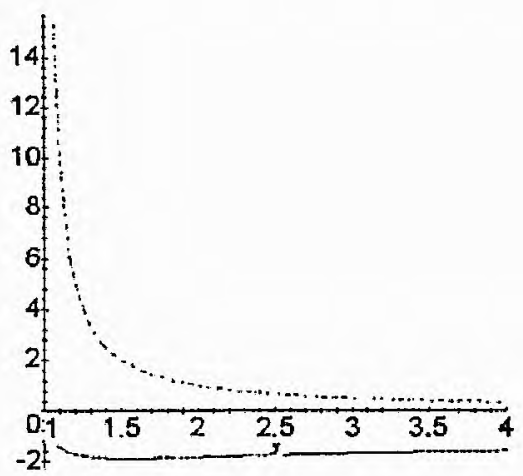

Figura 3.4: $\operatorname{Im}(111)_{\theta}$ e $\operatorname{Im}(111)_{\phi} \operatorname{com} \theta=0,3$ 
O cálculo das curvaturas geométricas usuais para o caso unidimensional, fornecem bons resultados, como observamos na figura 3.5

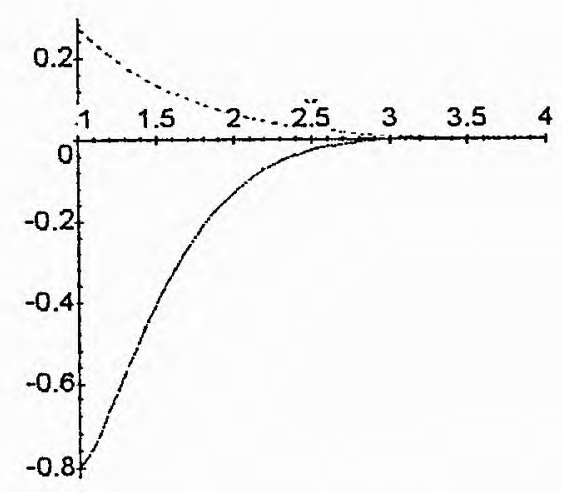

Figura 3.5. Curvaturas geométricas usuais para o caso Binomial nas parametrizações $\theta$ e $\phi$

Nas figuras $3.3,3.4,3.5$ a curva pontilhada é referente à $\theta$ e a linha contínua é referente à $\phi$. Concluímos que a parametrização $\phi$ é melhor, possuindo menores valores de curvatura.

\section{Um Exemplo bidimensional}

\subsection{Componentes de Duas Variâncias}

Considere o modelo de efeitos aleatórios $x_{j k}=\theta+e_{j}+e_{j k}, j=1, \ldots, J, k=1, \ldots, K$, onde $E\left(e_{j}\right)=E\left(e_{j k}\right)=0, \operatorname{var}\left(e_{j}\right)=\sigma^{2}{ }_{2}$ e $\operatorname{var}\left(e_{j k}\right)=\sigma^{2}{ }_{1}$. Assumir uma priori não informativa para $\theta, \sigma^{2}{ }_{1} e$ $\sigma^{2}$ dada por

$\pi\left(\theta, \sigma_{1}^{2}, \sigma_{2}^{2}\right) \propto \sigma^{-2}\left(\sigma^{2}{ }_{1}+K \sigma_{2}^{2}\right)^{-1}$

(Ver, por exemplo, Box e Tiao(1973)). A densidade a posteriori é dada por

$\pi\left(\theta, \sigma_{1}^{2}, \sigma^{2}{ }_{2}\right) \alpha\left(\sigma^{2}\right)^{-(\mathrm{v} 1 / 2+1)}\left(\sigma_{1}^{2}+\mathrm{K} \sigma^{2}{ }_{2}\right)^{-(\mathrm{v} 2 / 2+1)} \exp \left[-1 / 2\left(\mathrm{v}_{1} \mathrm{~m}_{1} / \sigma^{2}{ }_{1}+\mathrm{v}_{2} \mathrm{~m}_{2} /\left(\sigma^{2}{ }_{1}+\mathrm{K} \sigma^{2}{ }_{2}\right)\right)\right]$

onde $\mathrm{v}_{1}=\mathrm{J}(\mathrm{K}-1), \mathrm{v}_{2}=\mathrm{J}-1, \mathrm{v}_{1} \mathrm{~m}_{1}=\mathrm{S}_{1}, \mathrm{v}_{2} \mathrm{~m}_{2}=\mathrm{S}_{2}, \mathrm{~S}_{1}=\Sigma_{\mathrm{j}} \Sigma_{\mathrm{k}}\left(\mathrm{x}_{\mathrm{jk}}-\mathrm{x}_{\mathrm{j}}\right)^{2}, \mathrm{~S}_{2}=\mathrm{K} \Sigma_{\mathrm{j}}\left(\mathrm{x}_{\mathrm{j} .}-\mathrm{x}_{\mathrm{f}}\right)^{2}$.

Em Achcar e Smith (1990), temos uma comparação dos erros nas aproximações de Laplace para várias funções de interesse: 


\begin{tabular}{|l|l|l|l|}
\hline & Exata & $\left(\sigma_{1}^{2}, \sigma_{2}^{2}\right)$ & $\begin{array}{l}\left(\ln \left(\sigma^{2}{ }_{1}\right), \mathrm{K}^{-1}\right. \\
\left.\ln \left(\sigma^{2}{ }_{1}+\mathrm{K} \sigma^{2}\right)\right)\end{array}$ \\
\hline $\mathrm{E}\left(\sigma_{12}^{2} / \sigma_{1}^{2} \mid \mathrm{x}\right)$ & 0 & 19,80 & 2,53 \\
\hline $\mathrm{E}\left(\sigma_{12}^{2} \mid \mathrm{x}\right)$ & 0 & 20,59 & 2,54 \\
\hline $\mathrm{E}\left(\sigma_{1}^{2} \mid \mathrm{x}\right)$ & 0 & 0,27 & 0,27 \\
\hline $\mathrm{E}\left(\sigma_{2}^{2}{ }_{\mathrm{x}}\right)$ & 0 & 510,73 & 0,20 \\
\hline
\end{tabular}

Tabela 3.1.Erros nas aproximações de Laplace para caso de Duas Variâncias

$\left(\mathrm{K}=5 ; \mathrm{S}_{1}=58830,00 ; \mathrm{v}_{1}=24 ; \mathrm{m}_{1}=2451,25 ; \mathrm{S}_{2}=56357,00 ; \mathrm{v}_{2}=5 ; \mathrm{m}_{2}=11271,50\right)$

Conclui-se que a reparametrização considerada reduz de forma acentuada o erro.

Neste caso, os tensores são dados por:

a)Parametrização canônica $\left(\sigma_{1}^{2}, \sigma_{2}^{2}\right)$ :

$\mathrm{R}_{1212}=0,343526698910^{-25}$

b)Reparametrização $\left(\ln \left(\sigma^{2}{ }_{1}\right), \mathrm{K}^{-1} \ln \left(\sigma^{2}{ }_{1}+\mathrm{K} \sigma^{2}{ }_{2}\right)\right)$

$\mathrm{R}_{1212}=0$

\begin{tabular}{|l|l|l|}
\hline & $\left(\sigma_{1}^{2} \sigma^{2}{ }_{2}\right)$ & $\left(\ln \left(\sigma^{2}{ }_{1}\right), \mathrm{K}^{-1} \ln \left(\sigma^{2}{ }_{1}+\mathrm{K \sigma}_{2}^{2}\right)\right)$ \\
\hline $\mathrm{K}$ & $.139030405910^{-7}$ & 0 \\
\hline
\end{tabular}

Tabela 3.2. escalar de Ricci para o caso de componentes de duas Variâncias

Um fato notável ocorre: as expressões matemáticas dos símbolos de Christofell da reparametrização são muito simplificados e não dependem dos parâmetros; podemos supor que esse fato seja o principal responsável pela alta precisão obtida pela melhor reparametrização.

A tabela 3.2 mostra os valores do escalar de Ricci nas duas parametrizações. Mais uma vez o menor escalar de Ricci se apresenta na melhor parametrização dada por $\left(\ln \left(\sigma^{2}{ }_{1}\right), \mathrm{K}^{-1}\right.$ $\left.\ln \left(\sigma_{1}^{2}+\mathrm{K} \sigma^{2}{ }_{2}\right)\right)$

\subsection{Conexão entre o tensor de Riemann e dados censurados \\ Distribuição Weibull e Gumbel}

Considerar o conjunto de dados de sobrevivência da tabela 3:

$[39,4 ; 45,3 ; 49,2 ; 49,4 ; 51,3 ; 52,0 ; 53,2 ; 53,2 ; 54,9 ; 55,5 ; 57,1 ; 57,2 ; 57,5 ; 59,2 ; 61,0 ; 62,4 ; 63,8 ;$ $64,3 ; 67,3 ; 67,7]$

Tabela 3.3. Dados de sobrevivência de $n=20$, cabos elétricos submetidos a um teste industrial (valores de stress em $\mathrm{KV} / \mathrm{mm}$ )

Para os dados da tabela 3.3, ajustamos uma distribuição Weibull com dois parâmetros. O logaritmo da função de verossimilhança é dado por,

$$
1(t, \alpha, \beta)=r \ln (\beta)-r \beta \ln (\alpha)+(\beta-1) \sum_{i \varepsilon D} \ln \left(t_{i}\right)-\Sigma^{n}{ }_{i=1}\left(t_{i} / \alpha\right)^{\beta}
$$

onde n é o tamanho amostral, r é o número de falhas observadas e D é o conjunto com falhas observadas 
Os dados foram censurados (censura tipo 1) sucessivamente a começar do último. Calculamos os estimadores de máxima verossimilhança para os parâmetros da distribuição Weibull por Newton-Rhapson. Os resultados estão na tabela 3.4.

\begin{tabular}{|l|l|l|}
\hline Número de censuras & $\beta^{+}$ & $\alpha^{+}$ \\
\hline 0 & 9,1410 & 59,1245 \\
\hline 1 & 8,6368 & 59,3190 \\
\hline 2 & 8,1393 & 59,5754 \\
\hline 3 & 7,7378 & 59,9238 \\
\hline 4 & 7,3393 & 60,3533 \\
\hline 5 & 6,9741 & 60,8825 \\
\hline 6 & 6,6402 & 61,5246 \\
\hline 7 & 6,3510 & 62,2909 \\
\hline 8 & 6,1030 & 63,1917 \\
\hline 9 & 5,8413 & 64,2769 \\
\hline 10 & 5,5544 & 65,6176 \\
\hline 11 & 5,3001 & 67,2268 \\
\hline 12 & 5,0364 & 69,2455 \\
\hline 13 & 4,8081 & 71,7057 \\
\hline 14 & 4,5295 & 75,0517 \\
\hline
\end{tabular}

Tabela 3.4.Parâmetros da Weibull ajustada aos dados da tabela (3.3)

Calculamos, a partir desses valores o tensor de Riemann e do escalar de Ricci:

\begin{tabular}{|l|l|l|}
\hline Número de censuras & $\mathrm{R}_{1212}$ & $\mathrm{~K}$ \\
\hline 0 & $-1327478336 \mathrm{e} 8$ & $-.5931666930 \mathrm{e}-9$ \\
\hline 1 & $-4018560.208 \mathrm{e} 7$ & $-.2183482878 \mathrm{e}-8$ \\
\hline 2 & $-1236043.169 \mathrm{e} 7$ & $-.8081946598 \mathrm{e}-8$ \\
\hline 3 & -472914.4965 & $-.2428765857 \mathrm{e}-7$ \\
\hline 4 & -182382.3142 & $-.7446953960 \mathrm{e}-7$ \\
\hline 5 & -75967.57233 & $-.2173875275 \mathrm{e}-6$ \\
\hline 6 & -34048.11220 & $-.6106008265 \mathrm{e}-6$ \\
\hline 7 & -16933.93620 & $-.1604671907 \mathrm{e}-5$ \\
\hline 8 & -9284.570148 & $-.4010053465 \mathrm{e}-5$ \\
\hline 9 & -4996.745160 & $-.1127834733 \mathrm{e}-4$ \\
\hline 10 & -2608.037973 & $-.3876128425 \mathrm{e}-4$ \\
\hline 11 & -1524.669613 & $-.1477863129 \mathrm{e}-3$ \\
\hline 12 & -967.1168818 & $-.8574037853 \mathrm{e}-3$ \\
\hline 13 & -863.5189383 & $-.1103966913 \mathrm{e}-1$ \\
\hline 14 & 2041.789140 & 4.910320893 \\
\hline
\end{tabular}

Tabela 3.5. Tensores de Riemann e escalar de Ricci para o caso de dádos com censuras na distribuição de Weibull

É usual o estatístico trabalhar com tempos na escala logaritmica. Trata-se da parametrização Gumbel ou de valor extremo:

$$
\begin{aligned}
& x=\ln (t) \\
& u=\ln (\alpha) \\
& b=1 / \beta \\
& w_{j}=\left(x_{j}-u\right) / b \\
& f\left(x_{j} ;, u, b\right)=1 / b \exp \left[\left(w_{j}\right)-\exp \left(w_{j}\right)\right]
\end{aligned}
$$


Na reparametrização Gumbel temos os seguintes valores do tensor de Riemann e do escalar de Ricci

\begin{tabular}{|l|l|l|}
\hline Número de censuras & $\mathrm{R}_{1212}$ & $\mathrm{~K}$ \\
\hline 0 & $.1022650525 \mathrm{e} 15$ & $-.7796923450 \mathrm{e}-7$ \\
\hline 1 & $.2124366788 \mathrm{e} 14$ & $-.1635238900 \mathrm{e}-6$ \\
\hline 2 & $.4420286415 \mathrm{e} 13$ & $-.3429564385 \mathrm{e}-6$ \\
\hline 3 & $.1212664106 \mathrm{e} 13$ & $-.6329923715 \mathrm{e}-6$ \\
\hline 4 & $.3300531935 \mathrm{e} 12$ & $-.1167264845 \mathrm{e}-5$ \\
\hline 5 & $.9790905860 \mathrm{e} 11$ & $-.2058407487 \mathrm{e}-5$ \\
\hline 6 & $.3151488948 \mathrm{e} 11$ & $-.3480175755 \mathrm{e}-5$ \\
\hline 7 & $.1151076671 \mathrm{e} 11$ & $-.5537005850 \mathrm{e}-5$ \\
\hline 8 & $4731173678 \mathrm{e} 10$ & $-.8338452483 \mathrm{e}-5$ \\
\hline 9 & $1828756058 \mathrm{e} 10$ & $-.1282249444 \mathrm{e}-4$ \\
\hline 10 & $636815235.8 \mathrm{e} 9$ & $-.2042961922 \mathrm{e}-4$ \\
\hline 11 & $.2601258878 \mathrm{e}-3$ & $-.1976520866 \mathrm{e}-3$ \\
\hline 12 & $86680759.28 \mathrm{e} 8$ & $-.4848218800 \mathrm{e}-4$ \\
\hline 13 & $34125372.80 \mathrm{e} 8$ & $-.7227065603 \mathrm{e}-4$ \\
\hline 14 & $10847722.89 \mathrm{e} 8$ & $-.1154189020 \mathrm{e}-3$ \\
\hline
\end{tabular}

Tabela 3.6:Tensores de Riemann e escalar de Ricci para distribuição Gumbel com o número crescente de censuras

Comparando-se os resultados das tabelas 3.5 e 3.6 , concluímos que a parametrização Gumbel apresenta menores valores de curvatura do que a parametrização Weibull.

Outro fato interessante é que a curvatura em ambos os casos aumenta com o aumento da censura, apesar desse aumento não se apresentar de forma monótona.

A medida de curvatura escalar de Ricci mostra-se bastante adequada e coerente com os resultados da aproximação de Laplace: parametrizações que levam a melhores aproximações de Laplace têm curvaturas menores.

O escalar de Ricci mostra-se sensível ao número de censuras crescentes numa amostra. Amostras com mais censuras geram curvaturas maiores. No caso específico de dados analisados que se adequaram aos modelos Weibull e a Gumbell, a Gumbell mostrou curvaturas menores. 


\subsection{Comportamento do escalar de Ricci diante da ortogonalização dos}

\section{parâmetros}

\section{Razão de Parâmetros Exponenciais}

Suponha que $x_{i j}, i=1,2, j=1, \ldots, n$ são amostras independentes de distribuições exponenciais com média $E\left(x_{i j}\right)=\theta_{i}, i=1,2$. Na parametrização $\theta=\left(\dot{\theta}_{1}, \theta_{2}\right)$ a função de verossimilhança é dada por,

$$
\mathrm{L}(\theta \mid \mathrm{x})=\left(\theta_{1} \theta_{2}\right)^{-\mathrm{n}} \exp \left(-\mathrm{nx}_{1} \cdot / \theta_{1}-\mathrm{nx}_{2} \cdot / \theta_{2}\right)
$$

onde $x_{j}=\sum^{n}{ }_{i=1} x_{j i} \quad j=1,2$ dada por,

Assumir uma priori não-informativa de Jeffreys (ver por exemplo, Box e Tiao, 1973)

$$
\pi\left(\theta_{1}, \theta_{2}\right)=1 /\left(\theta_{1} \theta_{2}\right)
$$

Supor como parâmetro de interesse a razão $\rho=\theta_{1} / \theta_{2}$. A média a posteriori exata é dada por,

$$
\mathrm{E}(\rho / \mathrm{x})=\mathrm{n} \mathrm{x}_{1} . /(\mathrm{n}-1) \mathrm{x}_{2} .
$$

A aproximação de Laplace (ver, por exemplo, Tierney e Kadane, 1986) para $E(\rho \mid x)$ na parametrização $\left(\theta_{1}, \theta_{2}\right)$ é dada por:

$$
\left(\mathrm{x}_{1} / \mathrm{x}_{2}\right)\left[\mathrm{n}^{\mathrm{n}-3 / 2}(\mathrm{n}+2)^{\mathrm{n}+1 / 2} /(\mathrm{n}+1)^{2 \mathrm{n}-1}\right]
$$

Uma parametrização natural é dada por $\theta_{1}$ e $\rho=\theta_{1} / \theta_{2}$.

Considerando uma priori de Jeffreys proporcional à $1 /\left(\theta_{1} \rho\right)$, a aproximação de Laplace para $E(\rho \mid x)$ é dada por:

$$
\left(x_{1} / x_{2} .\right)\left[n^{n+1 / 2}(n+1)^{n-3 / 2} /(n-1)^{n-1 / 2}(n+2)^{n-1 / 2}\right]
$$

Outra parametrização é dada por parâmetros ortogonais (Cox \& Reid,1987). Neste caso, dado o parâmetro de interesse $\rho$, o parâmetro ortogonal é dado por $\lambda=\theta^{2}{ }_{1} \rho^{-1}$. A priori é proporcional à $1 /(\rho \lambda)$ e a aproximação de Laplace para $E(\rho \mid x)$ é dada por,

$$
\left(\mathrm{x}_{1} / \mathrm{x}_{2}\right)\left[(\mathrm{n}+1)^{2 \mathrm{n}-1} / \mathrm{n}^{\mathrm{n}-1 / 2}(\mathrm{n}+2)^{\mathrm{n}-1 / 2}\right]
$$

Na tabela (3.7) temos uma ilustração numérica com $n=4$ (dados de Hills, 1987). Observa-se que a melhor aproximação de Laplace é dada na parametrização com uma priori implicita localmente uniforme para os parâmetros.

\begin{tabular}{|l|l|l|l|}
\hline Exata & Aprox de Laplace & Erros(\%) & $\mathrm{K}$ \\
\hline$\left(\theta_{1}, \theta_{2}\right)$ & 1,6450 & 0 & \\
\hline$\left(\theta_{1}, \rho\right)$ & 1,4272 & 15,26 & 2.403554372 \\
\hline$(\lambda, \rho)$ & 1,6405 & 0,27 & -0.1286913754 \\
\hline$(\ln (\lambda), \ln (\rho))$ & 1,4233 & 15,60 & 1677.425955 \\
\hline
\end{tabular}


Tabela 3.7.Aproximações de Laplace e erros percentuais para $\mathrm{E}(\mathrm{x} . \mathrm{l} / \mathrm{x} .2)$ em várias parametrizações

Notamos que nesse caso a ortogonalização dos parâmetros levou a uma aproximação de Laplace pior. Esse fato foi captado pelo escalar de Ricci.

Um exemplo ilustrativo sobre a relação entre a curvatura de Ricci e a ortogonalização de parâmetros é dado pela Normal:

Sejam duas parametrizações da distribuição Normal univariada: $(\mu, \sigma)$ e $\left(\theta_{1}, \theta_{2}\right)$. A primeira é a parametrização usual, a segunda a de parâmetros naturais. A parametrização usual apresenta matriz esperada de Fisher diagonal, sendo $\mu$ e $\sigma$ ortogonais.

Seja $x=1$ fixo em ambas as distribuições. A tabela (3.8) mostra os valores do escalar de Ricci em dois pontos distintos:

\begin{tabular}{|l|l|l|}
\hline & $\mu=2, \sigma=3$ & $\mu=.2, \sigma=3$ \\
\hline$(\mu, \sigma)$ & 7758.524374 & 0.015550623 \\
\hline$\left(\theta_{1}, \theta_{2}\right)$ & -0.016308489 & 19905.50221 \\
\hline
\end{tabular}

Tabela 3.8: escalares de Ricci em duas parametrizações da Normal univariada para $\mathrm{x}=1$

Esse fato mostra que comparando-se uma parametrização ortogonal no sentido de Cox \& Reid (1987) com outra não ortogonal, as curvaturas de Ricci não podem distinguir entre as duas parametrizações.

\subsection{Reparametrização para distribuição de valor Extremo}

\section{Considerando T uma variável aleatória representando o tempo de uma unidade, assumimos 0 modelo}

$y=\ln (T)=\mu+\sigma W$

onde o erro $\mathrm{W}$ tem uma densidade de valor extremo $\exp \left(w-\mathrm{e}^{\mathrm{w}}\right), \mathrm{w} \in \mathrm{R}$.

Estatísticos usam resultados assintóticos para inferências sobre $\mu \mathrm{e} \sigma$. Um problema prático de grande interesse para os estatísticos é relacionado com o quão esses resultados assintóticos são satisfatórios para tamanhos amostrais pequenos ou moderados, especialmente quando $\sigma$ é pequeno $(\sigma<1)$.

Apresentamos uma reparametrização de $(\mu, \sigma)$ adequada para funções de verossimilhança de amostras pequenas ou moderadas. Encontramos transformações um-a-um $\theta_{1}(\mu, \sigma)$ e $\theta_{2}(\mu, \sigma)$ dos parâmetros $\mu$ e $\sigma$ tal que a função de verossimilhança para $\theta_{1}$ e $\theta_{2}$ esteja perto de uma densidade Normal Bivariada. Exploramos o método proposto por Anscombe (1964) e Sprott $(1973,1980)$ considerando a reparametrização $\theta_{1}$ e $\theta_{2}$ tais que as derivadas terceiras do logaritmo da função de verossimilhança para $\theta_{1}$ e $\theta_{2}$ localmente nos estimadores de máxima verossimilhança para $\theta_{1}$ e $\theta_{2}$ sejam próximos de zero para termos verossimilhanças aproximadamente Normais.

\subsubsection{Aproximação Normal para estimadores de máxima verossimilhança para $\mu$ e $\sigma$}


Sejam $T_{1}, T_{2}, \ldots, T_{n}$ os tempos de sobrevivência de uma amostra aleatória de tamanho $n$ submetidas a um experimento de confiabilidade e vamos assumir que o modelo de valor extremo é válido. Desse modo, o logaritmo da função de verossimilhança para $\mu$ e $\sigma$ é dado por:

$1(\mu, \sigma)=-n \ln \sigma+\sum_{j=1}{ }^{n}\left(w_{j}-\exp \left(w_{j}\right)\right)$

onde $w_{j}=\left(y_{j}-\mu\right) / \sigma$.

As segundas derivadas são dadas por

$$
\begin{aligned}
& 1_{\mu \mu}=-1 / \sigma^{2}\left(S_{01}\right) \\
& 1_{\mu \sigma}=1 / \sigma^{2}\left(n-S_{01}-S_{11}\right) \\
& 1_{\sigma \sigma}=1 / \sigma^{2}\left(n-2 S_{11}+2 S_{10}-S_{21}\right) \\
& \text { onde } S_{k j}=\sum_{i=1}{ }^{n}\left[\left(w_{i}\right)^{k} \exp \left(w_{i} j\right)\right] \\
& k, j=0,1,2, \ldots
\end{aligned}
$$

A matriz de Fisher para $\mu$ e $\sigma$ é dada por,

$$
\begin{aligned}
& \mathrm{g}_{11}=\mathrm{n} / \sigma^{2} \\
& \mathrm{~g}_{12}=\mathrm{g}_{21}=\mathrm{n} / \sigma^{2}(1+\Psi(1)) \\
& \mathrm{g}_{22}=\mathrm{n} / \sigma^{2}\left(1+\Psi(2)+\Psi(2)^{2}\right)
\end{aligned}
$$

onde $\Psi(\mathrm{k})$ é a função digama $\mathrm{dln} \Gamma(\mathrm{k}) / \mathrm{dk}$.

Para grandes valores de $n$, os estimadores de máxima verossimilhança $\mu^{+}$e $\sigma^{+}$têm uma distribuição normal bivariada $\mathrm{N}\left\{(\mu, \sigma) ; \mathrm{I}^{-1}\left(\mu^{+}, \sigma^{+}\right)\right\}$. Na prática, é usual considerar a matriz de informação observada $I_{0}$ consistindo das segundas derivadas de $l(\mu, \sigma)$ localmente em $\mu$ e $\sigma$ no lugar da matriz de informação de Fisher.

Para essa distribuição os símbolos de Christoffel de segunda espécie são dados por

$$
\begin{aligned}
& (111)=[1-\gamma] /[\sigma(-2+\gamma)] \\
& (112)=\phi /[\sigma(-2+\gamma)] \\
& (121)=\phi /[\sigma(-2+\gamma)] \\
& (122)=\phi[1-\gamma] /[\sigma(-2+\gamma)] \\
& (211)=-1 /[\sigma(-2+\gamma)] \\
& (212)=-[1-\gamma] /[\sigma(-2+\gamma)] \\
& (221)=-[1-\gamma] /[\sigma(-2+\gamma)] \\
& (222)=-2[1-\gamma]^{2} /[\sigma(-2+\gamma)]+\phi /[\sigma(-2+\gamma)] \\
& \text { onde } \\
& \phi=3-3 \gamma+\gamma^{2}
\end{aligned}
$$

O tensor de Riemann é dado por

$R_{1212}=-n / \mu^{2}$

O escalar de Ricci é dado por 


\section{$\mathrm{K}=1.405694420 / \mathrm{n}$}

Notamos que para pequenos valores de n, o escalar de Ricci tem valores maiores, caindo assintoticamente a zero.

De $\mathrm{I}_{\mu}=0$ e $\mathrm{l}_{\sigma}=0$, temos $\mathrm{S}_{01}{ }^{+}=\mathrm{n}$ e $\mathrm{S}_{11}{ }^{+}=\mathrm{n}+\mathrm{S}_{10}{ }^{+}$, onde $\mathrm{w}_{\mathrm{i}}{ }^{+}=\left(\mathrm{y}_{\mathrm{i}}-\mu^{+}\right) / \sigma^{+}$e $\mathrm{S}_{\mathrm{kj}}{ }^{+}$é o valor de $S_{\mathrm{kj}}$ calculado no estimador de máxima verossimilhança. Localmente em $\mu^{+}$e $\sigma^{+}$, temos:

$$
\begin{aligned}
& 1_{\mu \mu \mu}=n / \sigma \\
& 1_{\mu \mu \sigma}=1 / \sigma^{3}\left\{3 n+S_{10}{ }^{+}\right\} \\
& 1_{\sigma \sigma \sigma}=1 / \sigma^{3}\left\{4 n+6 S_{21}{ }^{+}+S_{31}{ }^{+}\right\} \\
& 1_{\mu \sigma \sigma}=1 / \sigma^{3}\left\{4 n+4 S_{10}{ }^{+}+S_{21}{ }^{+}\right\}
\end{aligned}
$$

Tais valores são grandes, especialmente para pequenos valores de $\sigma$.

Esses fatos implicam que a aproximação Normal para função de verossimilhança para $\mu$ e $\sigma$ pode ser bastante pobre para amostras de tamanho pequeno e moderado.

\subsubsection{Uma transformação útil dos parâmetros}

Procuramos por uma transformação um-a-um $\theta_{1}(\mu, \sigma)$ e $\theta_{2}(\mu, \sigma)$ tal que a função de verossimilhança para $\theta_{1}$ e $\theta_{2}$ é aproximadamente normal. Para tal, podemos avaliar se as derivadas terceiras do logaritmo da função de verossimilhança $1\left(\theta_{1}, \theta_{2}\right)$ está próximo a zero localmente no estimador de máxima verossimilhança para $\theta_{1}$ e $\theta_{2}$ (Anscombe, 1964).

Uma outra alternativa seria procurar por uma reparametrização $\theta_{1}$ e $\theta_{2}$ de tal forma que os valores esperados das segundas derivadas do logaritmo da função de verossimilhança são constantes (Sprott, 1973,1980). No contexto Bayesiano, esta parametrização $\theta_{1}$ e $\theta_{2}$ dá uma densidade a priori de Jeffreys para $\theta_{1}$ e $\theta_{2}$ localmente uniforme, isto é,

$$
\pi\left(\theta_{1}, \theta_{2}\right)=\left\{\operatorname{det} \mathrm{I}\left(\theta_{1}, \theta_{2}\right)\right\}^{1 / 2}=\text { Constante }
$$

onde $I\left(\theta_{1}, \theta_{2}\right)$ é a matriz de informação de Fisher para $\theta_{1}$ e $\theta_{2}$.

$\operatorname{Se} \theta_{1}$ e $\theta_{2}$ é uma transformação um-a-um dos parâmetros $\mu$ e $\sigma$, tal que a densidade a priori de Jeffreys para $\theta_{1}$ e $\theta_{2}$ é localmente uniforme, verificamos que a densidade a priori para $\mu$ e $\sigma$ satisfaz,

$$
\pi(\mu, \sigma)=\text { constante }\left\{\left[\partial \theta_{1} / \partial \mu\right]\left[\partial \theta_{2} / \partial \sigma\right]-\left[\partial \theta_{1} / \partial \sigma\right]\left[\partial \theta_{2} / \partial \mu\right]\right\}
$$

Dessa forma, a priori de Jeffreys para $\mu$ e $\sigma$ é proporcional à $\sigma^{-2},-\infty<\mu<\infty$ e $\sigma>0$, verificamos que a parametrização $\theta_{1}$ e $\theta_{2}$ tal que $E\left\{-l_{\theta 1 \theta 1}\right\}=c_{1}, E\left\{-l_{\theta 2 \theta 2}\right\}=c_{2}, E\left\{-l_{\theta 1 \theta 2}\right\}=c_{3}$, onde $c_{1}, c_{2}, c_{3}$ são constantes, satisfaz a equação diferencial,

$$
\left\{\left[\partial \theta_{1} / \partial \mu\right]\left[\partial \theta_{2} / \partial \sigma\right]-\left[\partial \theta_{1} / \partial \sigma\right]\left[\partial \theta_{2} / \partial \mu\right]\right\}=\text { Constante } / \sigma^{2}
$$

Devemos pesquisar por uma transformação um-a-um $\theta_{1}$ e $\theta_{2}$ de $\mu$ e $\sigma$ entre as possiveis soluções da equação diferencial tal que os valores esperados das segundas derivadas de $1\left(\theta_{1}, \theta_{2}\right)$ são constantes ou tal que as terceiras derivadas de $1\left(\theta_{1}, \theta_{2}\right)$ localmente em $\theta_{1}{ }^{+}$e $\theta_{2}{ }^{+}$ são próximas de zero.

Tais resultados causam um escalar de Ricci na parametrização $\left(\theta_{1}, \theta_{2}\right)$ próximo a zero: 
Se os valores esperados das segundas derivadas de $1\left(\theta_{1}, \theta_{2}\right)$ são constantes, as derivadas terceiras são nulas e a matriz $\mathrm{M}$ terá segundas e terceiras linhas nulas. $\mathrm{O}$ determinante de $\mathrm{M}$ será nulo. E

$R_{1212}=-1 / 2 \operatorname{det} M / \operatorname{det} G$

Resultando em

$\mathrm{K}=\mathrm{g}^{11} \mathrm{~g}^{22} \mathrm{R}_{1212}=0$

Se considerarmos $\theta_{1}=\ln (\sigma), \theta_{2}$ será dado pela equação diferencial

$1 / \sigma \partial \theta_{2} / \partial \mu=$ constante $/ \sigma^{2}$

Sua solução é dada por,

$\theta_{2}=\int d \mu / \sigma+c(\sigma)=\mu / \sigma+c(\sigma)$

$c(\sigma)$ sendo uma função arbitrária de $\sigma$, podendo $\operatorname{ser} c(\sigma)=a / \sigma$. Daí:

$\theta_{1}=\ln (\sigma)$

$\theta_{2}=(\mu+a) / \sigma$

\subsection{Exemplo Numérico}

Na tabela 3.9 , temos os níveis de voltagem nos quais unidades falham em um experimento de laboratório:

\begin{tabular}{|l|l|l|l|l|}
\hline 32,0 & 35,4 & 36,2 & 39,8 & 41,2 \\
\hline 43,3 & 45,5 & 46,0 & 46,2 & 46,4 \\
\hline 46,5 & 46,8 & 47,3 & 47,3 & 47,6 \\
\hline 49,2 & 50,4 & 50,9 & 52,4 & 56,3 \\
\hline
\end{tabular}

Tabela 3.9: níveis de voltagem em Kilovolts/milímetro

Assumindo que o modelo Gumbel é apropriado, os estimadores de máxima verossimilhança para $\mu$ e $\sigma$ são $\mu^{+}=3,86663$ e $\sigma^{+}=0,10658$. Nesse caso $n=20, S_{10}{ }^{+}=-11,517$; $\mathrm{S}_{01}{ }^{+}=20,00 ; \mathrm{S}_{11}{ }^{+}=8,4807 ; \mathrm{S}_{21}{ }^{+}=-17,084 ; \mathrm{S}_{10}{ }^{+}=13,2369$.

Considerando-se a reparametrização $\theta_{1}=\ln (\sigma)$ e $\theta_{2}=(\mu-3,90) / \sigma$, as estimativas de máxima verossimilhança são dadas por $\theta_{1}{ }^{+}=-2,23886 \mathrm{e}$ $\theta_{2}^{+}=0$.

\begin{tabular}{|l|l|l|}
\hline Parametrização & $\mathrm{R}_{1212}$ & $\mathrm{k}$ \\
\hline$(\mu, \sigma)$ & 220106.1448 & 249878.9541 \\
\hline$\left(\theta_{1}, \theta_{2}\right)$ & 20.61205207 & 22.82734875 \\
\hline
\end{tabular}

Tabela 3.10: valores de $R_{1212}$ e k para parametrizações $(\mu, \sigma)$ e $\left(\theta_{1}, \theta_{2}\right)$

$\mathrm{Na}$ tabela 3.10 temos os valores do tensor de Riemann e da curvatura escalar de Ricci para as duas parametrizações. Notamos que a parametrização $\left(\theta_{1}, \theta_{2}\right)$ apresenta valores de curvatura muito menores que a parametrização original. 
Contornos para a função de verossimilhança para $\left(\theta_{1}, \theta_{2}\right)$ mostram-se bastante próximos da aproximação Normal, para $(\mu, \sigma)$ tais contornos estão distantes. Dessa forma, a medida de curvatura escalar de Ricci conseguiu diagnosticar qual a parametrização entre $(\mu, \sigma)$ e $\left(\theta_{1}, \theta_{2}\right)$ que apresenta melhor aproximação com a Normal.

As figuras 3.6 e 3.7 mostram os contornos da função de verossimilhança nas duas parametrizações. 


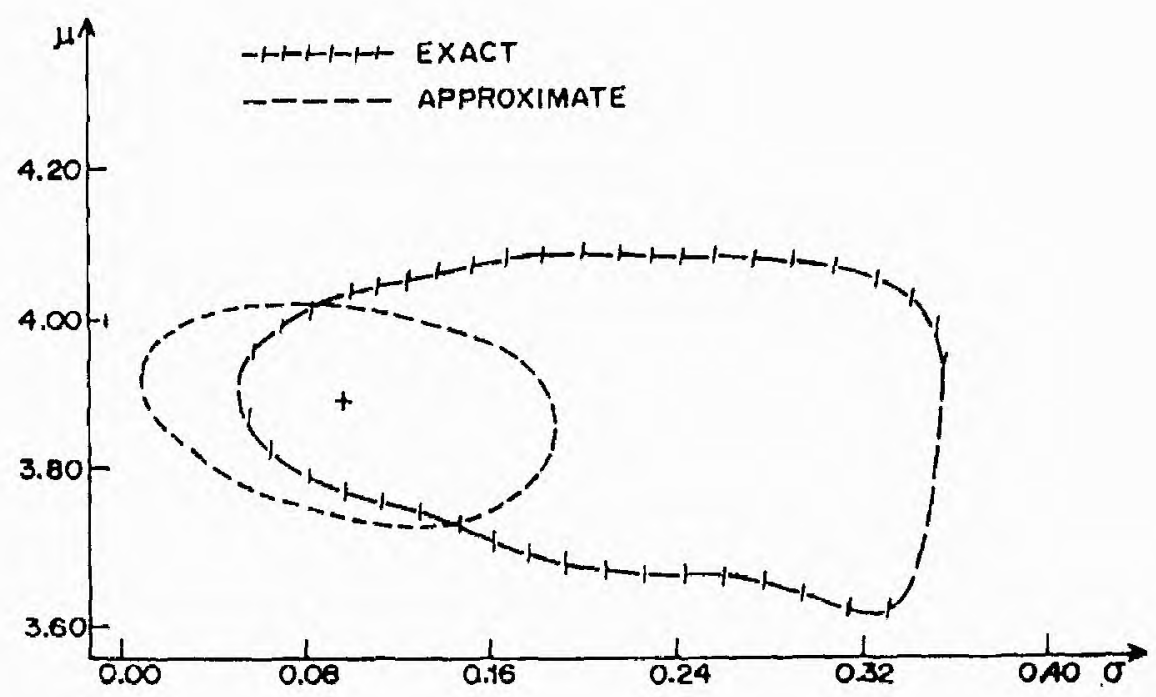

Figura 3.6. Contornos da função de verossimilhança para $\mu$ e $\sigma(1(\mu, \sigma)=-1)$

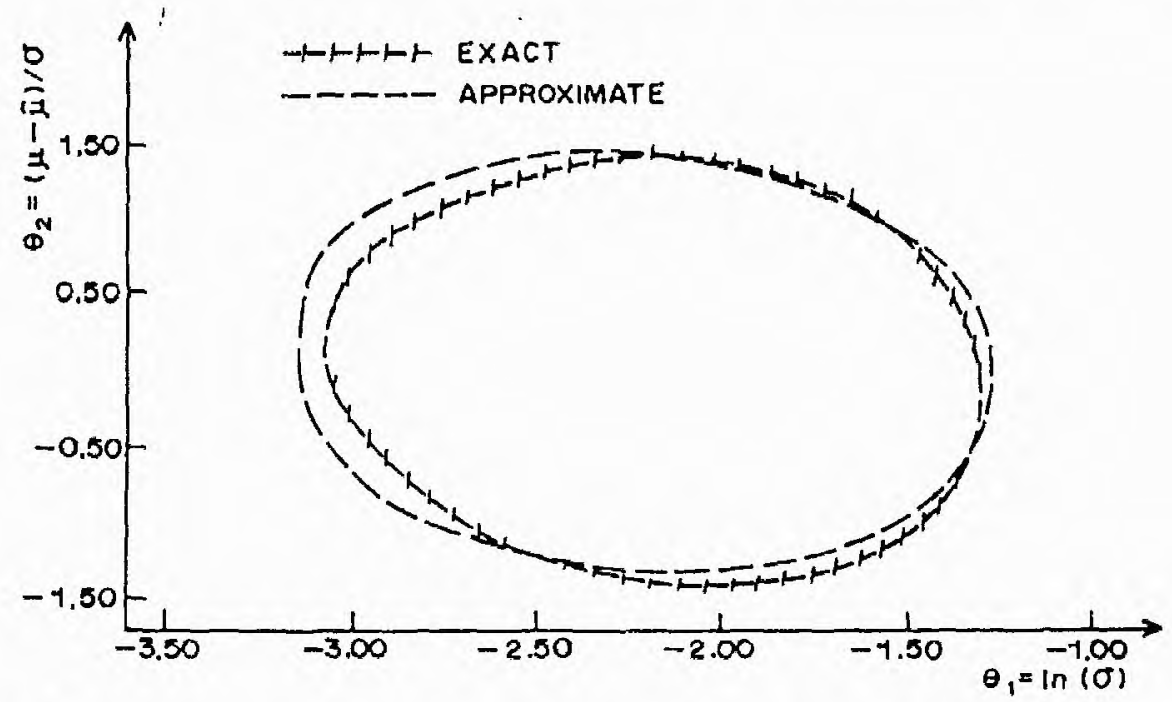

Figura 3.7. Contornos da função de verossimilhança para $\theta_{1}$ e $\theta_{2}\left(1\left(\theta_{1}, \theta_{2}\right)=-1\right)$, 


\subsubsection{Exemplo com dados censurados}

Na tabela 3.11, temos dados censurados de tipo II com as observações $y_{j}$ dadas na forma de valor extremo (logaritmos dos tempos de falhas), com $r=28$ e $n=40$ :

\begin{tabular}{|l|l|l|l|l|}
\hline$-2,982$ & $-2,849$ & $-2,546$ & $-2,350$ & $-1,983$ \\
\hline$-1,492$ & $-1,443$ & $-1,394$ & $-1,386$ & $-1,269$ \\
\hline$-1,195$ & $-1,174$ & $-0,845$ & $-0,620$ & $-0,576$ \\
\hline$-0,548$ & $-0,247$ & $-0,195$ & $-0,056$ & $-0,013$ \\
\hline 0,006 & 0,033 & 0,037 & 0,046 & 0,084 \\
\hline 0,221 & 0,245 & 0,296 & $0,296+$ & $0,296+$ \\
\hline $0,296+$ & $0,296+$ & $0,296+$ & $0,296+$ & $0,296+$ \\
\hline $0,296+$ & $0,296+$ & $0,296+$ & $0,296+$ & $0,296+$ \\
\hline
\end{tabular}
censuradas)

Tabela 3.11: dados com censura tipo II (observações com + são observações

Os estimadores de máxima verossimilhança para $\mu$ e $\sigma$ são dados por $\mu^{+}=0,1563$ e $\sigma^{+}=0,9104$. O logaritmo da função de verossimilhança para $\mu$ e $\sigma$ é dado por:

$$
1(\mu, \sigma)=-r \ln (\sigma)+\sum_{j \in D}\left(w_{j}\right)-\sum_{j=1} n \exp \left(w_{j}\right)
$$
censurados.

onde $w_{j}=\left(y_{j}-\mu\right) / \sigma$ e $D$ é o conjunto das unidades onde seus tempos de vida são

Considerando a reparametrização $\theta_{1}=\ln (\sigma)$ e $\theta_{2}=(\mu-0,16) / \sigma$, o logaritmo da função de verossimilhança para $\theta_{1}$ e $\theta_{2}$ é dado por:

$$
\begin{aligned}
& 1\left(\theta_{1}, \theta_{2}\right)=-r \ln \left(\theta_{1}\right)+\Sigma_{j \in D}\left(w_{j}\right)-\sum_{j=1}{ }^{n} \exp \left(w_{j}\right) \\
& w_{j}=\left\{y_{j}-\left[\theta_{2} \exp \left(\theta_{1}\right)+0,1563\right]\right\} / \exp \left(\theta_{1}\right) \\
& \text { e os estimadores de máxima verossimilhança: } \\
& \theta_{1}{ }^{+}=-1,855978042 \\
& \theta_{2}{ }^{+}=0
\end{aligned}
$$

Na tabela 3.12, temos os valores do tensor de Riemann e da curvatura escalar de Ricci:

\begin{tabular}{|l|l|l|}
\hline & $\mathrm{R}$ & $\mathrm{K}$ \\
\hline$\left(\theta_{1}, \theta_{2}\right)$ & 1208,233162 & 147,0213627 \\
\hline$(\mu, \sigma)$ & 1222,162378 & 148,6922927 \\
\hline
\end{tabular}

Tabela 3.12. tensor de Riemann e escalar de Ricci nas parametrizações $(\mu, \sigma)$ e $\left(\theta_{1}, \theta_{2}\right)$

Concluímos que as curvaturas na parametrização $\left(\theta_{1}, \theta_{2}\right)$ são menores que as curvaturas na parametrização $(\mu, \sigma)$.

$\mathrm{Na}$ tabela 3.13 mostramos vários intervalos de confiança para $\sigma$ :

\begin{tabular}{|l|l|}
\hline Normalidade assintótica para $\left(\mu^{+}, \sigma^{+}\right)$ & $(0,65 ; 1,17)$ \\
\hline Normal idade assintótica para $\left(\theta_{1}^{+}, \theta_{2}{ }^{+}\right)$ & $(0,69 ; 1,20)$ \\
\hline Método da Razão de Verossimilhança & $(0,70 ; 1,22)$ \\
\hline Método Exato & $(0,72 ; 1,28)$ \\
\hline
\end{tabular}

Tabela 3.13. Intervalo de Confiança $90 \%$ para $\sigma$ obtido por vários métodos 
Observamos que o intervalo de confiança obtido pela reparametrização $\left(\theta_{1}, \theta_{2}\right)$ é muito próximo do obtido pelo método da razão de verossimilhanças que é invariante por parametrizações.

\subsection{Comportamento do escalar de Ricci em uma análise Bayesiana usando Gibbs com Metrópolis-Hastings}

Assumindo-se dois tempos de falhas $\mathrm{X}$ e $\mathrm{Y}$ associados a cada unidade de observação com distribuição ACBVE de Block e Basu com função densidade conjunta, a função de verossimilhança é dada por

$L\left(\lambda_{1}, \lambda_{2}, \lambda_{3}\right)=\lambda_{1}{ }^{\mathrm{r}} \lambda^{\mathrm{n}} \lambda_{2}{ }^{\mathrm{n}-\mathrm{r}} \lambda_{23}{ }^{\mathrm{r}} \lambda_{13}{ }^{\mathrm{n}-\mathrm{r}} / \lambda_{12}{ }^{\mathrm{n}} \exp \left\{-\lambda_{1} \mathrm{x}_{\mathrm{x}}-\lambda_{2}\right.$ y. $\left.-\lambda_{3} \mathrm{Rm}\right\}$

Onde $r=\sum_{i=1}{ }^{n} \delta_{i}, R m=\sum_{i=1}{ }^{n}\left[\delta_{i} y_{i}+\left(1-\delta_{i}\right) x_{i}\right], x .=\left(\sum_{i=1}{ }^{n} x_{i}\right) / n, y=\left(\sum_{i=1}{ }^{n} y_{i}\right) / n$ e

$\delta_{\mathrm{i}}=$

a) 1 se $X_{i}<Y_{i}$,

b) 0 se $X_{i} \geq Y_{i}$.

Para inferência Bayesiana, considera-se a introdução de uma variável latente $\mathrm{N}_{1}$ $\left(\mathrm{N}_{1}=\right.$ número de observações tais que $\left.\mathrm{X}_{\mathrm{i}}<\mathrm{Y}_{\mathrm{i}}\right)$, assumindo-se as seguintes densidades a priori para: $\mathrm{N}_{1}, \lambda_{1}, \lambda_{2}$ e $\lambda_{3}$ :

$$
\begin{aligned}
& \mathrm{N} 1 \sim \mathrm{b}(\mathrm{n}, \lambda 1 / \lambda 12) \\
& \lambda 1 \sim \Gamma(\mathrm{a} 1, \mathrm{~b} 1) \\
& \lambda 2 \sim \Gamma(\mathrm{a} 2, \mathrm{~b} 2) \\
& \lambda 3 \sim \Gamma(\mathrm{a} 3, \mathrm{~b} 3)
\end{aligned}
$$

onde os $a_{i}$ e $b_{i}$ são conhecidos para $i=1,2,3$ obtidos através da interação entre o pesquisador e 0 estatístico, $b\left(n, \lambda_{1} / \lambda_{12}\right)$ denota uma distribuição binomial com probabilidade de sucesso $\lambda_{1} / \lambda_{12}, \Gamma\left(a_{i}, b_{i}\right)$ denota uma distribuição gama com média $a_{i} / b_{i}$ e variância $a_{i} / b_{i}{ }^{2}$. Assumindo-se independência a priori para $N_{1}, \lambda_{1}, \lambda_{2}, \lambda_{3}$ a distribuição conjunta a posteriori para $\mathrm{N}_{1}, \lambda_{1}, \lambda_{2}, \lambda_{3}$ é dada por:

$$
\begin{gathered}
\pi\left(N_{1}, \lambda_{1}, \lambda_{2}, \lambda_{3} \mid D\right) \alpha n ! /\left[\left(n-N_{1}\right) ! N_{1} !\right]\left(\lambda_{1} / \lambda_{12}\right)^{\mathrm{N} 1}\left(\lambda_{2} / \lambda_{12}\right)^{\mathrm{n}-\mathrm{N} 1}\left(\lambda^{\mathrm{n}} / \lambda_{12}{ }^{\mathrm{n}}\right) \lambda_{13}{ }^{\mathrm{r}} \lambda_{1}{ }^{\mathrm{r}+\mathrm{al}-1} \lambda_{2}^{\mathrm{n}-\mathrm{r}+\mathrm{a} 2-} \\
{ }^{1} \lambda_{3}{ }^{\mathrm{n} 3-1} \lambda_{23}^{\mathrm{r}} \exp \left\{-\left(\mathrm{x}+\mathrm{b}_{1}\right) \lambda_{1}-\left(\mathrm{y}+\mathrm{b}_{2}\right) \lambda_{2}-\left(\mathrm{Rm}+\mathrm{b}_{3}\right) \lambda_{3}\right\}
\end{gathered}
$$

onde $\mathrm{D}$ denota o conjunto de dados. Considerando-se as distribuições condicionais para $\lambda_{1}, \lambda_{2}, \lambda_{3}$ necessárias para aplicar a técnica do algoritmo Gibbs verifica-se que estas não possuem uma distribuição padrão, no sentido de que seja fácil gerar valores de variáveis que tenham essa distribuição. Dessa forma, usa-se o algoritmo de Metrópolis-Hastings. Para monitorar a convergência pode-se usar o critério Gelman e Rubin (1992), que usa a técnica da análise da variância para determinar a necessidade de um número maior de iterações. Usa-se uma estimativa do fator $R$, fator de escala, para se monitorar a convergência.

Distribuição ACVE em testes de vida acelerados

Considerando-se um sistema com dois componentes cujos tempos de vida são dados por $\mathrm{X}$ e $\mathrm{Y}, \mathrm{J}$ níveis de stress $\mathrm{V}_{1}, \mathrm{~V}_{2} \ldots \mathrm{V}_{\mathrm{J}}$ e assumindo-se que os tempos de vida são conduzidos com a aplicação constante de um nível de stresse selecionado, obtém-se informação sobre os tempos de vida de cada componente sob o nível de normal de stresse, $\mathrm{V}_{0}$. 
Considerando o modelo de potência dadao por

$\lambda_{\mathrm{ij}}=\mathrm{c}_{\mathrm{i}} \mathrm{V}_{\mathrm{j}}^{\mathrm{P}}$

onde $\mathrm{i}=1,2,3, \mathrm{j}=0,1,2, \ldots, \mathrm{J} ; \mathrm{c}_{1}, \mathrm{c}_{2}, \mathrm{c}_{3}$ e $\mathrm{P}$ são constantes e assumindo-se que $(X, Y)$ tem distribuição ACBVE com parâmetros $\lambda_{10}, \lambda_{20}$ e $\lambda_{30}$ sob o nível normal de stresse e que sob um nível de estresse $V_{j}, j=1,2, \ldots, J,(X, Y)$ segue o modelo ACBVE com parâmetros $\lambda_{1 j}, \lambda_{2 j}$ e $\lambda_{3 j}$ $, j=1,2 \ldots J$ e considerando-se as $n_{j}$ unidades no começo de cada teste com estresse $V_{j}$, a função de verossimilhança é dada por

$L\left(c_{1}, c_{2}, c_{3}, P\right)=c_{1}{ }^{r} c_{23}{ }^{r} c_{2}{ }^{n-r} c_{13}{ }^{n-r} c_{123}{ }^{n} c_{12}{ }^{-n} \Pi_{j=1}{ }^{j}\left(V_{j}{ }^{2 P}\right)^{n j} \exp \left\{-\left[c_{1} S_{X}(P)+c_{2} S_{Y}(P)+c_{3} T(P)\right]\right\}$

Onde $T=\sum_{j=1}{ }^{J} r_{j}, n_{=}=\sum_{j=1}{ }^{J} n_{j}, S_{X}(P)=\sum_{j=1}{ }^{J} n_{j} X_{j} V_{j}{ }^{P}, S_{Y}(P)=\sum_{j=1}{ }^{J} n_{j} Y_{j} V_{j}{ }^{P}$, $T(P)=\sum_{j=1}{ }^{J} R_{j} V_{j}{ }^{P}$.

Para a análise Bayesiana, introduz-se a variável alatente $\mathrm{N}$ representando o número de observações tais que $X_{i 1}<Y_{i 1}$ e assumem-se as seguintes densidades a priori para $N_{1}, c_{1}, c_{2}, c_{3}$ e $\mathrm{P}$ :

$$
\begin{aligned}
& N_{1} \sim b\left(n_{1}, c_{1} / c_{12}\right) \\
& c_{1} \sim \Gamma\left(a_{1}, b_{1}\right) \\
& c_{2} \sim \Gamma\left(a_{2}, b_{2}\right) \\
& c_{3} \sim \Gamma\left(a_{3}, b_{3}\right) \\
& P \sim N\left(\mu_{0}, \sigma_{0}{ }^{2}\right) \\
& a_{j}, b_{j}, \mu_{0}, \sigma_{0} j=1,2,3 \text { conhecidos }
\end{aligned}
$$

A posteriori conjunta é dada por

$$
\begin{aligned}
& \begin{array}{l}
\pi\left(N_{1}, c_{1}, c_{2}, c_{3}, P \mid D\right) \alpha n ! /\left[\left(n-N_{1}\right) ! N_{1} !\right]\left(c_{1} / c_{12}\right)^{N 1}\left(c_{2} / c_{12}\right)^{n-N 1}\left(c^{n} / c_{12}{ }^{n}\right) c_{13}{ }^{r} c_{1}{ }^{r+a 1-1} c_{2}{ }^{n-r+a 2-1} \\
c_{3}{ }^{n 3-1} c_{23}{ }^{r} \exp \left\{-\left(S_{X}(P)+b_{1}\right) c_{1}-\left(S_{Y}(P)+b_{2}\right) c_{2}-\left(T(P)+b_{3}\right) c_{3}\right\}
\end{array} \\
& \text { onde D denota o conjunto de dados }
\end{aligned}
$$


Tabela 3.14. Dados de tempo de vida bivariados gurados $\operatorname{com} \lambda_{1}=0,25, \lambda_{2}=0,16$ e $\lambda_{3}=0,0$

\begin{tabular}{rrrrrrr}
\hline$i$ & $x$ & $y$ & $i$ & $x$ & $y$ \\
\hline 1 & 3,73 & 2,54 & 16 & 3,42 & $1,0 y$ \\
2 & 5,83 & 7,74 & 17 & 7,71 & 0,33 \\
3 & 8,44 & 9,89 & 18 & 6,92 & 2,59 \\
4 & 7,95 & 2,47 & 19 & 7,76 & 3,77 \\
5 & 7,66 & 8,77 & 20 & 0,16 & 6,07 \\
6 & 3,47 & 1,86 & 21 & 7,79 & 6,98 \\
7 & 2,75 & 1,30 & 22 & 0,66 & 0,49 \\
8 & 0,57 & 5,04 & 23 & 10,83 & 4,03 \\
9 & 3,48 & 1,13 & 24 & 4,23 & 2,71 \\
10 & 4,12 & 7,24 & 25 & 3,23 & 18,74 \\
11 & 2,08 & 9,40 & 26 & 1,00 & 9,10 \\
12 & 4,19 & 1,50 & 27 & 3,08 & 12,43 \\
13 & 0,82 & 6,29 & 28 & 0,55 & 13,50 \\
14 & 1,14 & 2,61 & 29 & 0,37 & 5,52 \\
15 & 0,18 & 8,17 & 30 & 0,39 & 2,37 \\
\hline
\end{tabular}


Tabela 3.15. Dados de lompo de vida bivariados

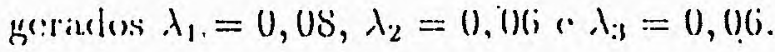

\begin{tabular}{|c|c|c|c|c|c|}
\hline$x$ & $i$ & $x$ & $j$ & $x$ & $r$ \\
\hline$(1,0115,5)$ & $19,775,12$ & $22: 141$ & |11,ind:1.4 & $17.18 \times 8$ & 0,9211 \\
\hline n.nistiot) & 9.75121 & urack: & I0,nitit & 1100 & 1,00002 \\
\hline 0,01 ixus & 48180 & 1,0019551 & 10,6182 & 1.tibitist & 11,1866 \\
\hline 0,08325 & 9,8316 & 03301 & $0,(138.1$ & 1, & 1,3900 \\
\hline 0,$11 ; 3 \cdot 2$ & 9,8570 & 07:36iti & \{1).(เiт): & 2.21413 & $1,692 \div 1$ \\
\hline $0.1: 3717$ & 9,8 & 10112 & 111.09172 & 2. & $12,0: 372$ \\
\hline $0,14.587$ & $?$ & 8 & 1 & & 2.4633 \\
\hline$(1,20) 4(18$ & $01,437,1$ & 78 & $10,7: 328$ & & 12,8087 \\
\hline $0,2: 35,78$ & (1,:15!!!) & 1,178(i)i & I11.7(ii) & & 13,1215 \\
\hline $0.28 \times 11$ & 10,0040 & rar & 1 & & 0 \\
\hline $0,3127(\mathrm{i}, 3$ & 10,0373 & 1,25401 & 10,8288 & & 167 \\
\hline $0.35206 i$ & 100580 & 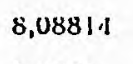 & $0,02 ! 12$ & & \\
\hline 0,37 & 10,0798 & $8,27 t ; 2$ & 23331 & & 105 \\
\hline $0,3906 \mathrm{isti}$ & 10,09 & $8,527 !)$ & 0,00153 & $(i, 15988$ & 15 \\
\hline $0,40:$ & & 1 & & (i, TOYy & 522 \\
\hline 0.11 .374 & & 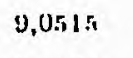 & min & $7, .5385$ & 1 \\
\hline 0,42769 & $1_{10,1219}^{10}$ & 2952 & $1,3: 311$ & .11) & 987 \\
\hline 0.1110 & & (1) & 1 , (i) (i)! & & \\
\hline u, lisisitu & 10,1519 & 0 & $1,10.9$ & & \\
\hline $0.17: 38.3$ & 10.1010 & $\operatorname{lo~}^{\circ}$ & & 10.36 & 20 \\
\hline $0,310.3+4.1$ & {$[0, J 8(i)$} & $(0,12 \cdot 1.3$ & 2,00018 & & \\
\hline 0.518 & 10100 & 15 & , & & \\
\hline $0,517: 1: 7$ & 10,2135 & 11,3216 & & & \\
\hline $0,56.35 .8$ & 10,2372 & $11,738: 3$ & $3,929 !$ & & \\
\hline $0,(111$ & & & & & $6,0,0$ \\
\hline $0,6 \cdot 1623$ & 10,3075 & 12,72 & 4,9 & 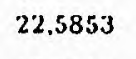 & 15,1477 \\
\hline & (4, & $20,2.30 .3$ & D, & 1.3 .382 .9 & $22,098.3$ \\
\hline 70017 & 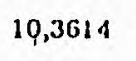 & $0_{0}$ & 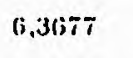 & 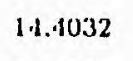 & 0 \\
\hline & 3955 & & & 15. & \\
\hline 0,81160 & 20,8100 & & 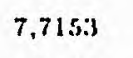 & & 31,5669 \\
\hline & 10,1842 & 15,4718 & $7,8: 3$ & .81 & \\
\hline 0,87 & $10,505 y$ & $15,8901 i$ & &. $.8306 i$ & 33 \\
\hline & 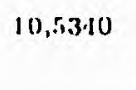 & $\begin{array}{l}\mid(i, 5) i R) \\
17,1 \text { sks }\end{array}$ & $\begin{array}{l}8,47112 \\
4, ., 8111:\end{array}$ & $2.16 \cdot 4.1$ & \\
\hline
\end{tabular}


Tabela 3.16. Dados de tempo de vida bivariados gerados com aplicação de estresse

\begin{tabular}{ccc}
\hline$V_{1}=1$ & $V_{2}=2$ & 0 \\
\hline$\left(X_{1 i}, Y_{2 i}\right)$ & $\left(X_{2 i}, Y_{2 i}\right)$ & $\left(\lambda_{3 i}, Y_{3 i}\right)$ \\
\hline$(7,65 ; 2,18)$ & $(2,29 ; 0,02)$ & $(0,34 ; 0,20)$ \\
$(16,67 ; 9,26)$ & $(0,10 ; 0,38)$ & $(950 ; 1,30)$ \\
$(39,30 ; 6,72)$ & $(0,88 ; 0,27)$ & $(0,63 ; 0,69)$ \\
$(1,30 ; 3,22)$ & $(0,45 ; 0,04)$ & $(0,68 ; 0,12)$ \\
$(9,04 ; 2,23)$ & $(1,66 ; 1,60)$ & $(3,22 ; 0,09)$ \\
$(5,15 ; 0,41)$ & $(0,74 ; 1,67)$ & $(1,91 ; 0,91)$ \\
$(5,20 ; 5,91)$ & $(2,50 ; 0,37)$ & $(0,52 ; 0,58)$ \\
$(5,00 ; 0,84)$ & $(3,50 ; 0,03)$ & $(0,30 ; 0,01)$ \\
$(5,66 ; 0,42)$ & $(8,45 ; 0,71)$ & $(1,30 ; 0,02)$ \\
$(11,80 ; 0,15)$ & $(4,60 ; 0,83)$ & $(0,52 ; 0,10)$ \\
$(17,08 ; 10,37)$ & $(2,66 ; 1,06)$ & $(2,08 ; 0,30)$ \\
$(17,92 ; 0,76)$ & $(1,16 ; 1,04)$ & $(0,95 ; 0,91)$ \\
$(1,62 ; 2,73)$ & $(1,03 ; 0,41)$ & $(0,43 ; 0,02)$ \\
$(1,42 ; 1,85)$ & $(4,36 ; 1,34)$ & $(0,25 ; 0,08)$ \\
$(3,60 ; 1,50)$ & $(0,76 ; 0,77)$ & $(1,39 ; 0,08)$
\end{tabular}


Análise do conjunto de dados I

Na tabela 3.14 temos observações bivariadas $(X, Y)$ geradas, considerando-se 0 modelo ACBVE com parâmetros $\lambda_{1}=0.25, \lambda_{2}=0.16$ e $\lambda_{3}=0$.

Para a inferência Bayesiana assumem-se as densidades a priori $\mathrm{N}_{1} \sim \mathrm{b}\left(30, \lambda_{1} / \lambda_{12}\right)$, $\lambda_{1} \sim \Gamma\left(a_{1}, b_{1}\right), \lambda_{2} \sim \Gamma\left(a_{2}, b_{2}\right), \lambda_{3} \sim \Gamma\left(a_{3}, b_{3}\right)$, onde $a_{1}=115, b_{1}=480, b_{2}=378, a_{2}=56, a_{3}=20, b_{3}=1400$, $r=16, n-r=14, n=30, x=114.51, y=165.67, R m=207.77$. Leandro (1997) obteve os seguintes resultados:

10 cadeias de tamanho 2000

utilizando-se 10 pontos iniciais para $\mathrm{N}_{1}, \lambda_{1}, \lambda_{2}, \lambda_{3}$

desprezando-se 1000 primeiras iterações

tomando os pontos de $10 \mathrm{em} 10$

\begin{tabular}{|l|l|l|l|}
\hline & $\lambda_{1}$ & $\lambda_{2}$ & $\lambda_{3}$ \\
\hline EMV & 0.2485 & 0.1698 & 0.0164 \\
\hline Confiança 95\% & $(0.07010 ; 0.4260)$ & $(0.0090,0.3310)$ & $(-0.1830,0.2160)$ \\
\hline $\begin{array}{l}\text { Cred 95\%[ Priori } \\
\text { não informativa] }\end{array}$ & $(0.700,0.4360)$ & $(0.0500,0.3360)$ & $(-0.1820,0.2100)$ \\
\hline $\begin{array}{l}\text { Cred 95\%[Priori } \\
\text { informativa] }\end{array}$ & $(0.2120,0.2920)$ & $(0.1330,0.1990)$ & $(0.0060,0.0440)$ \\
\hline Cred 95\%[G-MH] & $(0.2051,0.2942)$ & $(0.1342,0.2010)$ & $(0.0098,0.0249)$ \\
\hline
\end{tabular}

Tabela 3.14. resultados de inferência dos parâmetros do conjunto de dados I

Os escalares de Ricci são dados por:

Caso Clássico:

$\mathrm{K}=-0.0005931030489$

$\mathrm{K}\left(\lambda_{\mathrm{l}}\right)=-5.895673301 \mathrm{e}-3$

$\mathrm{K}\left(\lambda_{2}\right)=-5.936546945 \mathrm{e}-3$

$\mathrm{K}\left(\lambda_{3}\right)=-4.455205390 \mathrm{e}-3$

Caso Bayesiano com priori informativa:

$\mathrm{K}=-0.2673376812 \mathrm{e}-7$

$K\left(\lambda_{1}\right)=-0.1091299682 \mathrm{e}-7$

$K\left(\lambda_{2}\right)=-0.1578832193 \mathrm{e}-7$

$\mathrm{K}\left(\lambda_{3}\right)=-0.2661468867 \mathrm{e}-7$

Análise do conjunto de dados II

Considerando-se os dados de tempo de vida da tabela $3.15, a_{1}=116, b_{1}=1078, a_{2}=240$, $b_{2}=4900, a_{3}=291, b_{3}=5400, r=71, n-r=29, n=100, x=674.45, y=1118.88, R m=1343.65$. Leandro (1997) usou o mesmo procedimento para o algoritmo G-MH usado para o conjunto de dados I.

Os resultados obtidos foram:

\begin{tabular}{|l|l|l|l|}
\hline & $\lambda_{1}$ & $\lambda_{2}$ & $\lambda_{3}$ \\
\hline EMV & 0.10782 & 0.04896 & 0.0164 \\
\hline Confianca 95\% & $(0.0605 ; 0.15551)$ & $(0.0163,0.0816)$ & $(0.0072,0.1007)$ \\
\hline Cred 95\%[G-MH] & $(0.0875,0.1333)$ & $(0.0467,0.0511)$ & $(0.0514,0.0557)$ \\
\hline
\end{tabular}

Tabela 3.15. resultados da inferência para parâmetros do conjunto de dados II 
Os escalares de Ricci são dados por:

Caso Clássico:

$\mathrm{K}=-0.7766443283 \mathrm{e}-6$

$\mathrm{K}\left(\lambda_{1}\right)=-0.7499681526 \mathrm{e}-6$

$\mathrm{K}\left(\lambda_{2}\right)=-0.7816922967 \mathrm{e}-6$

$K\left(\lambda_{3}\right)=-0.6551525434 \mathrm{e}-6$

Caso Bayesiano com priori informativa:

$\mathrm{K}=-0.1997688765 \mathrm{e}-9$

$\mathrm{K}\left(\lambda_{1}\right)=-0.1821096604 \mathrm{e}-11$

$\mathrm{K}\left(\lambda_{2}\right)=-0.1975541401 \mathrm{e}-9$

$K\left(\lambda_{3}\right)=-0.1997242287 e-9$

Análise do conjunto de dados III

Neste exemplo desenvolveu-se a análise Bayesiana do modelo ACBVE em testes de vida acelerados com o modelo de potência com $P=2, V_{1}=1, V_{2}=2$ e $V_{3}=3$. Os dados usados são os da tabela 3.16 .

Para a análise Bayesiana consideraram-se as densidades a priori dadas por $\mathrm{N}_{1} \sim \mathrm{b}\left(15, \mathrm{c}_{1} / \mathrm{c}_{12}\right), \quad \mathrm{c}_{1} \sim \Gamma\left(\mathrm{a}_{1}, \mathrm{~b}_{1}\right), \quad \mathrm{c}_{2} \sim \Gamma\left(\mathrm{a}_{2}, \mathrm{~b}_{2}\right), \quad \mathrm{c}_{3} \sim \Gamma\left(\mathrm{a}_{3}, \mathrm{~b}_{3}\right) \quad$ e $\quad \mathrm{P} \sim \mathrm{N}\left(\mu_{0}, \sigma_{0}{ }^{2}\right), \quad$ onde $\mathrm{a}_{1}=5, \mathrm{~b}_{1}=72, \mathrm{a}_{2}=15, \mathrm{~b}_{2}=54, \mathrm{a}_{3}=3, \mathrm{~b}_{3}=41, \mu_{0}=2$ e $\sigma_{0}{ }^{2}=0.05$.

O procedimento adotado por Leandro (1997) foi o mesmo da análise de dados $\mathrm{I}$. Houve, nesse caso, o uso de duas parametrizações: $\left(c_{1}, c_{2}, c_{3}\right)$ e $\left(\theta_{1}=\ln c_{1}, \theta_{2}=\ln c_{2}, \theta_{3}=\operatorname{lnc} c_{3}\right)$. Os resultados foram

\begin{tabular}{|l|l|l|}
\hline & $\left(c_{1}, c_{2}, c_{3}\right)$ & $\left(\theta_{1}, \theta_{2}, \theta_{3}\right)$ \\
\hline$c_{1}$ & $(0.03116,0.09542)$ & $(0.04492,0.08001)$ \\
\hline$c_{2}$ & $(0.18867,0.36606)$ & $(0.20074,0.30751)$ \\
\hline$c_{3}$ & $(0.02288,0.10454)$ & $(0.04301,0.07839)$ \\
\hline$P$ & $(1.60068,2.46958)$ & $(1.56080,2.42738)$ \\
\hline
\end{tabular}

Tabela 3.16: resultados da inferência para parâmetros do conjunto de dados III

Os escalares de Ricci são dados por

Parametrização c

$\mathrm{K}=-0.1980894473 \mathrm{e}-5$

$\mathrm{K}\left(\mathrm{c}_{1}\right)=-0.1903494802 \mathrm{e}-5$

$K\left(c_{2}\right)=-0.9691099922 \mathrm{e}-6$

$K\left(c_{3}\right)=-0.1990965821 e-5$

Parametrização $\theta$

$\mathrm{K}=-0.2313476925 \mathrm{e}-4$

$K\left(\theta_{1}\right)=-0.2202543525 \mathrm{e}-4$

$\mathrm{K}\left(\theta_{2}\right)=-0.1704315793 \mathrm{e}-4$

$K\left(\theta_{3}\right)=-0.2259167125 e-4$

Conclusões 
Os resultados mostram que as curvaturas de Ricci diminuem quando usamos distribuições a posteriori informativas.

No caso III, curvaturas menores associam-se a parametrização que fornece menores intervalos de credibilidade.

Os reais motivos desses fatos exigiriam mais geometria diferencial no processo investigativo para esclarecer qual o significado geométrico do teorema de Bayes. Trata-se de um campo novo de pesquisa que poderia gerar novos algoritmos, modificando-se a estratégia do MCMC para incorporar a curvatura. 


\section{Capítulo 4 \\ Modelos Não Lineares}

\subsection{Curvaturas em dimensões maiores que dois}

O cálculo do escalar de Ricci em dimensões maiores que dois pode ser feito via tensor de Riemann, mas há outra alternativa: Há um tensor chamado tensor de Ricci que permite o cálculo de forma mais apropriada para nossos propósitos.

O tensor de Ricci é dado pela contração (ver apêndice 1) do tensor de Riemann

$\mathrm{R}_{\mathrm{ik}}=\mathrm{g}^{\operatorname{lm}} \mathrm{R}_{\mathrm{limk}}$

Dessa forma (ver Apêndice 1),

$\mathrm{R}_{\mathrm{ik}}=\partial \Gamma_{\mathrm{ik}}^{1} / \partial \mathrm{x}^{1}-\partial \Gamma_{\mathrm{il}}{ }^{1} / \partial \mathrm{x}^{\mathrm{k}}+\Gamma_{\mathrm{ik}}{ }^{1} \Gamma_{\mathrm{lm}}^{\mathrm{m}}{ }^{\mathrm{m}}-\Gamma_{\mathrm{il}}^{\mathrm{m}} \Gamma_{\mathrm{km}}{ }^{1}$

e

$\mathrm{K}=\mathrm{g}^{\mathrm{ik}} \mathrm{R}_{\mathrm{ik}}$

A vantagem de se calcular $R_{\mathrm{ik}}$ é que podemos distinguir o quanto cada parâmetro colabora na curvatura global do modelo estatístico. Por exemplo, no caso de 3 parâmetros temos:

$$
\begin{aligned}
& \mathrm{K}=\mathrm{g}^{11} \mathrm{~g}^{22} \mathrm{R}_{12}+\mathrm{g}^{11} \mathrm{~g}^{33} \mathrm{R}_{13}+\mathrm{g}^{22} \mathrm{~g}^{33} \mathrm{R}_{23}+\mathrm{g}^{11} \mathrm{~g}^{11} \mathrm{R}_{11}+\mathrm{g}^{22} \mathrm{~g}^{22} \mathrm{R}_{22}+\mathrm{g}^{33} \mathrm{~g}^{33} \mathrm{R}_{33} \\
& \mathrm{~K}_{1}=\mathrm{K}-\mathrm{g}^{22} \mathrm{~g}^{33} \mathrm{R}_{23} \\
& \mathrm{~K}_{2}=\mathrm{K}-\mathrm{g}^{11} \mathrm{~g}^{33} \mathrm{R}_{13} \\
& \mathrm{~K}_{3}=\mathrm{K}-\mathrm{g}^{11} \mathrm{~g}^{22} \mathrm{R}_{12}
\end{aligned}
$$

Notamos que $K_{1}$ apresenta uma soma de parcelas com o parâmetro $\theta_{1}$, ele representa todas as possiveis colaborações do parâmetro $\theta_{1}$ na curvatura escalar do modelo estatístico global. Vamos denominá-lo curvatura parcial $1 . \mathrm{K}_{2}$ e $\mathrm{K}_{3}$ têm significados análogos.

\subsection{Uso de curvaturas para discriminação de modelos de crescimento sigmóide}

Processos de crescimento que resultam em curvas de crescimento em forma de S são bastante comuns em biologia, agronomia, economia. Muitos modelos matemáticos apresentam tais características e são amplamente usados na análise de dados onde o crescimento é governado por considerações teóricas. Dentre tais modelos, citamos: modelo Logístico, Gompertz, Weibull, Morgan-Mercer- Flodin e Richards. Sejam as seguintes parametrizações para esses modelos:

$Y_{j}=\alpha\left[1+\exp \left(\beta-\gamma x_{j}\right)\right]+\varepsilon_{j}($ Logístico)

$Y_{j}=\alpha \exp \left[-\exp \left(\beta-\gamma x_{j}\right)\right]+\varepsilon_{j}($ Gompertz)

$Y_{j}=\alpha-\beta \exp \left[-\gamma\left(x_{j}\right)^{\delta}\right]+\varepsilon_{j}(M M F)$

$Y_{j}=\beta \gamma+\alpha\left(x_{j}\right)^{\delta}\left[\left(\gamma+\left(x_{j}\right)^{\delta}\right]^{-1}+\varepsilon_{j}\right.$ (Weibull)

$\mathrm{Y}_{\mathrm{j}}=\alpha\left[1+\exp \left(\beta-\gamma \mathrm{x}_{\mathrm{j}}\right)\right]^{-1 / \delta}+\varepsilon_{\mathrm{j}}$ (Richards) 
onde $\varepsilon_{\mathrm{j}}(\mathrm{j}=1 \ldots \mathrm{n})$, considerados variáveis aleatórias independentes e identicamente distribuida, normais com média zero e variância $\sigma^{2}, \varepsilon_{\mathrm{j}} \sim \mathrm{N}\left(0, \sigma^{2}\right)$.

\section{Exemplo}

Considere a análise dos dados obtidos por Heyes e Brown (1956), ver tabela 4.1, os quais se referem a quantidade de água presente em células de feijão (variável dependente y), obtida em diferentes pontos das raizes (variável independente $\mathrm{x}$ ).

\section{Estimação dos parâmetros}

Os parâmetros são obtidos por Método de Gauss-Newton com critério de convergência erro $=\left|\theta^{(n+1)}-\theta^{(n)}\right|<1$ e-10, onde $\theta$ são os parâmetros de interesse. Os resultados são dados por

A partir das estimativas dos parâmetros obtidos pelo método de Gauss-Newton apresentamos os gráficos de resíduos e escores normais para todos os modelos (figuras 4.24.6). As figuras 4.7-4.24 referem-se aos T-plots de Hills Smith e verossimilhanças profiles para todos os parâmetros de todos os modelos. T-plots mais retilíneos mostram maior comportamento linear do parâmetro e T-plots curvos mostram comportamento não linear. 
Tabela 4.1 - Dados de Heyes e Brown (1956).

\begin{tabular}{|c|c|}
\hline$x$ & $y$ \\
\hline 0.5 & 1.3 \\
1.5 & 1,3 \\
2.5 & 1.9 \\
3.5 & 3.4 \\
4.5 & 5.3 \\
5.5 & 7.1 \\
6.5 & 10.6 \\
7.5 & 16.0 \\
8.5 & 16.4 \\
9.5 & 18.3 \\
10.5 & 20.9 \\
11.5 & 20.5 \\
12.5 & 21.3 \\
13.5 & 21.2 \\
14.5 & 20.9 \\
\hline
\end{tabular}

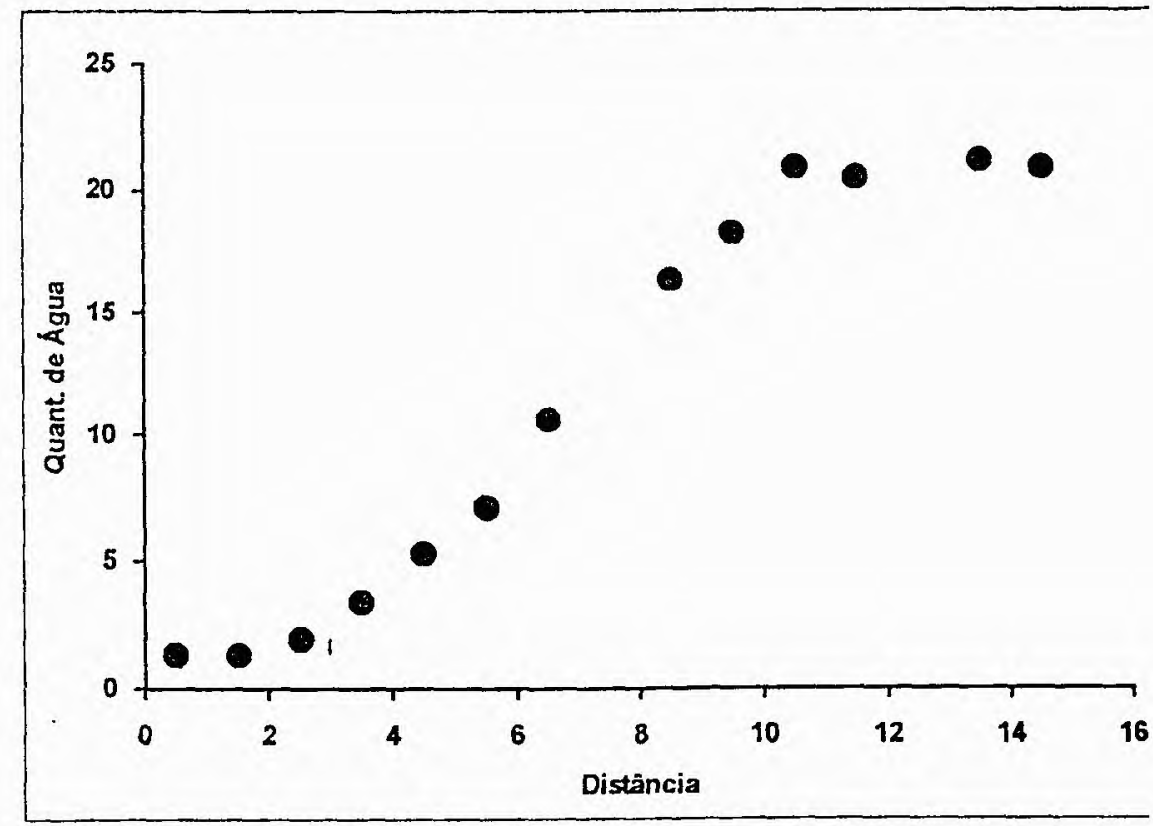

Fig 4.1. - Quantidade de Água versus Distância do Tronco. 

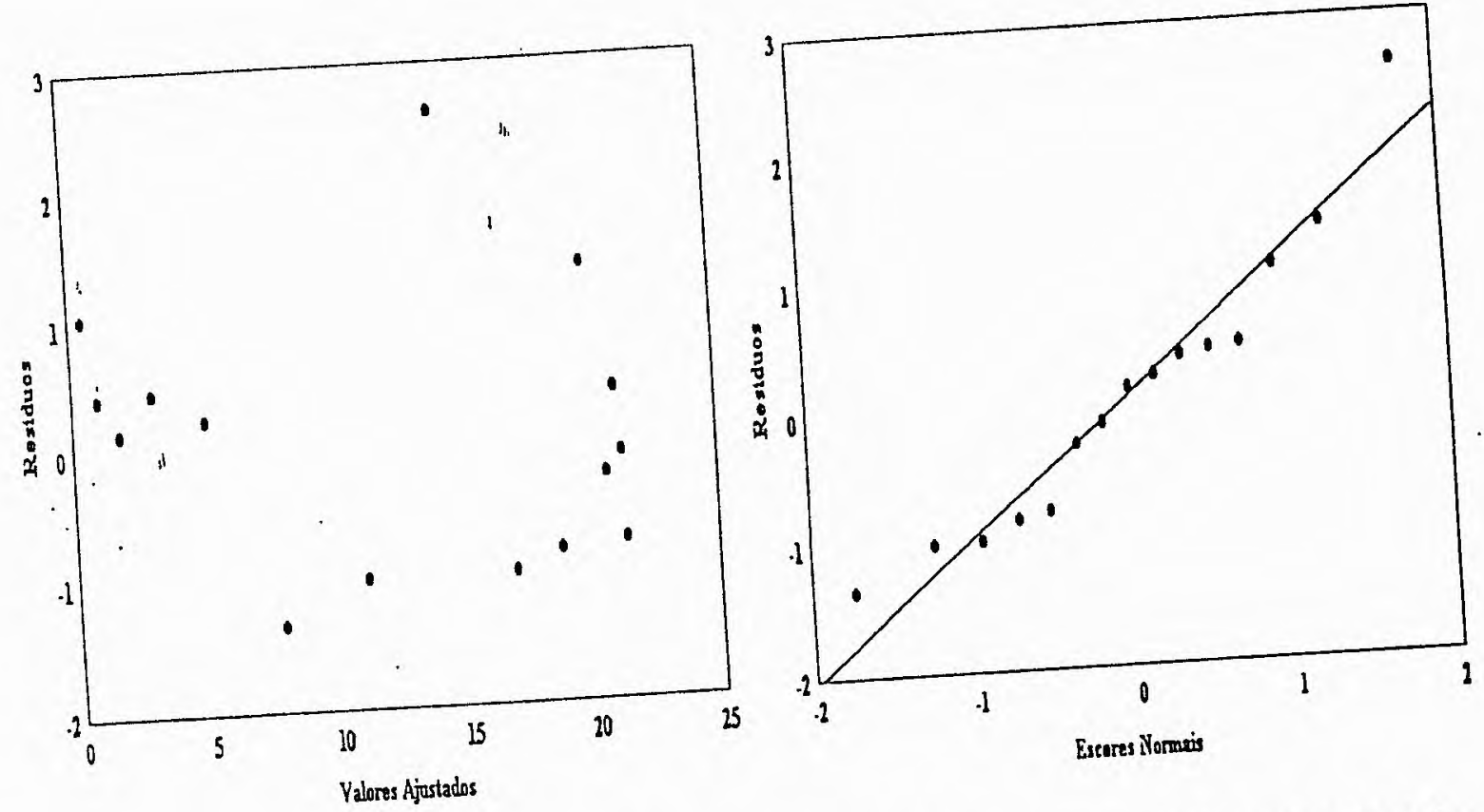

Fig 42. Gráficos de resíduos vs. valores ajustados e resíduos vs. escores normais-modeld 

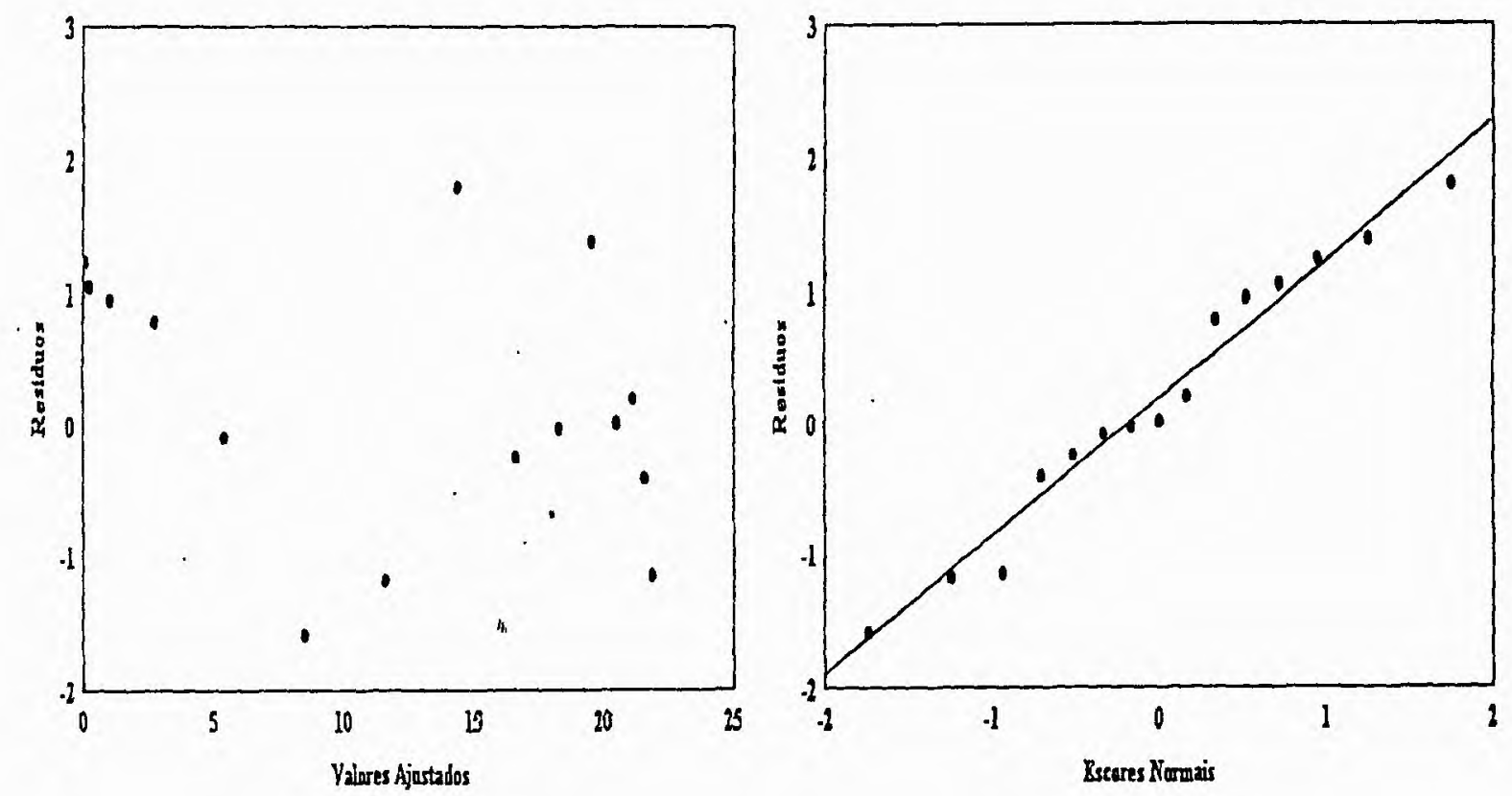

Fig 4.3. '- Gráficos de resíduos vs. valores ajustados e resíduos vs. escores normais-modelo Gompertz.
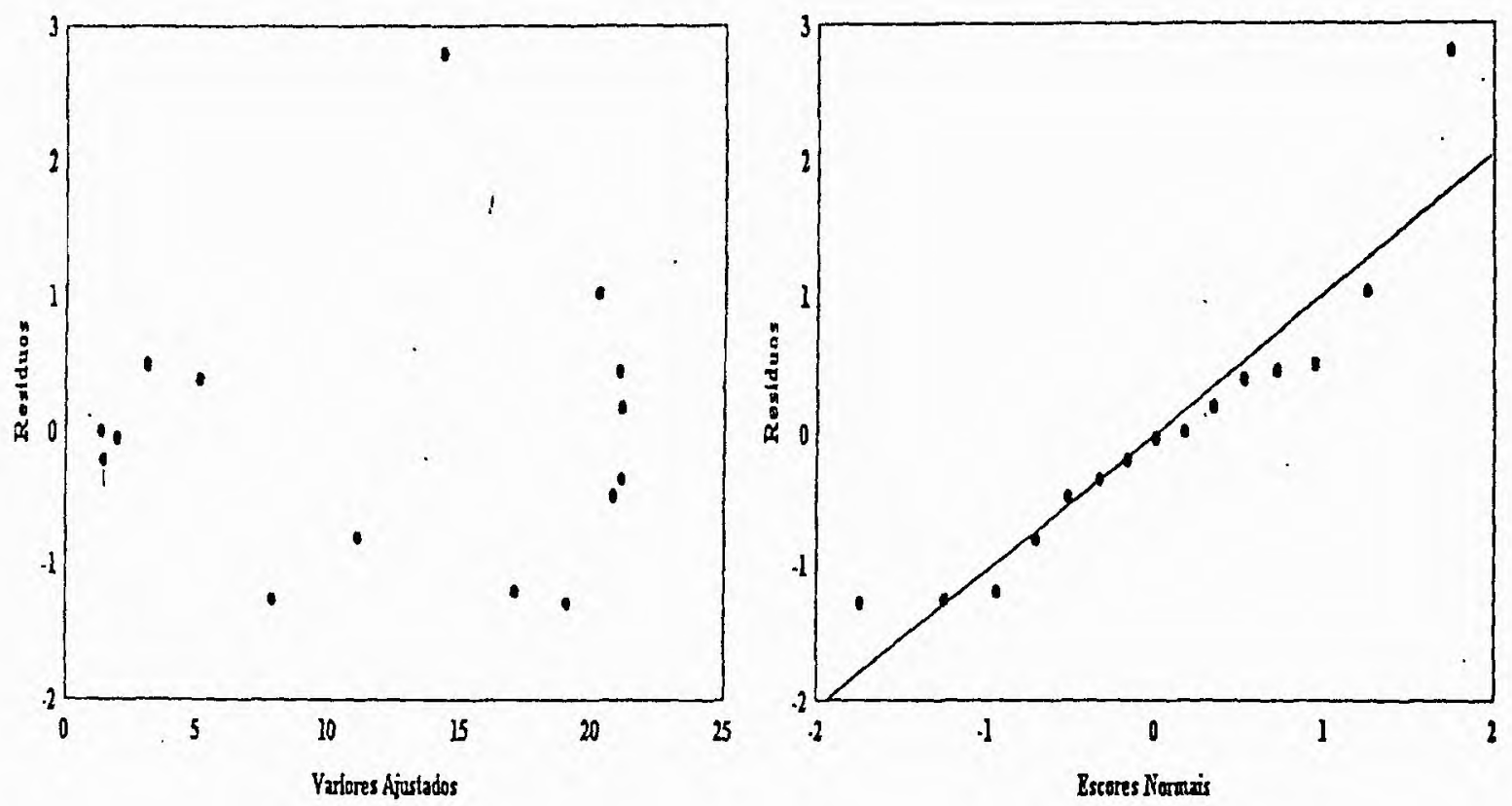

Fig 4.4. Gráficos de resíduos vs. valores ajustados e residuos vs. escores normais-modelo T. Weibull. 

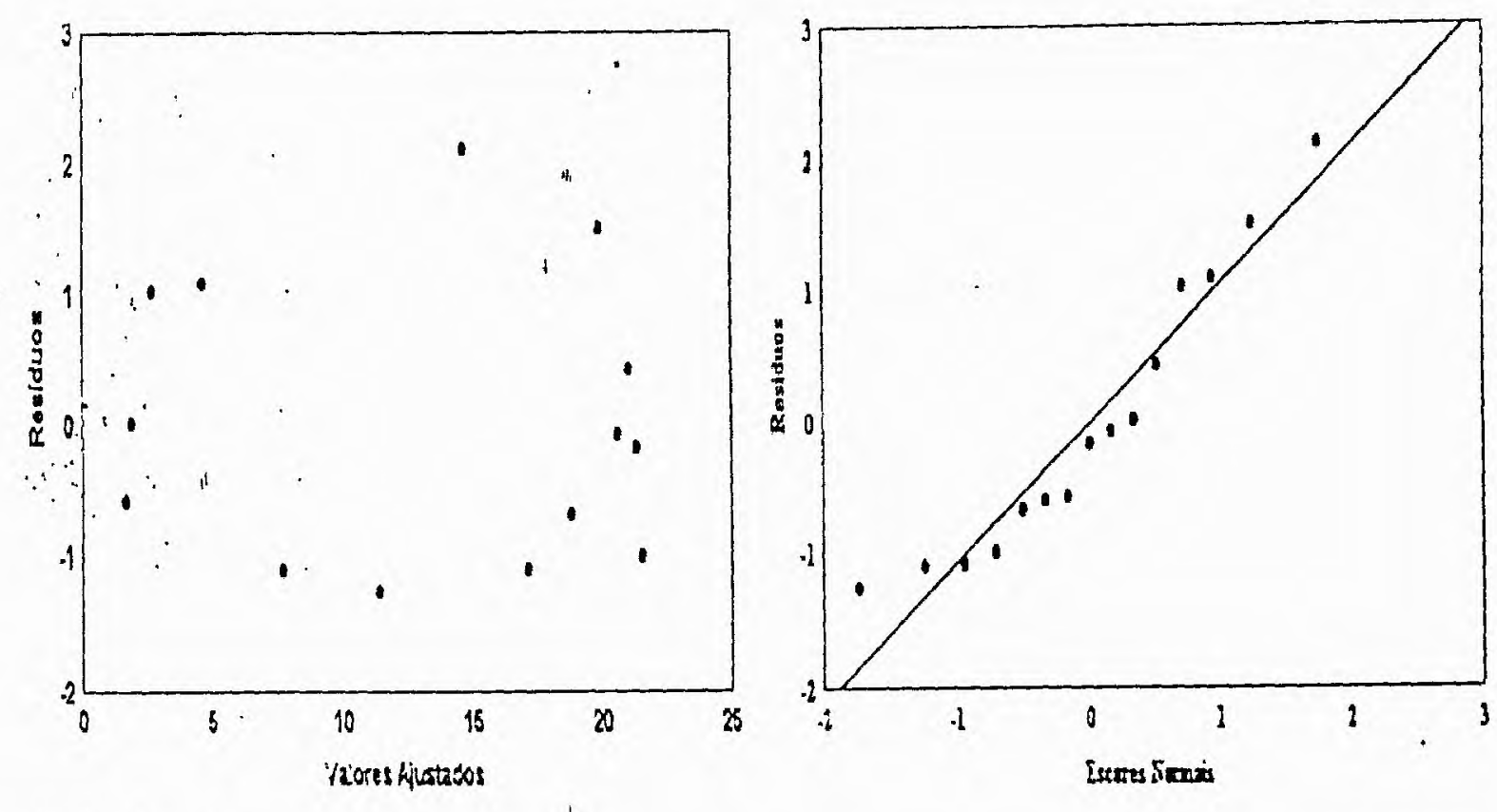

Fig 4.5. Gráficos de resíduos vs. valores ajustados e residuos vs. escores normais-modelo M.M.F.
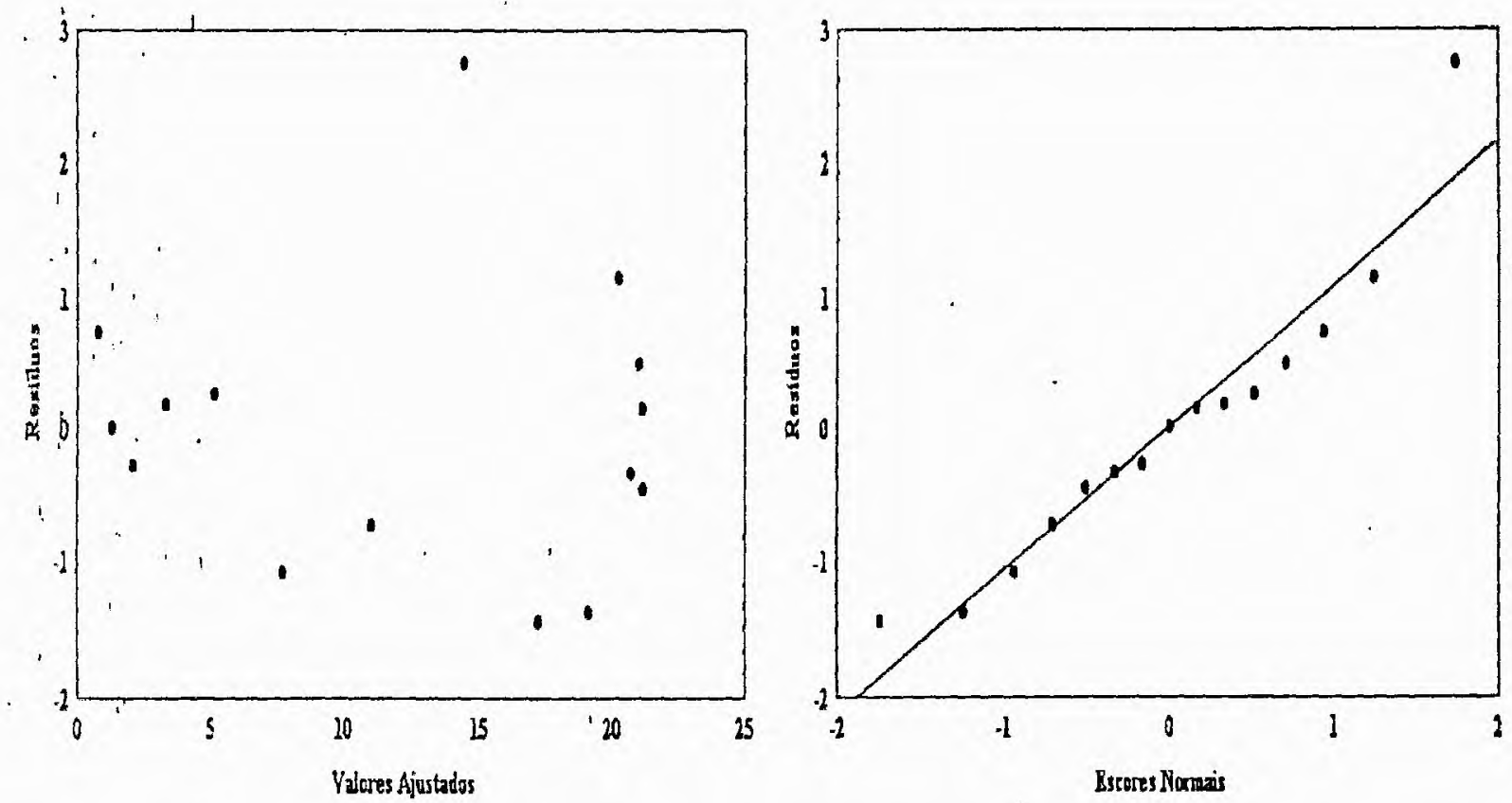

Fig 4.6. Gráficos de residuos vs. valores ajustados e resíduos vs. escores normais-modelo Richards. 

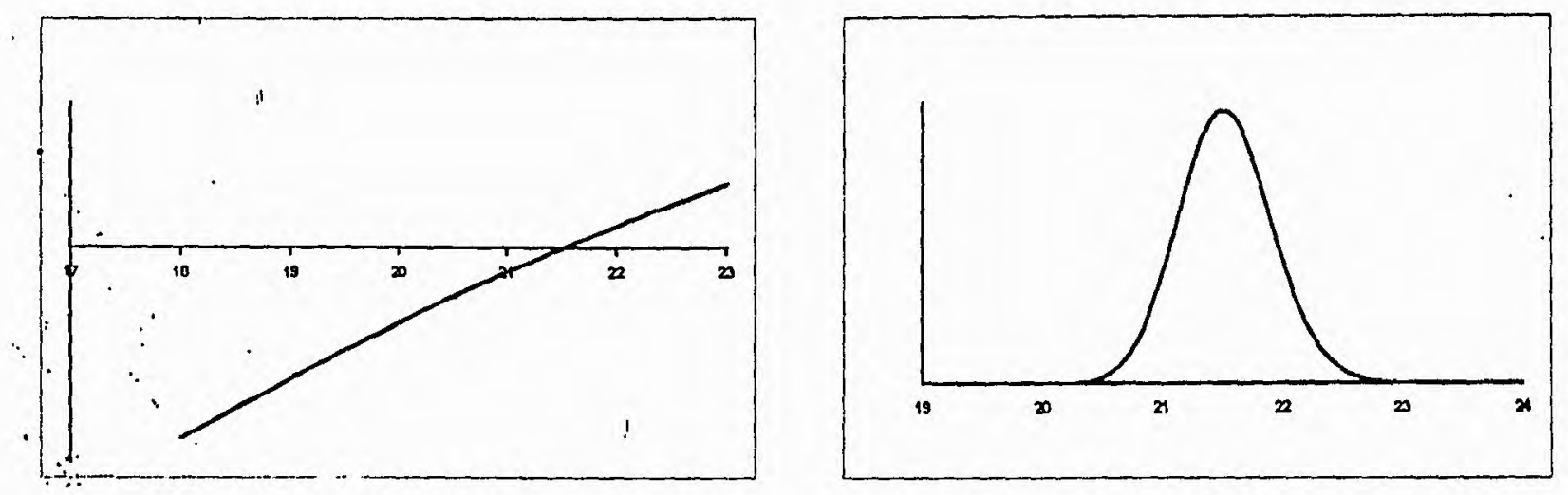

Fig 4.7. T-Plot de Hills Smith e Verossimilhança Profile - Modelo Logístico - Parâmetro $\alpha$.
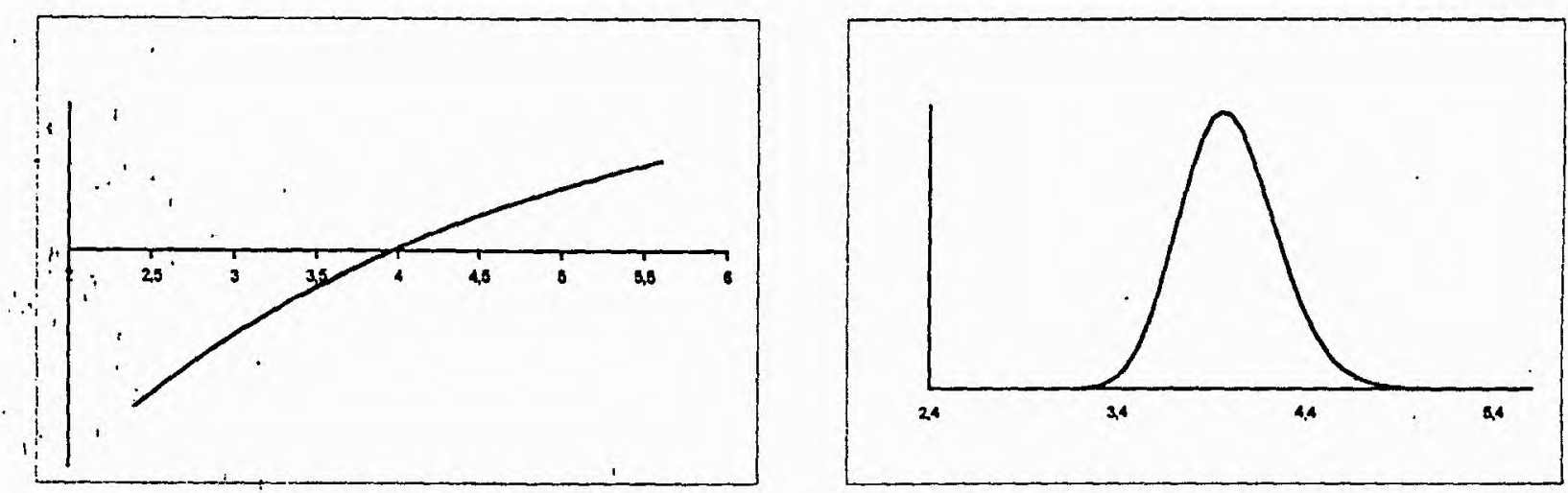

Fig 4.8. T-Plot de Hills Smith e Verossimilhança Profile - Modelo logístico - Parâmetro $\beta$.
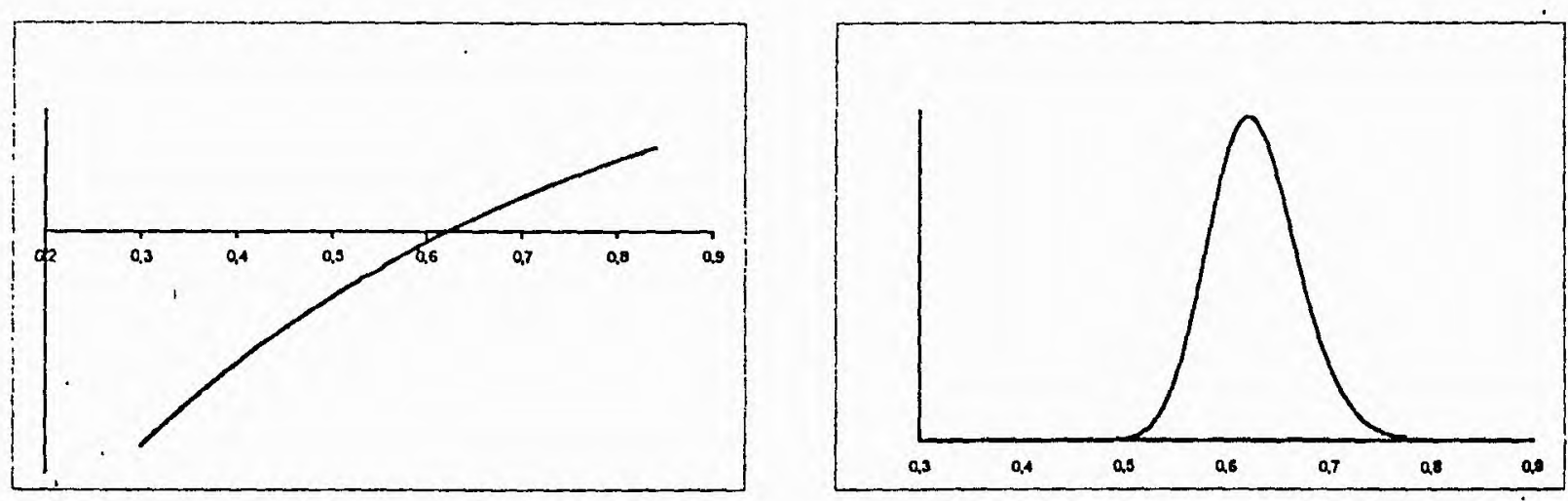

Fig 4.9. T-Plot de Hills Smith e Verossimilhança Profile - Modelo logístico - Parâmetro $\gamma$. 

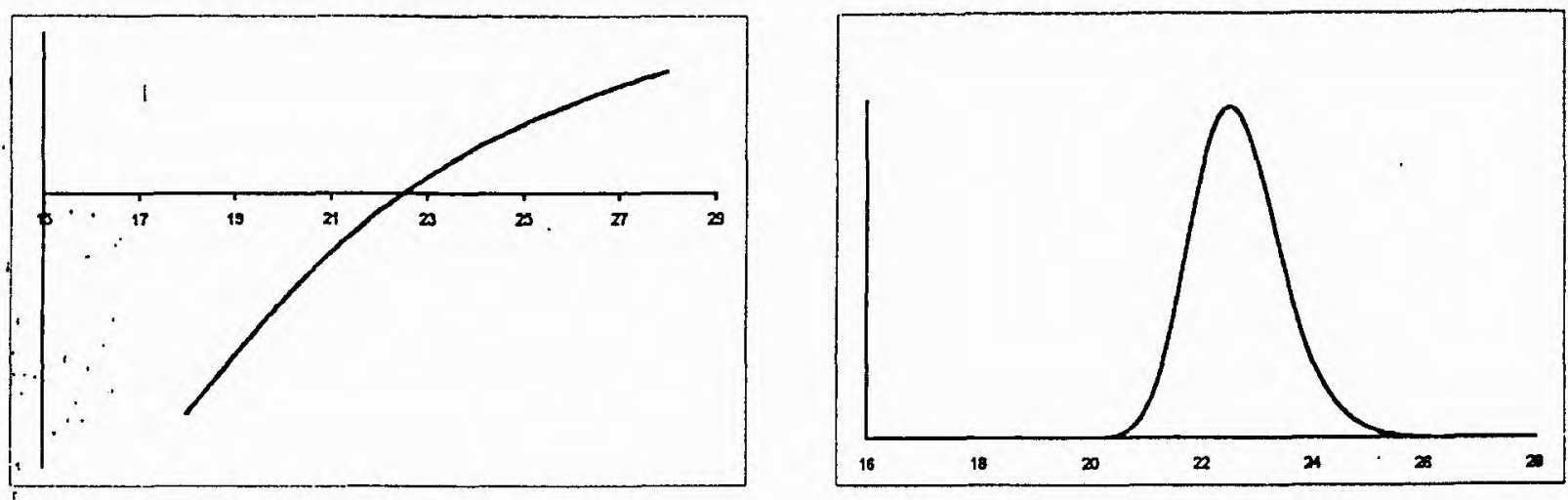

Fig 4.10. T-Plot de Hills Smith e Verossimilhança Profile - Modelo Gompertz - Parâmetro $\alpha$.
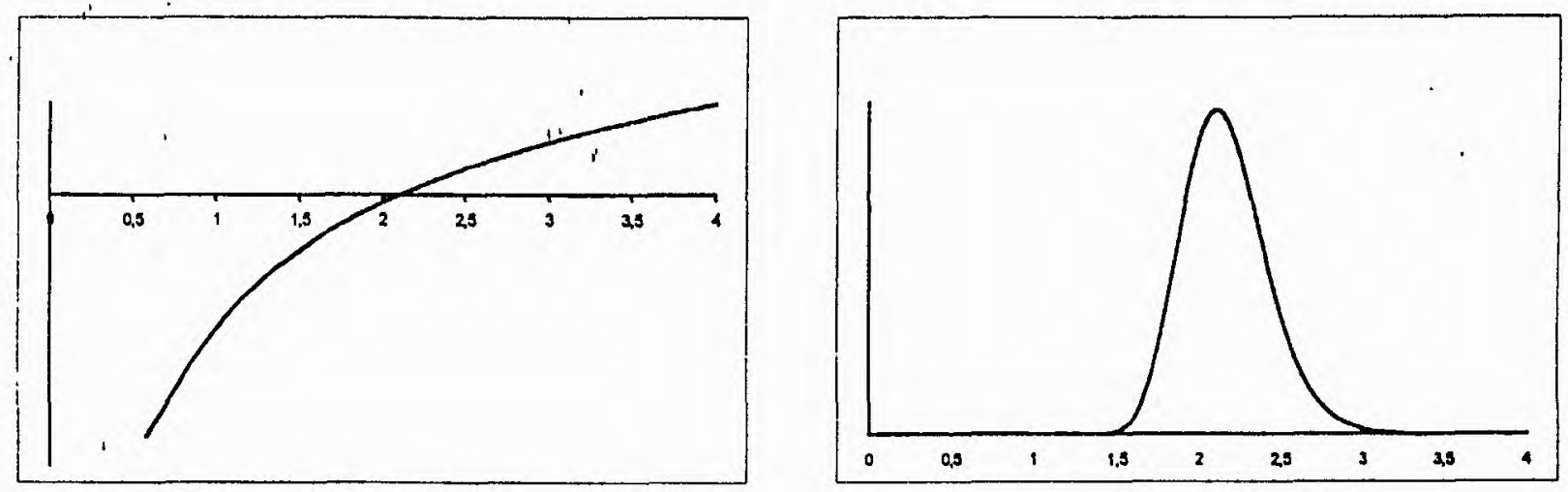

Fig 4.11. T-Plot de Hills Smith e Verossimilhança Profile - Modelo Gompertz - Parâmetro $\beta$.
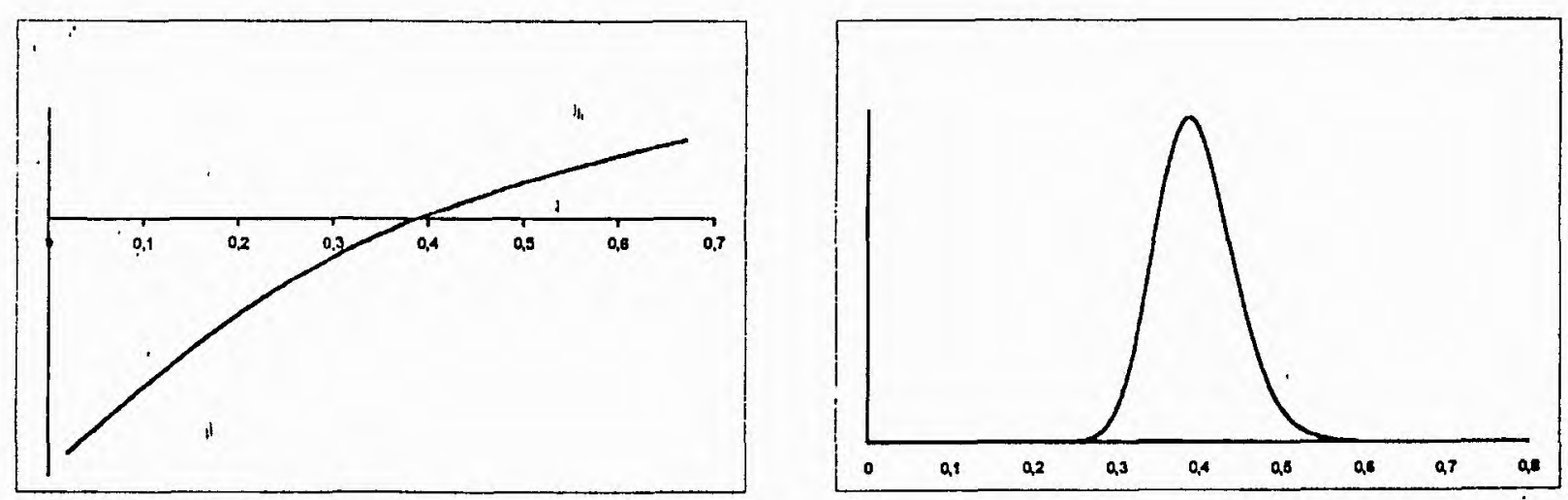

Fig 4.12. T-Plot de Hills Smith e Verossimilhança Profile - Modelo Gompertz - Parâmetro $\gamma$. 

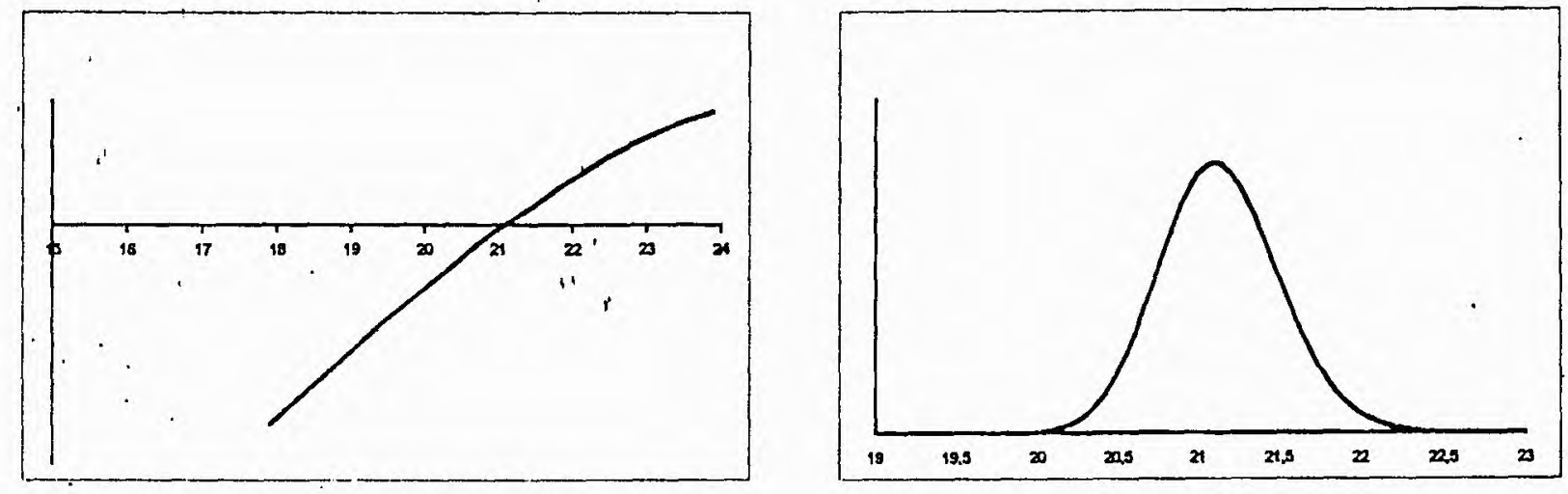

Fig 4.13. T-Plot de Hills Smith e Verossimilhança Profile - Modelo Weibull - Parâmetro $\alpha$.
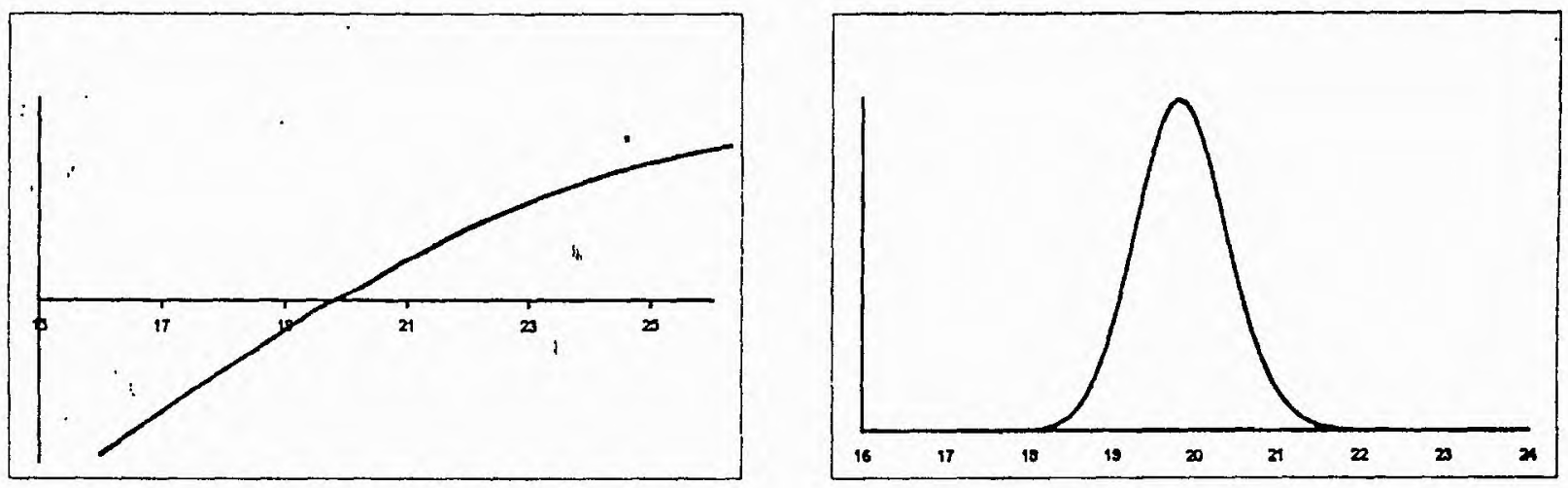

Fig 4.14. T-Plot de Hills Smith e Verossimilhança Profile - Modelo Wcibull - Parâmetro $\beta$.
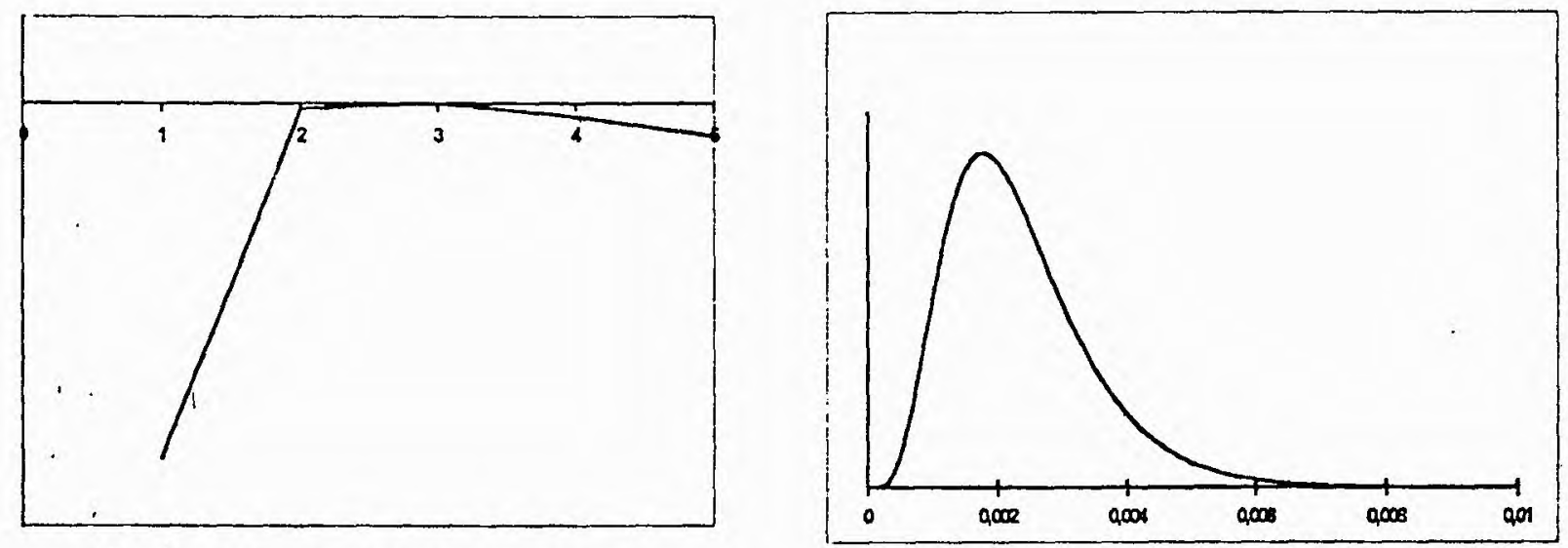

Fig 4.15. T-Plot de Hills Șmith e Verossimilhança Profile - Modelo Weibull - Parâmetro $\gamma$. 

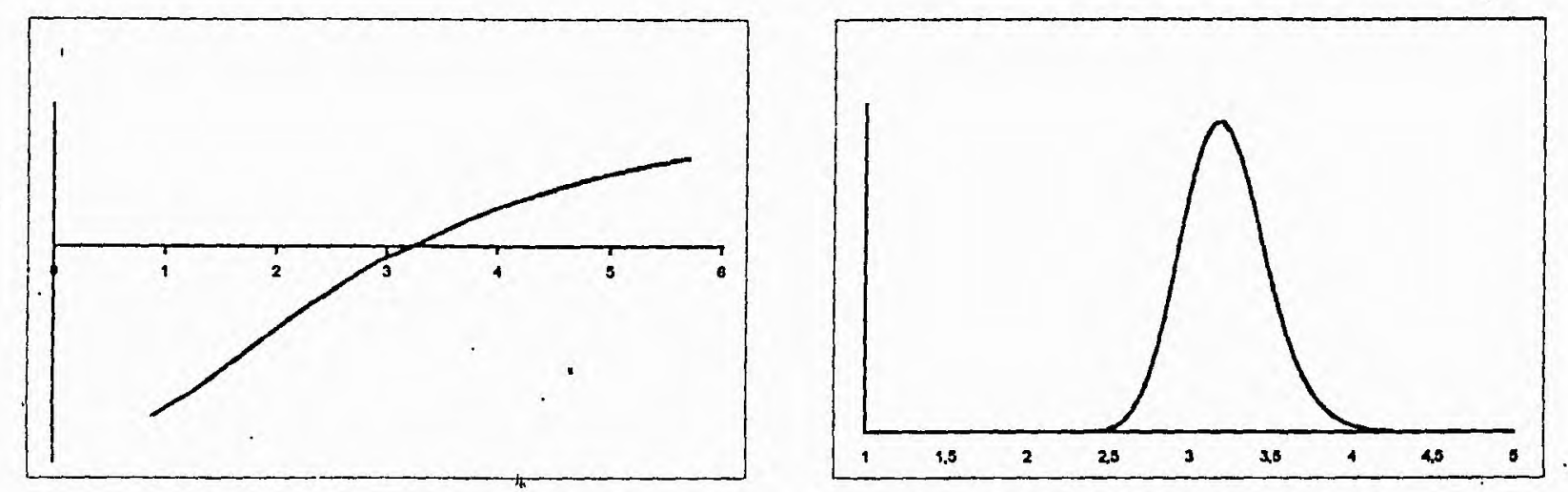

Fig 4.16. T-Plot de Hills Smith e Verossimilhança Profile - Modelo Tipo Weibull - Parâmetro $\delta$.
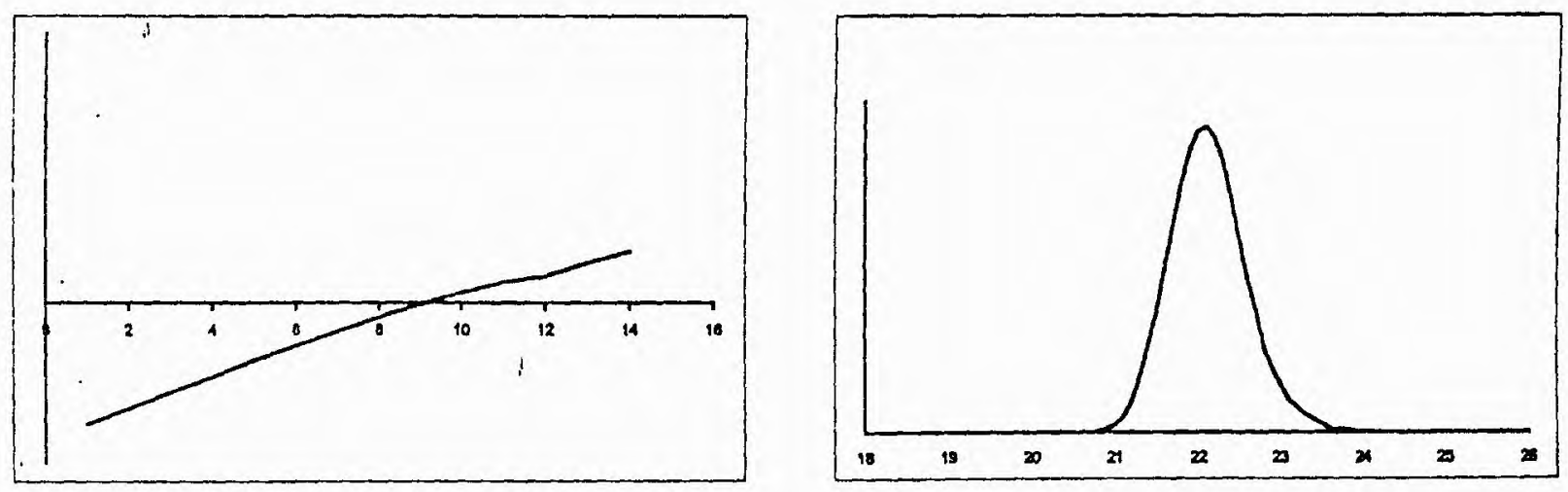

Fig 4.17. T-Plot de Hills Smith e Verossimilhança Profile - Modelo M.M.F. - Parâmetro $\alpha$.
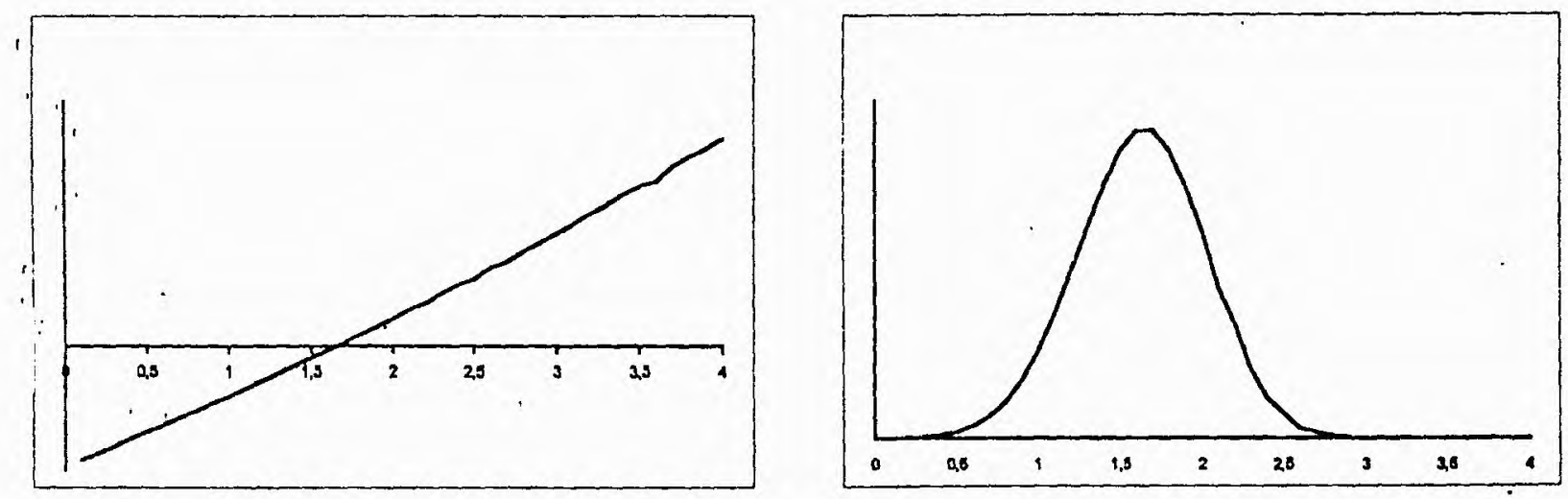

Fig 4.18. T-Plot de Hills Smith e Verossimilhança Profile - Modelo M.M.F. - Parâmetro $\beta$. 

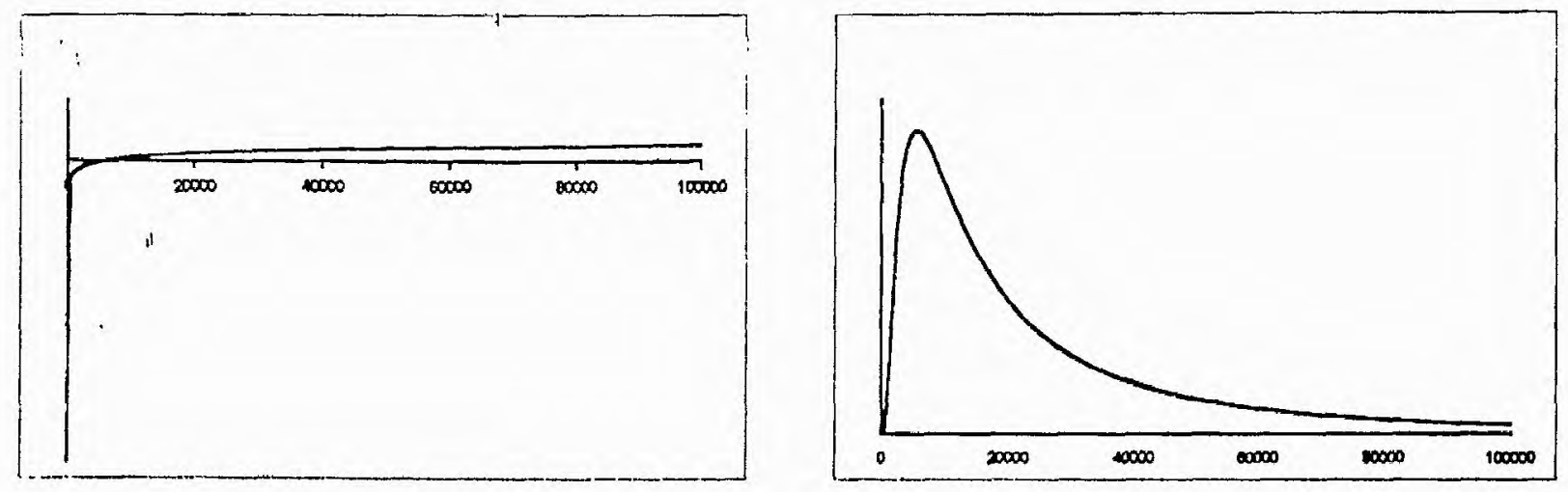

Fig 4.19. - T-Plot de Hills Smith e Verossimilhança Profile - Modelo M.M.F. - Parâmetro $\gamma$.
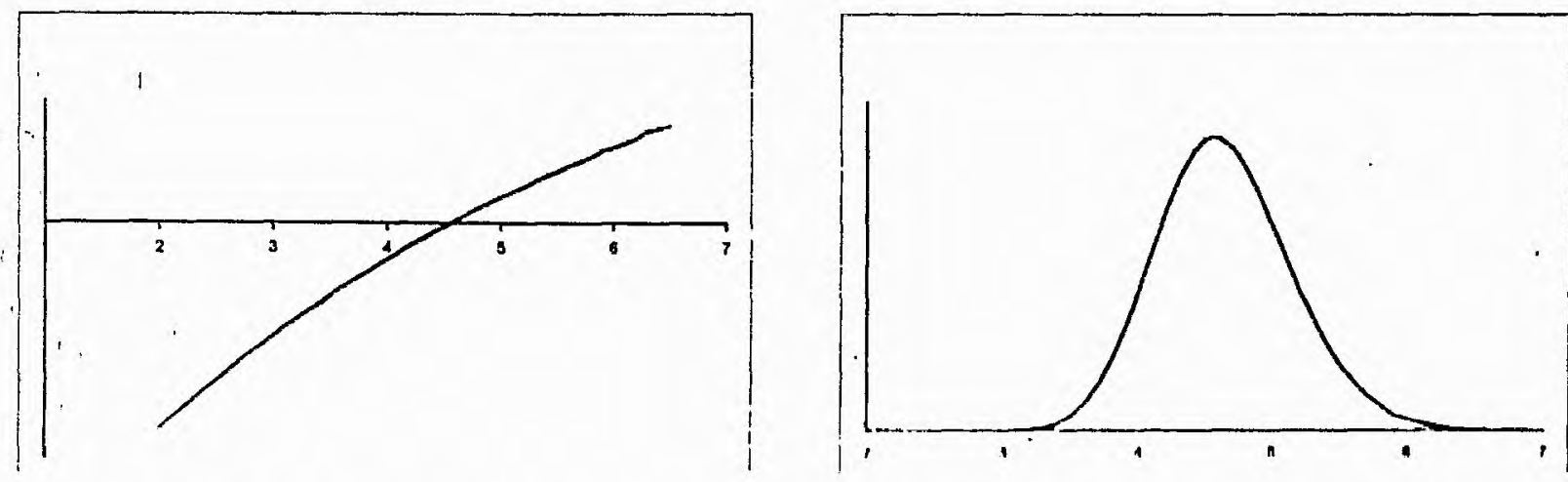

Fig 4.20. 'T-1'lot de llills Sunith e Verossimilhança l'rofile - Mlodelo M.M.k. - P'arânetro $\delta$.
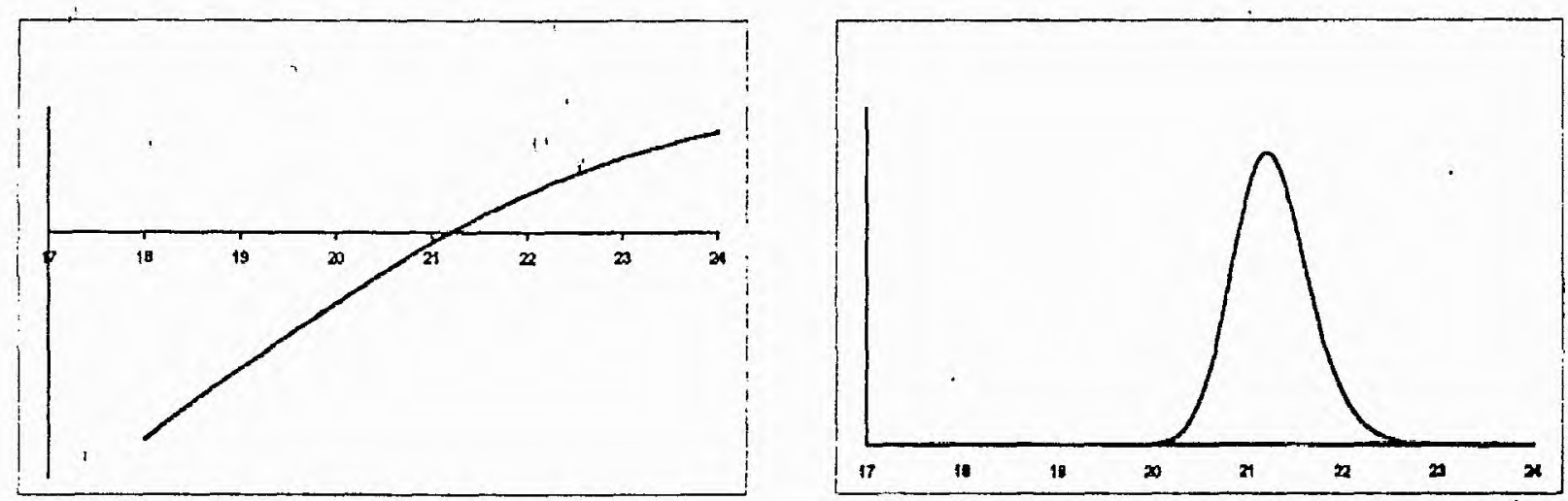

Fig 4.21. T-Plot de Hills Smith e Verossimilhança Profile - Modelo Richards - Parâmetro $\alpha$. 

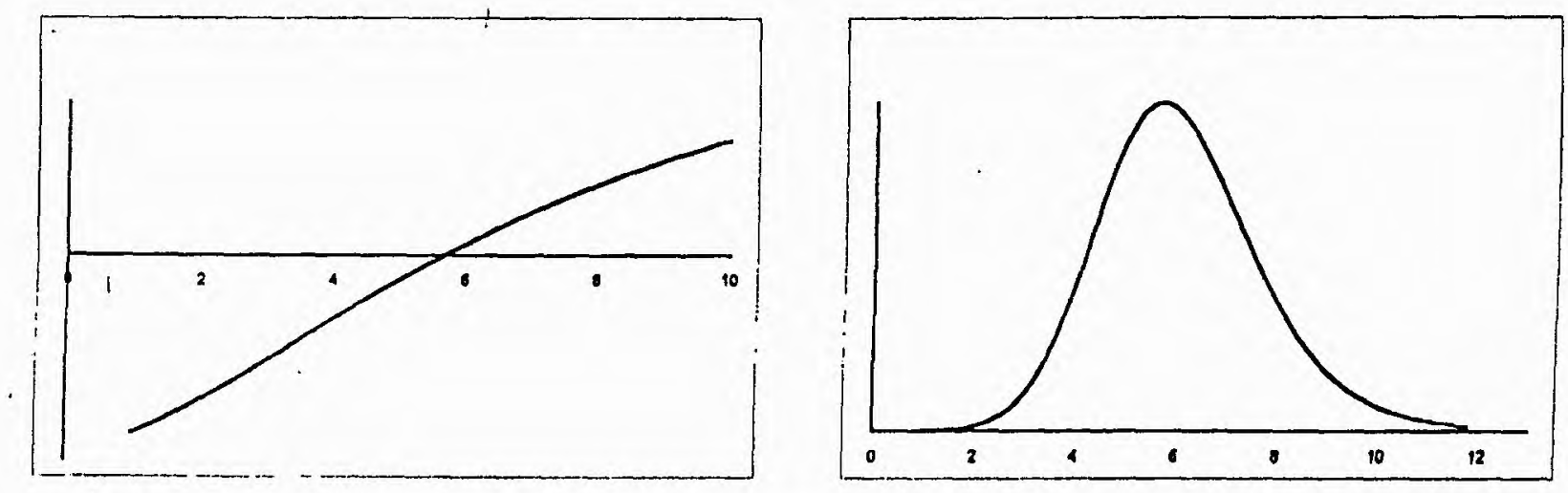

Fig 4.22. T-Plot de Hills Sinith e Verossimilhanca Profile - Modelo Richards - Parâmetro $\beta$
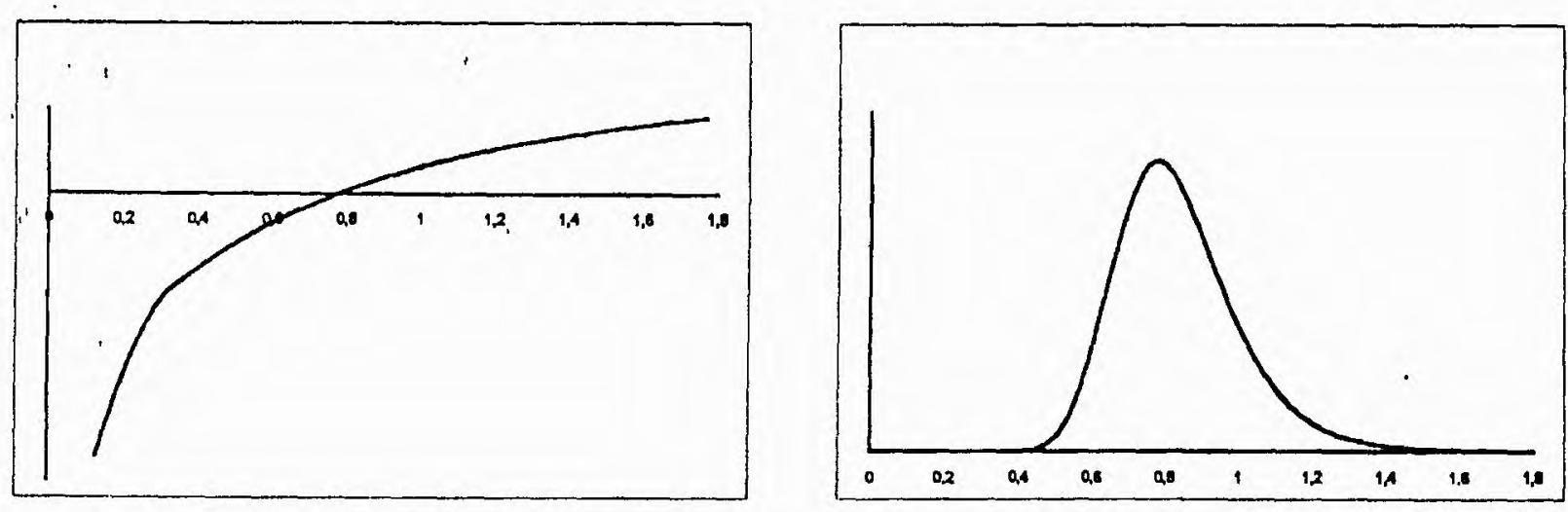

Fig 4.23.. T-Plot de Hills Smith e Verossimilhanca Profile - Modelo Richards - Parâmetro $\gamma$
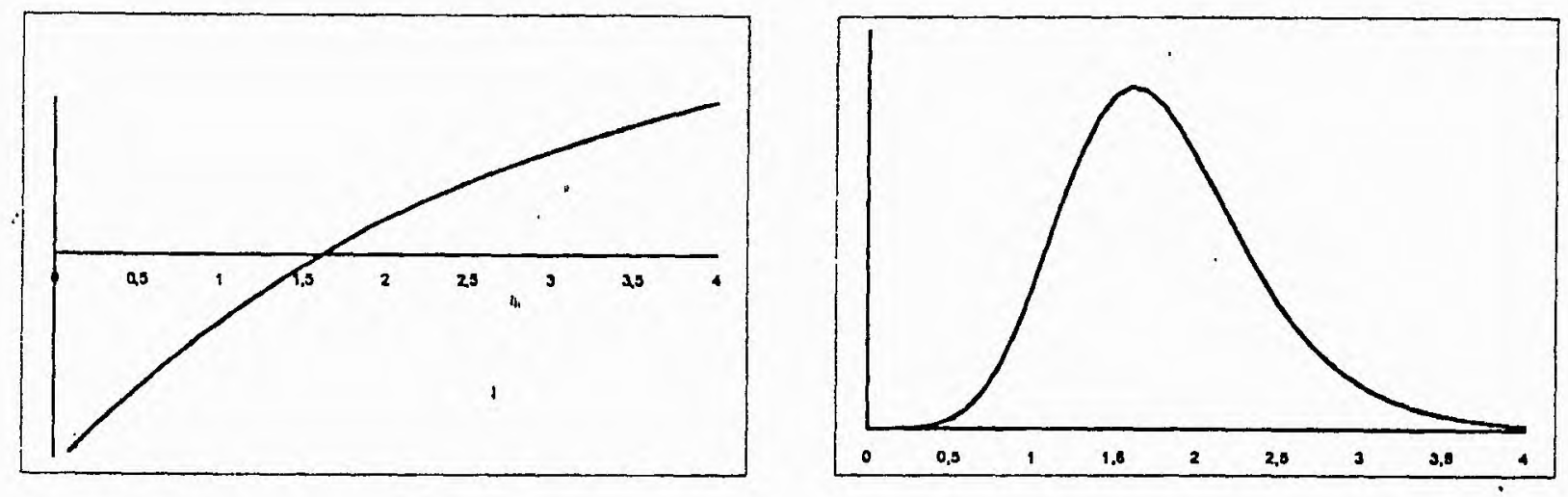

Fig 4.24. T-Plot de Hills Smith e Verossimilhanca Profile - Modelo Richards - Parâmetro $\delta$ 


\subsection{Avaliação da Não Linearidade}

Buscamos qual o melhor modelo possivel entre os cinco propostos. Para isso devemos quantificar o comportamento não linear. Uma forma de fazê-lo é calculando-se as medidas de curvatura de Bates e Watts para cada modelo:

\begin{tabular}{|l|l|l|}
\hline Modelo & Curvatura intrínseca & Curvatura Paramétrica \\
\hline Logístico & 0,1070 & 0,3717 \\
\hline Gompertz & 0,2318 & 0,8799 \\
\hline Weibull & 0,2319 & 13,2534 \\
\hline MMF & 0,2102 & 24,9338 \\
\hline Richards & 0,2948 & 4,2680 \\
\hline
\end{tabular}

Tabela 4.2. Curvaturas intrínseca e paramétrica para os modelos de crescimento sigmóide

Tais medidas apresentam alta não linearidade paramétrica. Tal fato indica que pelo menos um parâmetro no modelo comporta-se de forma não linear.

Duas alternativas bastante usadas para esse diagnóstico seriam a medida de vício e de assimetria. Tais estratégias requerem considerável esforço computacional. Mazucheli (1995) calculou as medidas de Kass Slate (ver Apêndice) para os modelos e os resultados foram:

\begin{tabular}{|l|l|}
\hline Modelo & Medidas Globais de Não-Normalidade \\
\hline Logistico & 0.2027 \\
\hline Gompertz & 0.2410 \\
\hline Weibull & 32.2674 \\
\hline MMF & 122.8839 \\
\hline Richards & 5.3712 \\
\hline
\end{tabular}

Tabela 4.3.Medidas de Kass Slate para os dados de crescimento Sigmóide

O cálculo dos escalares de Ricci fornece os resultados

\begin{tabular}{|l|l|}
\hline Modelo & K \\
\hline Logistico & $-.2101808695 \mathrm{e}-3$ \\
\hline Gompertz & $-.2180767052 \mathrm{e}-3$ \\
\hline Weibull & $-.6824808605 \mathrm{e}-2$ \\
\hline MMF & $.1773229389 \mathrm{e} 18$ \\
\hline Richards & $-.9195604489 \mathrm{e}-3$ \\
\hline
\end{tabular}

Tabela 4.4: Escalares de Ricci para os dados de crescimento sigmóide

Observamos que há grande compatibilidade entre os escalar de Ricci e as medidas globais de Não Linearidade, no sentido de ambas darem o mesmo diagnóstico, permitindo uma classificação dos modelos quanto a sua não linearidade. Por ordem crescente de não linearidade temos: o modelo logístico, o modelo Gompertz, Richards, Weibull e MMF.

A seguir seria interessante detectar quais o parâmetros responsáveis pelo comportamento não linear. Isso pode ser detectado pelos T-Plot de Hills Smith e Verossimilhança profile, cujos gráficos se encontram nas páginas anteriores.

Chamamos de curvatura parcial do parâmetro $\theta_{1}$ a soma de todos os termos de curvatura que envolvem o parâmetro em questão $\theta_{1}$. (ver secção 4.1)

O cálculo das curvaturas parciais fornecem os seguintes resultados nesse caso: 


\begin{tabular}{|l|l|l|l|l|l|}
\hline & Logístico & Gompertz & Weibull & MMF & Richards \\
\hline$\alpha$ & $-.3773969 \mathrm{e}-4$ & $-.1205799 \mathrm{e}-4$ & $-.6885042 \mathrm{e}-2$ & $.1773229 \mathrm{e} 18$ & $-.2841368 \mathrm{e}-3$ \\
\hline$\beta$ & $-.9180733 \mathrm{e}-4$ & $-.1492831 \mathrm{e}-3$ & $-.6962106 \mathrm{e}-2$ & $.1773229 \mathrm{e} 18$ & $-.2441871 \mathrm{e}-3$ \\
\hline$\gamma$ & $-.2098542 \mathrm{e}-3$ & $-.2175683 \mathrm{e}-3$ & $.6824808 \mathrm{e}-2$ & $-3963430 \mathrm{e} 10$ & $.9198202 \mathrm{e}-3$ \\
\hline$\delta$ & & & $-.1504245 \mathrm{e}-3$ & $-.177322 \mathrm{e} 18$ & $.9193122 \mathrm{e}-3$ \\
\hline
\end{tabular}

Tabela 4.5.Curvaturas parciais para os modelos de crescimento sigmóide

Comparando-se as curvaturas de Ricci dos parâmetros com os gráficos t Plots de Hills e verossimilhança profile temos uma boa coerência. Parâmetros que curvam mais o modelo estatístico são de certa forma os parâmetros responsáveis pela não Linearidade dos mesmos modelos.

O diagnóstico da não Linearidade via tensor de Ricci parece bastante adequado para detectar quais parâmetros são mais responsáveis pelo comportamento não linear desse modelo. Isso é feito pelo cálculo das curvaturas parciais. 


\subsection{Casos de Reparametrizações em modelos não lineares}

Dados para a taxa de reação da isomerização catalítica do $\mathrm{n}$ pentano para 0 isopentano são dados em Carr (1960). Trata-se de um processo quimico onde um complexo químico é transformado em unidades mais simples chamadas isômeros. A taxa de reação depende de vários fatores, tais como as pressões parciais dos produtos e a concentração de catalisador.

O modelo não linear para isomerização é o de Hougen-Watson:

$$
f(x, \theta)=\theta_{1} \theta_{3}\left(x_{2}-x_{3}\right) /\left[(1,632)\left(1+\theta_{2} x_{1}+\theta_{3} x_{2}+\theta_{4} x_{3}\right)\right]
$$

onde $\mathrm{f}$ é a taxa de reação e $\mathrm{x}_{1}, \mathrm{x}_{2}, \mathrm{x}_{3}$ são as pressões parciais de hidrogênio, isopentano e n-pentano respectivamente.

Profiles tplots (figura 4.25) para cssa parametrização mostram-se bastante ruins. Isso mostra que o modelo está fornecendo pouca informação a respeito dos dados. Químicos têm tentado melhorar o quadro através da obtenção de mais resultados experimentais.

Uma outra alternativa é usar uma reparametrização:

$\beta_{1}=1 / \theta_{1} \theta_{2}$

$\beta_{2}=\theta_{2} / \theta_{1} \theta_{2}$

$\beta_{3}=\theta_{3} / \theta_{1} \theta_{2}$

$\beta_{4}=\theta_{4} / \theta_{1} \theta_{2}$

Dessa forma, temos $f(x, \beta)$ :

$$
f(x, \beta)=\left(x_{2}-x_{3}\right) /\left[(1.632)\left(\beta_{1}+\beta_{2} x_{1}+\beta_{3} x_{2}+\beta_{4} x_{3}\right)\right]
$$

esta forma de reparametrização é recomendada por Ratkowsky (1985) para modelos que possuem razão de polinômios. A estimativa de máxima verossimilhança é dada por $\beta^{+}=(0,739 ; 0,0523 ; 0,0278,0,123)$. Os profiles $t$ plots (figura 4.26) são muito melhores e mostram que as regiões obtidas por aproximação linear são muito próximas das exatas.

Para esse modelo os arrays de curvatura de Bates e Watts são dados por:

$$
\begin{aligned}
& C_{\theta 1}=[0,-0.110,-0.256,0.361,-0.064,1.762,-18.157,8.180,-48.275,119.610] \\
& C_{\theta 2}=[0,-0.032,0,0,-0.264,0.269,-4.898,0.008,-0.004,0.008] \\
& C_{\theta 3}=[0,0,-0.032,0,0.013,-0.102,-0.004,0.413,-4.919,0.009] \\
& C_{\theta 4}=[0,0,0,-0.032,0.020,-0.004,-0.102,-0.001,0.265,-9.826]
\end{aligned}
$$

Para a reparametrização $\beta$ os arrays de curvatura de Bates e Watts são dados por

$$
\begin{aligned}
& C_{\beta 1}=[-0.069,0.011,-0.007,0.006,-0.062,0.001,0.004,-0.060,0.003,-0.052] \\
& C_{\beta 2}=[0.0011,-0.062,0.001,0.004,-0.032,-0.0016,0.007,-0.015,0.005,-0.039] \\
& C_{\beta 3}=[-0.007,0.001,-0.060,0.003,-0.016,-0.015,0.005,0.018,-0.005,-0.035] \\
& C_{\beta 4}=[0.006,0.004,0.003,-0.052,0.007,0.005,-0.039,-0.005,-0.035,-0.088]
\end{aligned}
$$



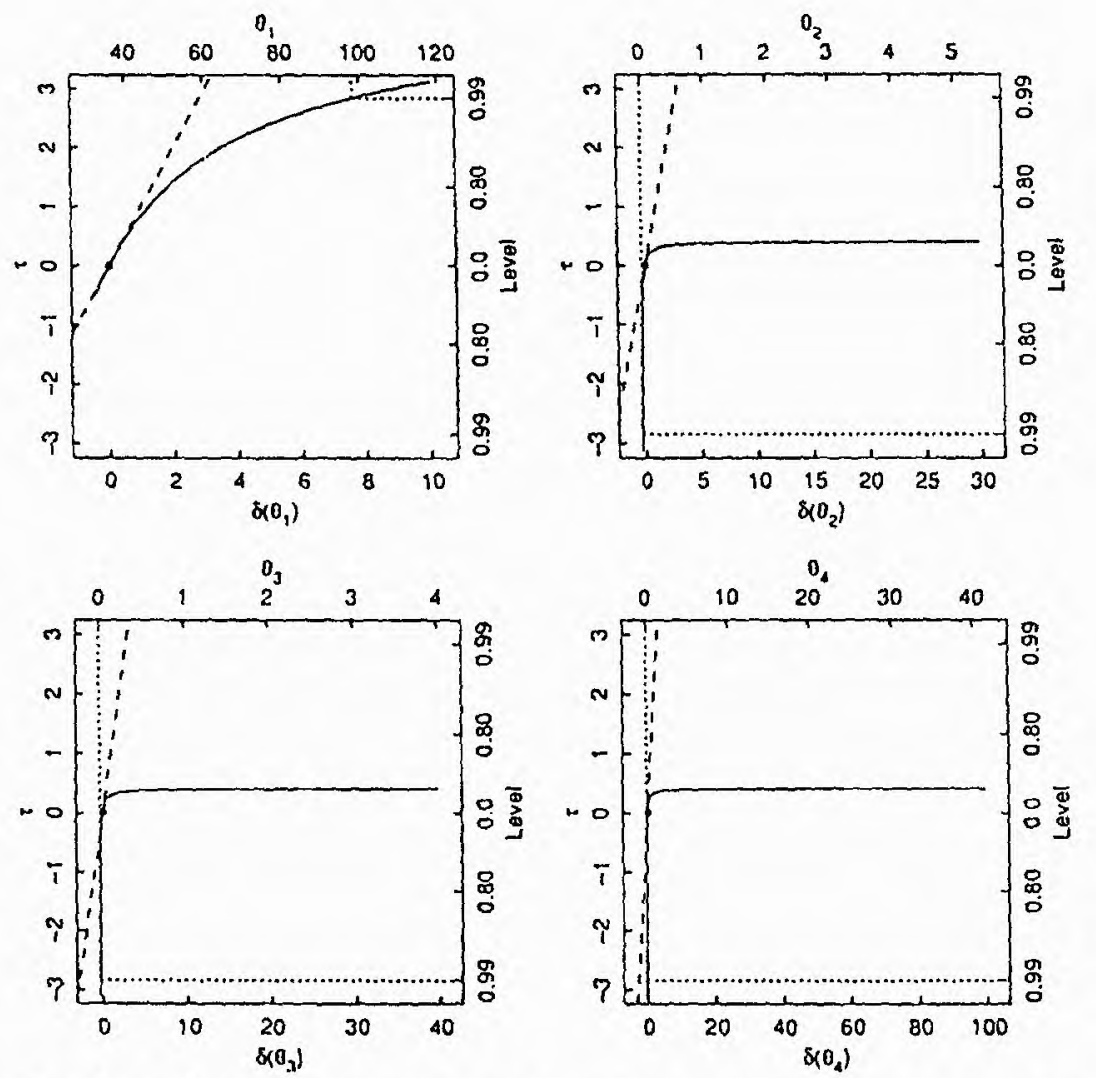

Figura 4.25. Profile t plots (linha contínua) para isomerização na parametrização $\theta$
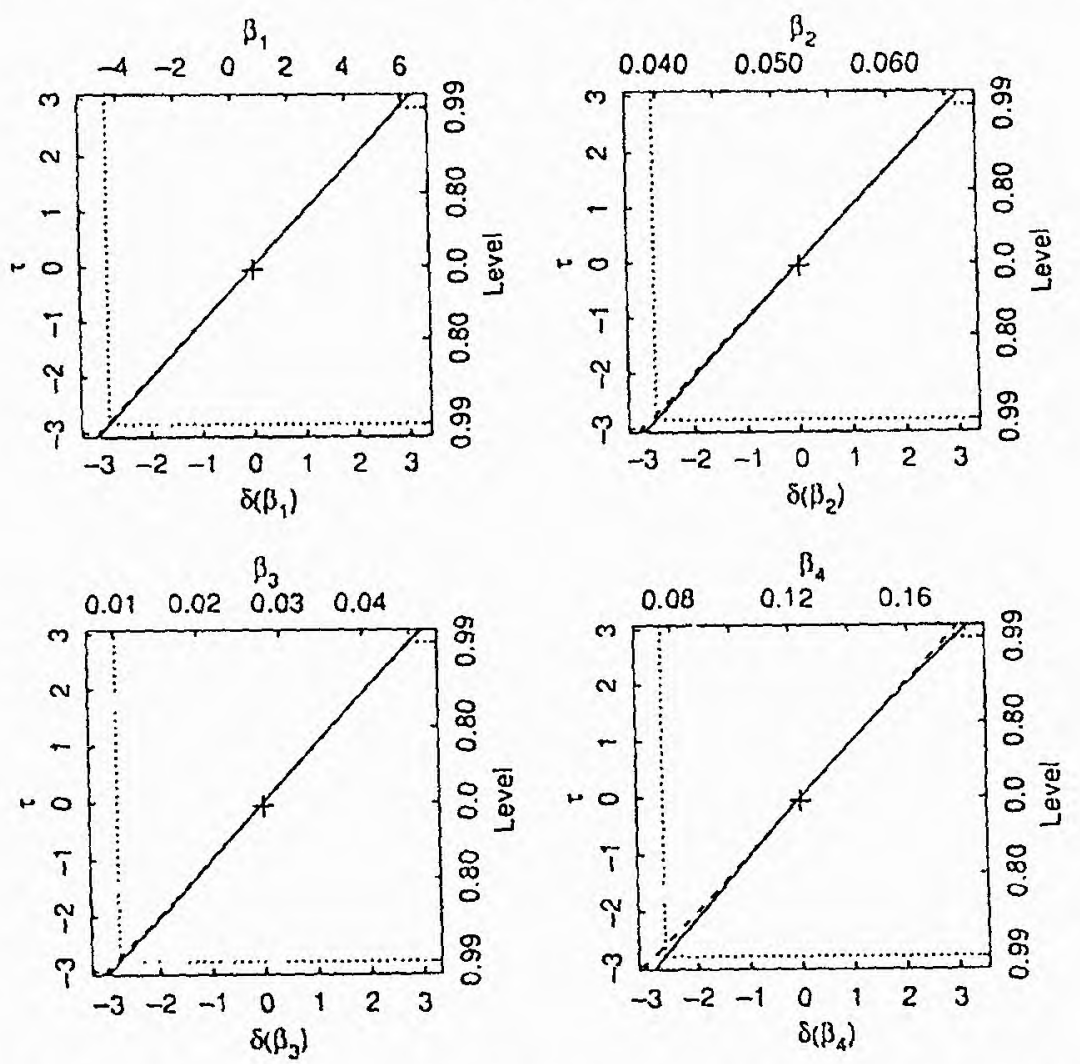

Figura 4.26. Profile t plots (linha contínua) para isomerização na parametrização $\beta$ 
Notamos que houve redução de curvatura e da não-linearidade, em alguns casos de forma drástica.

O cálculo dos escalares de Ricci fornecem os mesmos resultados qualitativos:

\begin{tabular}{|l|l|}
\hline & \\
\hline$K$ & $.3322056817 \mathrm{e}-2$ \\
\hline$K\left(\theta_{1}\right)$ & $.4837599983 \mathrm{e}-5$ \\
\hline$K\left(\theta_{2}\right)$ & $.3321938892 \mathrm{e}-2$ \\
\hline$K\left(\theta_{3}\right)$ & $.3321990407 \mathrm{e}-2$ \\
\hline$K\left(\theta_{4}\right)$ & $.3321989086 \mathrm{e}-2$ \\
\hline
\end{tabular}

Tabela 4.6. Escalares de Ricci na parametrização $\theta$

\begin{tabular}{|l|l|}
\hline & \\
\hline$K$ & $.1379085258 \mathrm{e}-4$ \\
\hline$K\left(\beta_{1}\right)$ & $.2972818042 \mathrm{e}-8$ \\
\hline$K\left(\beta_{2}\right)$ & $.1379071345 \mathrm{e}-4$ \\
\hline$K\left(\beta_{3}\right)$ & $.1379081249 \mathrm{e}-4$ \\
\hline$K\left(\beta_{4}\right)$ & $.1379081222 \mathrm{e}-4$ \\
\hline
\end{tabular}

Tabela 4.7.Escalares de Ricci na parametrização $\beta$

Portanto, notamos que o escalar de Ricci foi capaz de captar a não linearidade do modelo estatístico e sua redução diante da reparametrização mencionada nesse exemplo específico.

\section{Exemplo 2:}

Um modelo usado para reações enzimáticas é o de Michaelis-Menten:

$f=\theta_{1} x /\left(\theta_{2}+x\right)$

onde f é a taxa da reação química e $\mathrm{x}$ a concentração de substrato. De modo análogo a isomerização do n pentano, uma reparametrização conveniente seria:

$\beta_{1}=\theta_{2} / \theta_{1}$

$\beta_{2}=1 / \theta_{1}$

E o modelo seria dado por

$f=x /\left(\beta_{1}+\beta_{2} x\right)$

Para o conjunto de dados de Bates Watts (1980):

\begin{tabular}{|l|l|l|l|}
\hline $\mathrm{X}$ & $\mathrm{y}$ & $\mathrm{x}$ & $\mathrm{y}$ \\
\hline 2.000 & 0.0615 & 0.286 & 0.0129 \\
\hline 2.000 & 0.0527 & 0.286 & 0.0183 \\
\hline 0.667 & 0.0334 & 0.222 & 0.0083 \\
\hline 0.667 & 0.0258 & 0.222 & 0.0169 \\
\hline 0.400 & 0.0138 & 0.200 & 0.0129 \\
\hline 0.400 & 0.0258 & 0.200 & 0.0087 \\
\hline
\end{tabular}

Tabela 4.8. Dados simulados por Bates e Wates a partir do modelo Michaelis Menten 
Os estimadores de máxima verossimilhança são ddaos por $\theta^{+}=(0.10579,1.7007)$ e $\beta^{+}=(.062203, .5879931)$.

$\mathrm{O}$ array de curvatura dado por

$\mathrm{C}_{\theta 1}=[0,0.2930,-0.1626]$

$\mathrm{C}_{\theta 2}=[0,-0.0813,-0.7179]$

E na parametrização $\beta$ por:

$\mathrm{C}_{\beta 1}=[0,-0.038,-0.032]$

$C_{\beta 2}=[0,-0.032,-0.146]$

Notamos a redução nos termos do array de curvatura, sugerindo que a aproximação linear está mais próxima da exata na parametrização $\beta$.

O escalar de Ricci nas duas parametrizações fornece os seguintes resultados:

Parametrização $\left(\theta_{1}, \theta_{2}\right)$

$\mathrm{K}=-.2521054948 \mathrm{e} 13$

Parametrização $\left(\beta_{1}, \beta_{2}\right)$

$\mathrm{K}=-.4276742017 \mathrm{e} 13$

Notamos que a curvatura de Ricci mostrou-se eficiente em captar qual das duas parametrizações tem maior comportamento não linear nesse exemplo específico. 


\section{Capítulo 5}

\section{Estudo de Curvaturas de Distribuições Perfiladas}

\subsection{Modelo de Block Basu}

A distribuição exponencial bivariada (ACBVE) de Block e Basu com parâmetros $\lambda_{1}$ ,$\lambda_{2}$ e $\lambda_{3}$ para os tempos de vida $X$ e $Y$ tem função de densidade conjunta dada por

$F(x, y)=$

a) $f_{1}(x, y)=\lambda \lambda_{1} \lambda_{23} /\left(\lambda_{12}\right) \exp \left\{\left(-\lambda_{1} x-\lambda_{23} y\right)\right\}$

se $x<y$

b) $f_{2}(x, y)=\lambda \lambda_{2} \lambda_{13} /\left(\lambda_{12}\right) \exp \left\{\left(-\lambda_{13} x-\lambda_{2} y\right)\right\}$

se $x>y$

onde $\lambda_{12}=\lambda_{1}+\lambda_{2}, \lambda_{13}=\lambda_{1}+\lambda_{3}, \lambda_{23}=\lambda_{2}+\lambda_{3}$ e $\lambda=\lambda_{1}+\lambda_{2}+\lambda_{3}$.

Função de Verossimilhança

Os estimadores de máxima verossimilhança (EMV) para os parâmetros $\lambda_{1}, \lambda_{2}, \lambda_{3}$ são os valores de $\lambda_{1}{ }^{+}, \lambda_{2}{ }^{+}, \lambda_{3}{ }^{+}$que maximizam a função de verossimilhança

$L\left(\lambda_{1}, \lambda_{2}, \lambda_{3}\right)=\lambda_{1}{ }^{\mathrm{r}} \lambda^{\mathrm{n}} \lambda_{2}{ }^{\mathrm{n}-\mathrm{r}} \lambda_{23}{ }^{\mathrm{r}} \lambda_{13}{ }^{\mathrm{n}-\mathrm{r}} / \lambda_{12}{ }^{\mathrm{n}} \exp \left\{-\lambda_{1} \mathrm{x} \cdot-\lambda_{2} \mathrm{y} \cdot-\lambda_{3} \mathrm{Rm}\right\}$

Onde $T=\sum_{i=1}^{n} \delta_{i}, R m=\sum_{i=1}{ }^{n}\left[\delta_{i} y_{i}+\left(1-\delta_{i}\right) x_{i}\right], x .=\left(\sum_{i=1}{ }^{n} x_{i}\right) / n, y=\left(\sum_{i=1}{ }^{n} y_{i}\right) / n$ e

$\delta_{\mathrm{i}}=$

a) 1 se $X_{i}<Y_{i}$,

b) 0 se $X_{i} \geq Y_{i}$.

Sendo

$1\left(\lambda_{1}, \lambda_{2}, \lambda_{3}\right)=\ln \mathrm{L}\left(\lambda_{1}, \lambda_{2}, \lambda_{3}\right)$

$1\left(\lambda_{1}, \lambda_{2}, \lambda_{3}\right)=r \ln \lambda_{1}+(n-r) \ln \lambda_{2}+n \ln \lambda+r \ln \lambda_{23}+(n-r) \ln \lambda_{13}-n \ln \lambda_{12}-\lambda_{1}$ x. $-\lambda_{2} y .-\lambda_{3} R m$

\subsection{Reparametrizações}

a)Reparametrização Logaritmica

Considerando-se a reparametrização logarítmica para os parâmetros $\lambda_{1}, \lambda_{2}, \lambda_{3}$ tem-se

$\theta_{1}=\ln \left(\lambda_{1}\right)$

$\theta_{2}=\ln \left(\lambda_{2}\right)$

$\theta_{3}=\ln \left(\lambda_{3}\right)$

Nessa reparametrização a função de verossimilhança é dada por:

$L\left(\theta_{1}, \theta_{2}, \theta_{3}\right)=\theta_{1}{ }^{r} \theta^{n} \theta_{2}{ }^{n-r} \theta_{23}{ }^{r} \theta_{13}{ }^{n-r} \theta_{12}{ }^{n} \exp \left\{-\theta_{1} x \cdot-\theta_{2} y \cdot-\theta_{3} R m\right\}$ 
onde $\lambda_{12}=\lambda_{1}+\lambda_{2}, \lambda_{13}=\lambda_{1}+\lambda_{3}, \lambda_{23}=\lambda_{2}+\lambda_{3}$ e $\lambda=\lambda_{1}+\lambda_{2}+\lambda_{3}$.

b)Transformação de Box e Cox

Considerando-se a família de transformações de Box e Cox (1964) para reparametrizar $\left(\lambda_{1}, \lambda_{2}, \lambda_{3}\right)$ :

$$
\phi_{\mathrm{i}}=\lambda_{\mathrm{i}}\left(\varepsilon_{\mathrm{i}}\right)=
$$

a) $\left(\lambda^{\text {si }}-1\right) / \varepsilon_{\mathrm{i}}$ se $\varepsilon_{\mathrm{i}} \neq 0$

b) $\ln \left(\lambda_{i}\right)$ se $\varepsilon_{i}=0$.

para $i=1,2,3$. A função de verossimilhança é dada por $\left.\Psi_{3} \mathrm{Rm}\right\}$

$\mathrm{L}\left(\phi_{1}, \phi_{2}, \phi_{3}\right)=\Psi_{1}^{\mathrm{r}}\left(\Psi_{1}+\Psi_{2}+\Psi_{3}\right)^{\mathrm{n}} \Psi_{2}^{\mathrm{n}-\mathrm{r}}\left(\Psi_{2}+\Psi_{3}\right)^{\mathrm{r}}\left(\Psi_{1}+\Psi_{3}\right)^{\mathrm{n}-\mathrm{r}}\left(\Psi_{1}+\Psi_{2}\right)^{\mathrm{n}} \exp \left\{-\Psi_{1}\right.$ X.- $-\Psi_{2}$ y.-

onde $\Psi_{\mathrm{j}}=\left(\phi_{\mathrm{j}} \varepsilon_{\mathrm{i}}+1\right)^{1 / \varepsilon \mathrm{j}} \mathrm{j}=1,2,3$.

c)Reparametrização $\left(\lambda_{1}, \lambda_{2}, \theta_{0}\right)$

A função de confiabilidade para o modelo Block-Basu para um sistema em série no tempo $t_{0}$ é dada por

$\theta_{0}=R\left(t_{0}\right)=P\left(T>t_{0}\right)=P\left(\min (X, Y)>t_{0}\right)=\exp \left\{-\lambda t_{0}\right\}$

Dessa forma, temos

$\lambda=-\ln \left(\theta_{0}\right) / t$

$\lambda_{3}=-\ln \left(\theta_{0}\right) / t_{0}-\lambda_{1}-\lambda_{2}$

$\lambda_{23}=-\ln \left(\theta_{0}\right) / t_{0}-\lambda_{1}$

$\lambda_{13}=-\ln \left(\theta_{0}\right) / t_{0}-\lambda_{2}$

A função de verossimilhança é dada por

$L\left(\lambda_{1}, \lambda_{2}, \theta_{0}\right)=\lambda_{1}{ }^{r} \quad \lambda_{2}{ }^{n-r}\left(\lambda_{1}+\lambda_{2}\right)^{n}\left(-\ln \left(\theta_{0}\right) / t_{0}\right)^{n}\left(-\ln \left(\theta_{0}\right) / t_{0}-\lambda_{1}\right)^{r}\left(-\ln \left(\theta_{0}\right) / t_{0}-\lambda_{2}\right)^{n-r} \exp \left\{-\lambda_{1} x .-\right.$ $\left.\lambda_{2} y_{\cdot}-\left(-\ln \left(\theta_{0}\right) / t_{0}-\lambda_{1}-\lambda_{2}\right) R m\right\}$

Onde $0<\theta_{0} \leq 1$.

d)Reparametrização $\left(\lambda_{1}, \lambda_{2}, \phi_{G J}\right)$

A transformação Guerrero Johnson é dada por

$\phi=\phi_{\mathrm{GJ}}(\varepsilon)=$

a) $\left\{\left(\theta_{0} /\left(1-\theta_{0}\right)\right)^{\varepsilon}-1\right\} / \varepsilon$

se $\varepsilon \neq 0$

b) $\ln (\theta)$

se $\varepsilon=0$

A transformação inversa dada por 
$\theta=$

a) $(\phi \varepsilon+1)^{\varepsilon} /\left[1+(\phi \varepsilon+1)^{1 / \varepsilon}\right]$

se $\varepsilon \neq 0$

b) $\exp (\phi)$

se $\varepsilon=0$.

A função de verossimilhança nessa reparametrização é dada por

a) se $\varepsilon=0$

$L\left(\lambda_{1}, \lambda_{2}, \phi\right)=\left(\lambda_{1}+\lambda_{2}\right)^{-n} \lambda_{1}^{r} \lambda_{2}^{n-r} w^{n}\left(w-\lambda_{1}\right)^{r}\left(w-\lambda_{2}\right)^{n-r} \exp \left\{-\lambda_{1} x .-\lambda_{2} y .-\left(w-\lambda_{1}-\lambda_{2}\right) R m\right\}$

onde $w=-\ln \left(\theta_{0}\right) / t_{0}$

b)se $\varepsilon \neq 0$

$L\left(\lambda_{1}, \lambda_{2}, \phi\right)=\left(\lambda_{1}+\lambda_{2}\right)^{-n} \lambda_{1}^{r}\left(-p_{1}\right)^{n} \lambda_{2}^{n-r}\left(-p_{1}-\lambda_{1}\right)^{r}\left(-p_{1}-\lambda_{2}\right)^{n-r} \exp \left\{-\lambda_{1}\right.$ x. $-\lambda_{2}$ y. $\left.-\left(-p_{1}-\lambda_{1}-\lambda_{2}\right) R m\right\}$ onde

$\mathrm{P}=\ln \left[(\varepsilon \phi+1)^{1 / \varepsilon} /\left((\varepsilon \phi+1)^{1 / \varepsilon}+1\right)\right] / \mathrm{t}_{0}$

Um critério que pode ser usado para calcular $\varepsilon$ é o valor de $\varepsilon$ que minimiza a medida de Kass Slate:

$\mathrm{STD}=\sum_{\mathrm{ijklmn}} b_{\mathrm{il}} b_{\mathrm{jm}} b_{\mathrm{kn}} \mathrm{d}_{\mathrm{ijk}} \mathrm{d}_{\mathrm{lmn}}$

Onde $b_{i j}$ são os elementos da inversa da matriz de informação de Fisher observada, $\mathrm{d}_{\mathrm{ijk}}=\partial \mathrm{l}\left(\theta^{+}\right) / \partial \theta_{\mathrm{i}} \partial \theta_{\mathrm{j}} \partial \theta_{\mathrm{k}}$, para um parâmetro $\mathrm{m}$ dimensional $\theta$ e a soma tomada sobre todos os indices.

\section{e)Reparametrização Natural}

Se os tempos de vida de um sistema de dois componentes seguem uma distribuição Block Basu, os tempos médios de vida $\mu_{1}=E(X)$ e $\mu_{2}=E(Y)$ desses componentes são dados por:

$$
\begin{aligned}
& \mu_{1}=1 / \lambda_{13}+\lambda_{2} \lambda_{3} /\left(\lambda \lambda_{12} \lambda_{23}\right) \\
& \mu_{2}=1 / \lambda_{23}+\lambda_{1} \lambda_{3} /\left(\lambda \lambda_{12} \lambda_{23}\right)
\end{aligned}
$$

No caso de um sistema composto por dois componentes, ligados em série, o sistema funciona se os dois componentes funcionam simultaneamente. Se tais componentes têm distribuição de vida com função

de densidade Block Basu, a função de confiabilidade do sistema no tempo to é dada por:

$$
R_{S}\left(t_{0}\right)=P\left[\min (X, Y)>t_{0}\right]=\exp \left(-\lambda t_{0}\right)
$$

É comum ocorrer na prática a existência de informações sobre prováveis tempos médios de vida de um componente $1\left(\mu_{1}\right)$, do componente $2\left(\mu_{2}\right)$ e sobre a confiabilidade do sistema $\left(\theta_{0}\right)$. Essa informação pode ser utilizada se for usada uma reparametrização que possa expressar $\lambda_{1}, \lambda_{2}, \lambda_{3}$ como função de $\mu_{1}, \mu_{2}, \theta_{0}$.

Desse modo, temos

$$
\begin{aligned}
& \mu_{1}=1 / \lambda_{13}+\lambda_{2} \lambda_{3} /\left(\lambda_{12} \lambda_{13} \lambda\right) \\
& \mu_{2}=1 / \lambda_{23}+\lambda_{1} \lambda_{3} /\left(\lambda_{12} \lambda_{23} \lambda\right)
\end{aligned}
$$


$\theta_{0}=\exp \left\{\lambda t_{0}\right\}$

As figuras 5.1 a 5.9 apresentam alguns t-plots, verossimilhanças profiles e contornos de verossimilhanças profiles. Comparando-se tais gráficos, identificamos que em algumas parametrizações há maior comportamento normal nas distribuições perfiladas do que em outras. 

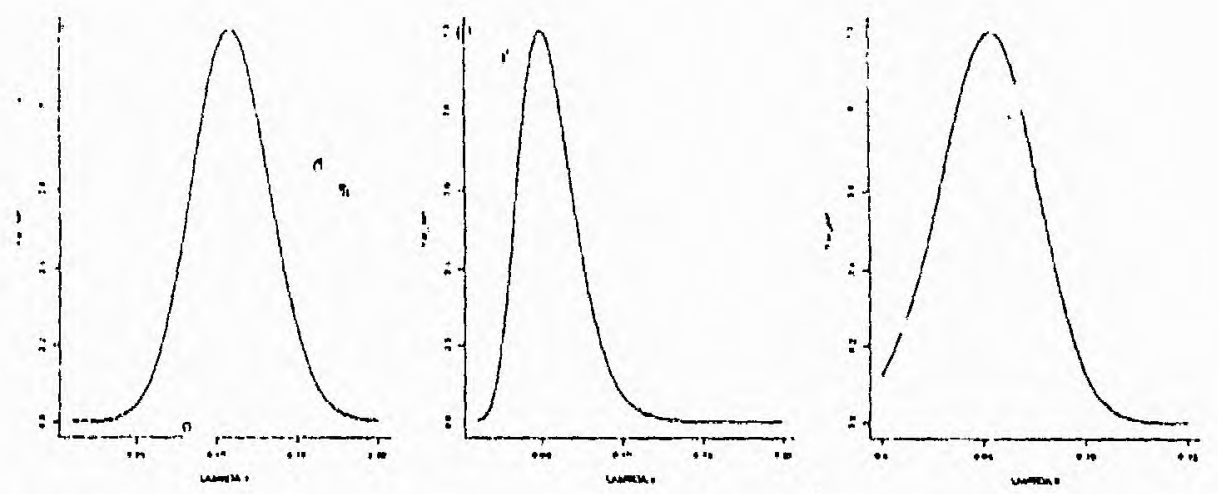

Figura 5.1. Grálico da verossimill
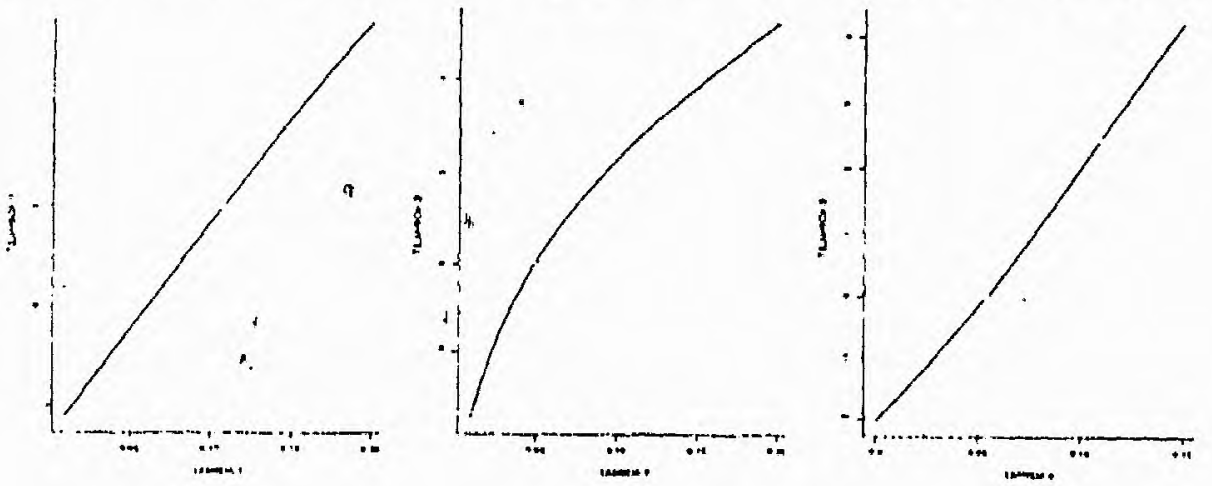

Figura 5.2. "t-plot" para $\lambda_{1}, \lambda_{2}$ e $\lambda_{3}$ respectivamente"
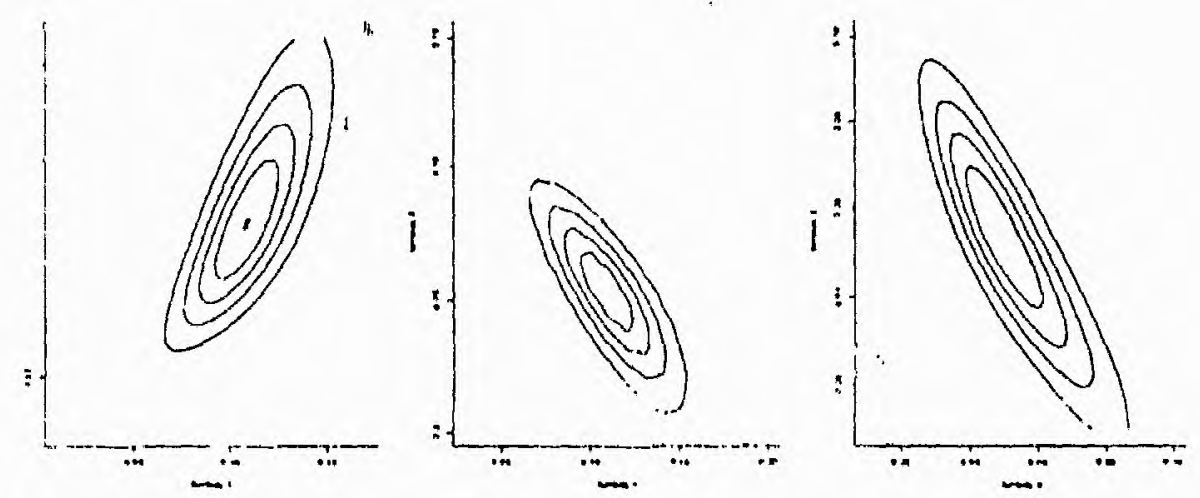

Figura 5.3. Contorno da verussinnilhanģa perfilata para. $\left(\lambda_{1}, \lambda_{2}\right)_{2},\left(\lambda_{1}, \lambda_{3}\right),\left(\lambda_{2}, \lambda_{3}\right)$. respectivamente 

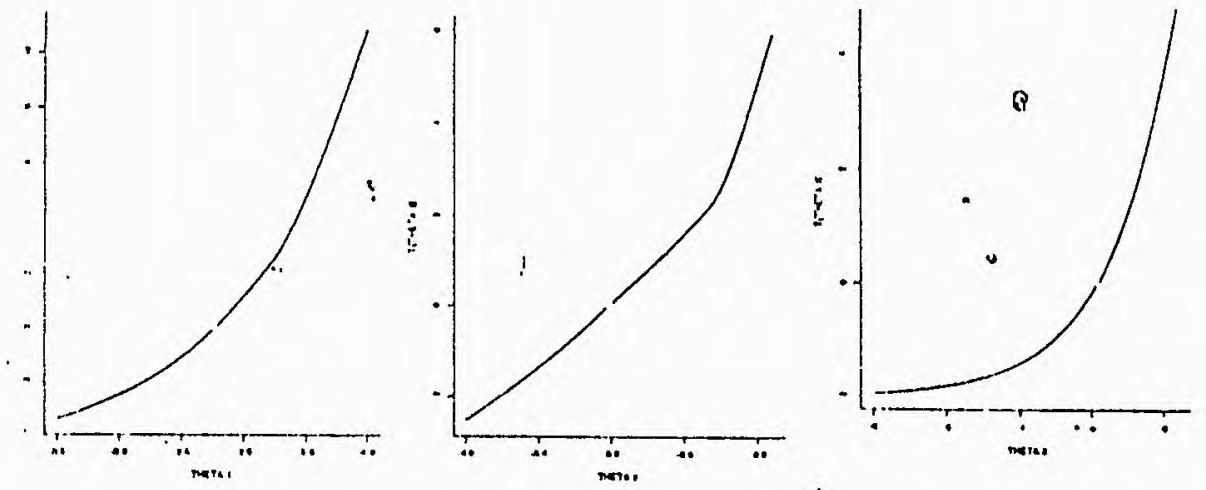

Figura 5.5. "t-plot" para $\theta_{1}, \theta_{2}$ e $\theta_{3}$ respectivamente
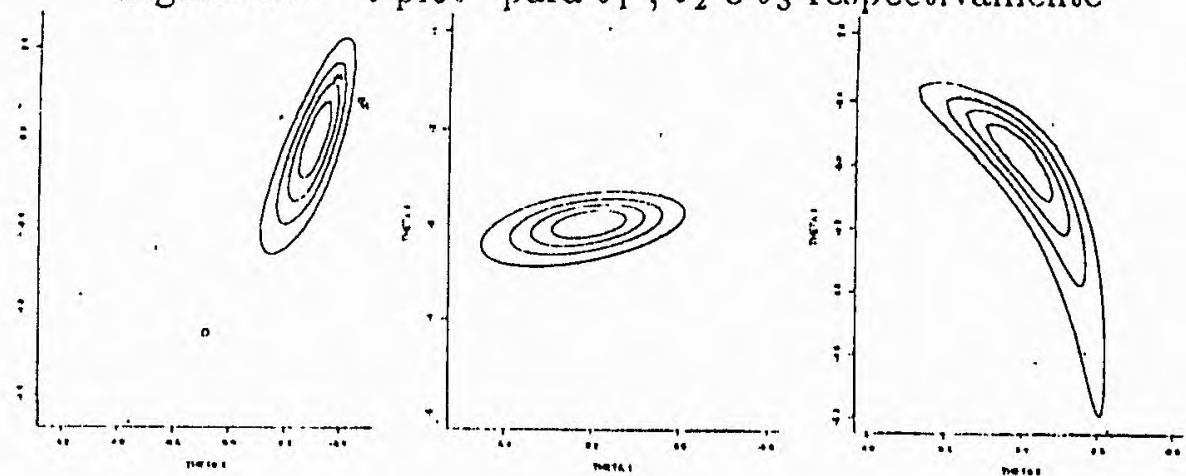

Figura 5.6. Coptorno da verossimilhança perfilada para $\left(\theta_{1}, 0_{2}\right),\left(\theta_{1}, 0_{3}\right),\left(0_{2}, 0_{3}\right)$ respectivamente 

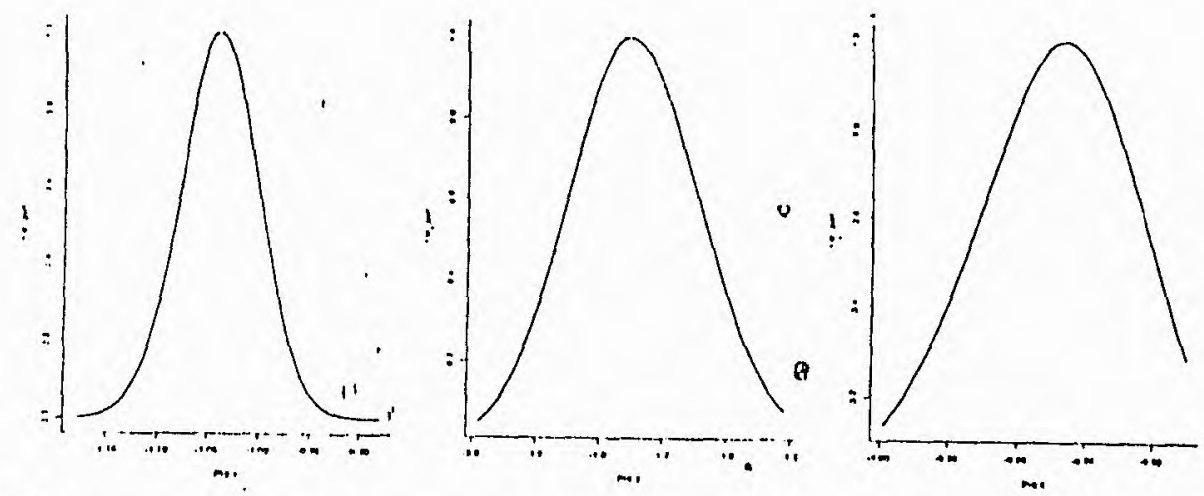

Figura 5.7. Ciráfico da verossimilhauga perfilada para $\phi_{1}, \phi_{2}$ e $\phi_{3}$ respectivamente.
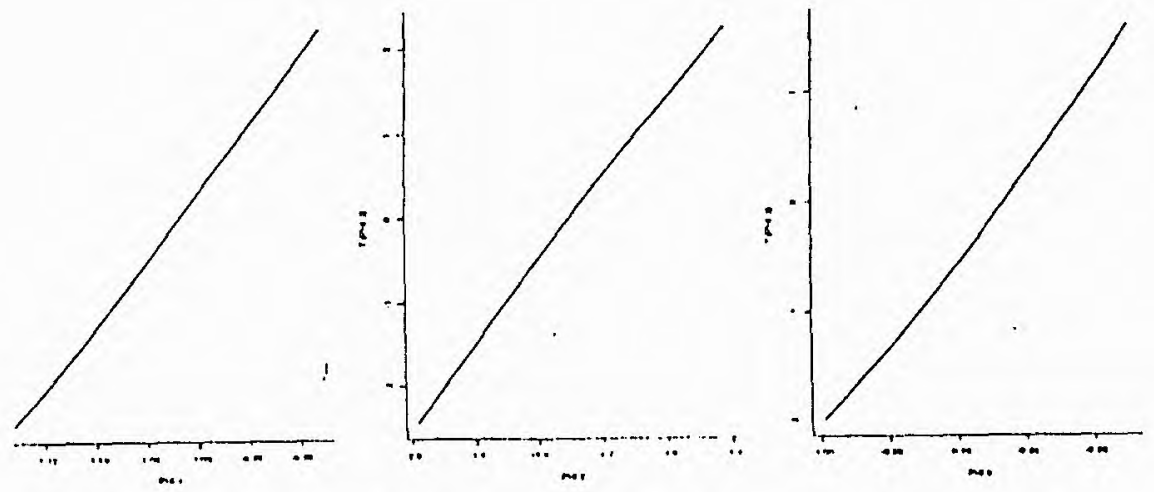

Figura 5.8. "1,-plot" para $\phi_{1}, \phi_{2}$ c $\phi_{3}$ respectivanente
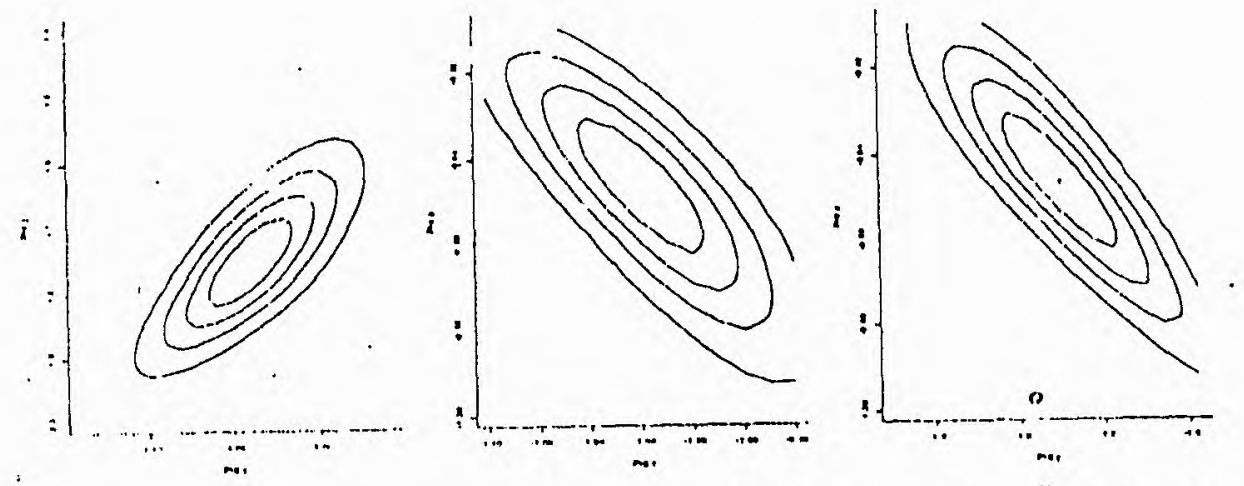

Figura 5.9. Cintorno da verossimilhança perfilada para $\left(\phi_{1}, \phi_{2}^{\prime \prime}\right),\left(\phi_{1}, \phi_{3}\right),\left(\phi_{2}, \phi_{3}\right)$ iespectivamentc 
l'abela 5 1: Dados de tempe de vida bivariados

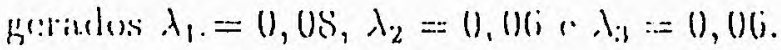

\begin{tabular}{|c|c|c|c|c|c|}
\hline$x$ & 1 & $x$ & 1 & $\therefore$ & $y$ \\
\hline $0,(11,1, !)$ & 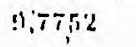 & $0,322: 1$ & $\mid 11 . \therefore 1: 1.1$ & $17.1 \times 8.7$ & 3.9211 \\
\hline (1).11:10iot & $\because 1,7 ! 121$ & m,usontiti & 10,51077 & $\cdot 1.720$ & $11,1)(1) 2$ \\
\hline U,ulisus, & $!, 818 !$ & $1,0(1,15,1$ & $1(1 .(i))^{\prime}$ & I.filitift & 11,1860 \\
\hline $0,118: 125$ & 01.83316 & 1.033314 & III. & $1,4(1) \cdot 1$ & 11,31000 \\
\hline $0,11: 1,12$ & 0,8570 & $1.0733 t i$ & IU.(iT:I:I & $2.2+1.13$ & $11,(\mathrm{i}) 2 !)$ \\
\hline 1).1:3787 & 9,8775 & $1,101,12$ & 111.6i:)72 & $2.1: 1 ? k$ & 12,01372 \\
\hline (1,1,1:R7 & 0,8412 & 1,11178 & $\mid\left(1, \pi()_{i} \mid\right.$ & $: 1,112,1$ & $12,4(i, 3)$ \\
\hline 0.2010018 & $9,90: 37.1$ & $1,1,1278$ & $10.7: 12 \mu$ & $a^{1}, 1 ! 27 s$ & 12,8087 \\
\hline $11,2: 1578$ & 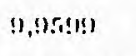 & 1,17 sliti & |ll,iti:I) & $: 1.8 .18: 1$ & $1: 1,1215$ \\
\hline $0,2 \mu$ II $_{1}$ & $10.001 .10)$ & 1,20725 & $\mid 0,734.1$ & $\{.31112$ & $1: 3,5,13010$ \\
\hline 0,$11271 ;: 3$ & $110,0377: 1$ & $1,2.5+101$ & 111.82844 & $1,75.32$ & 13,94667 \\
\hline 0.36 .21$)$ & 10.0580 & $8,0881 \cdot 1$ & $0.02: 12$ & 5,31500 & $1.1,161.1$ \\
\hline $0.377 !)$ & 10.0798 & 8,$276 ; 2$ & $0,2: 1311$ & $\therefore,(i t) \cdot 11$ & 14,8105 \\
\hline $0,1: 1$ ) & 10,0058 & $8.527 !$ & $0, \therefore, 1, n: 3$ & (i.15)8 & $15,21\} 1$ \\
\hline $0.410 ! 11.1$ & 10,10602 & 8,7821 & 0.77197 & (i,7u:s: & $15,75,22$ \\
\hline 0.41137 .1 & 111,1101 & $0,0.515$ & 1,116!: & 7.5:14: & $16,5.760 .4$ \\
\hline$(1,12769 !)$ & $1(1.121 !)$ & $0,2105,2$ & $1.3: 116$ & M.H.H. & 46,9587 \\
\hline $0,+1100$ & 10,1332 & 0,5615 & I.fillid & $x,\{: 75\}$, & $17,50($ i!) \\
\hline U, Hititin & $(1), 15.14)$ & $0,87: 17$ & $1,057: 1$ & $\because \because \cdot 98: 1$ & 18,3567 \\
\hline $0.47: 38.3$ & $10.1(i) 0$ & $10,18: 31$ & 2,24161 & lo. Iriciri & 10,2020 \\
\hline $0.5+1): 1.1: 1$ & Iti,Isfil & $101,42 \cdot 131$ & $2.535 \div 8$ & $11.1+1.00$ & $10.9 \cdot 158$ \\
\hline $0,5187: 3$ & 10.1901 & $10,882 \cdot 1$ & 3,11241 & 191213 & 20,8825 \\
\hline $0,597,6,7$ & $10,21: 15$ & 11,3216 & 3,4897 & $18,60.37$ & $11.0(i) 5$ \\
\hline (1).51ing & 10.2372 & $11,7: 383$ & $3.92 ! 9 !)$ & 10.33300 & 11.8160 \\
\hline $0,6311: 36$ & 10,2778 & $12,2: 11 ; 8$ & 1,15522 & $20.1 \cdot 18.3$ & 12,6555 \\
\hline $0,0,102:\}$ & 10,3075 & 12,7201 & $4,3(1 ; 37$ & $22.585,3$ & 17,1477 \\
\hline O,fisprici & 10,31316 & $13,2: 17: 3$ & $5.505,4$ & 13..3к2! & 22,09881 \\
\hline $0,700.17$ & 10,3614 & 14,$06 ; 23$ & (6.36)7 & $1 \cdot 1.1032$ & 23,0855 \\
\hline $0,7 \cdot 1352$ & $10,395.5$ & 15,0128 & $7, \therefore, 3 \pi 7$ & 15.15 .17 & $2: 3,81: 37$ \\
\hline $0.311(i)$ & 10.1186 & $15,35,711$ & $7.715: 3$ & $2: 1,06: 7$ & $31,56,609$ \\
\hline $0.85 .31 !$ & $10,18.12$ & 15,4718 & $7,8: 3.1$ & 31.8155 & 24,5140 \\
\hline 0.8785 .1 & 10,5050 & $15,8001 i$ & 8.26 isk & $2, \pi, \pi 304 i$ & 33,9380 \\
\hline \multirow[t]{2}{*}{ 7.:31313 } & $10,5 ; 3,16)$ & Ifo,5lik) & $8,: 1702$ & $: 12.161 .1 .1$ & .0 .5758 \\
\hline & & $17.15, K M$ & :1.5, & & \\
\hline
\end{tabular}




\subsection{Relação entre a curvatura global do modelo a três parâmetros} e as curvaturas das distribuições perfiladas a dois parâmetros

Na parametrização $\left(\lambda_{1}, \lambda_{2}, \lambda_{3}\right)$ temos o logaritmo da função de verossimilhança dado por (dados da tabela 5.1)

$1\left(\lambda_{1}, \lambda_{2}, \lambda_{3}\right)=71 \ln \left(\lambda_{1}\right)+29 \ln \left(\lambda_{2}\right)+100 \ln \left(\lambda_{1}+\lambda_{2}+\lambda_{3}\right)+71 \ln \left(\lambda_{2}+\lambda_{3}\right)+29 \ln \left(\lambda_{1}+\lambda_{2}\right)-100$ $\ln \left(\lambda_{1}+\lambda_{2}\right)-674,45 \lambda_{1}-1188,88 \lambda_{2}-1343,65 \lambda_{3}$

Os estimadores de máxima verossimilhança são dados por $\left(\lambda_{1}{ }^{+}\right.$ $\left., \lambda_{2}{ }^{+}, \lambda_{3}{ }^{+}\right)=(0,10782 ; 0,04896 ; 0,05396)$.

Nessa parametrização temos os seguintes valores para o tensor de Ricci:

$$
\begin{aligned}
& \mathrm{R}_{11}=.5915632965 \mathrm{e}-3 \\
& \mathrm{R}_{12}=.5993243700 \mathrm{e}-3 \\
& \mathrm{R}_{13}=-.7368063539 \mathrm{e}-3 \\
& \mathrm{R}_{22}=.6420659280 \mathrm{e}-3 \\
& \mathrm{R}_{23}=-.7740522449 \mathrm{e}-3 \\
& \mathrm{R}_{33}=.9534731004 \mathrm{e}-3
\end{aligned}
$$

Desse modo, o escalar de Ricci é dado por

$$
\mathrm{K}=\mathrm{g}^{\mathrm{ii}} \mathrm{g}^{\mathrm{ij}} \mathrm{R}_{\mathrm{ij}}=-.2673660760 \mathrm{e}-5
$$

Essa é a curvatura escalar da distribuição conjunta, a três parâmetros. Ela pode ser decomposta em duas somas:

$$
\begin{aligned}
& \mathrm{K}=\mathrm{K}_{\mathrm{a}}+\mathrm{K}_{\mathrm{b}} \\
& \mathrm{K}_{\mathrm{a}}=\mathrm{g}^{11} \mathrm{~g}^{22} \mathrm{R}_{12}+\mathrm{g}^{11} \mathrm{~g}^{33} \mathrm{R}_{13}+\mathrm{g}^{22} \mathrm{~g}^{33} \mathrm{R}_{23} \\
& \mathrm{~K}_{\mathrm{b}}=\mathrm{g}^{11} \mathrm{~g}^{11} \mathrm{R}_{11}+\mathrm{g}^{22} \mathrm{~g}^{22} \mathrm{R}_{22}+\mathrm{g}^{33} \mathrm{~g}^{33} \mathrm{R}_{33}
\end{aligned}
$$

A primeira soma pode ser entendida como soma de três escalares de Ricci:

$$
\begin{aligned}
& \mathrm{K}_{\mathrm{a}}=\mathrm{K}_{\mathrm{ab}}+\mathrm{K}_{\mathrm{ac}}+\mathrm{K}_{\mathrm{bc}} \\
& \mathrm{K}_{\mathrm{ab}}=\mathrm{g}^{11} \mathrm{~g}^{22} \mathrm{R}_{12} \\
& \mathrm{~K}_{\mathrm{ac}}=\mathrm{g}^{11} \mathrm{~g}^{33} \mathrm{R}_{13} \\
& \mathrm{~K}_{\mathrm{bc}}=\mathrm{g}^{22} \mathrm{~g}^{33} \mathrm{R}_{23}
\end{aligned}
$$

Essas parcelas $\left(\mathrm{K}_{\mathrm{ab}}, \mathrm{K}_{\mathrm{ac}}, \mathrm{K}_{\mathrm{bc}}\right)$ seriam as curvaturas escalares de Ricci das distribuições perfiladas, mas não são porque quando tomamos as distribuições perfiladas e calculamos os seus respectivos $\mathrm{g}^{\mathrm{ij}}$ e $\mathrm{R}_{\mathrm{ij}}$, estes não são os mesmos da distribuição conjunta.

Vamos ilustrar tal fato na parametrização $\left(\lambda_{1}, \lambda_{2}, \lambda_{3}\right)$ :

$l_{\lambda 3}$ é a verossimilhança quando substituimos $\lambda_{3}$ por seu estimador de máxima verossimilhança $\lambda_{3}{ }^{+}$:

$1_{\lambda 3}\left(\lambda_{1}, \lambda_{2}\right)=71 \ln \left(\lambda_{1}\right)+29 \ln \left(\lambda_{2}\right)+100 \ln \left(\lambda_{1}+\lambda_{2}+0,05396\right)+71 \ln \left(\lambda_{2}+0,05396\right)+29$ $\ln \left(\lambda_{1}+\lambda_{2}\right)-100 \ln \left(\lambda_{1}+\lambda_{2}\right)-674,45 \lambda_{1}-1188,88 \lambda_{2}-1343,65(0,05396)$

$l_{\lambda 2}$ é a verossimilhança quando substituímos $\lambda_{3}$ por seu estimador de máxima verossimilhança $\lambda_{2}{ }^{+}$: 
$\mathrm{l}_{\lambda 2}\left(\lambda_{1}, \lambda_{3}\right)=71 \ln \left(\lambda_{1}\right)+29 \ln (0,04896)+100 \ln \left(\lambda_{1}+0,04896+\lambda_{3}\right)+71 \ln \left(0,04896+\lambda_{3}\right)+29$ $\ln \left(\lambda_{1}+0,04896\right)-100 \ln \left(\lambda_{1}+0,04896\right)-674,45 \lambda_{1}-1188,88(0,04896)-1343,65 \lambda_{3}$

$l_{\lambda 1}$ é a verossimilhança quando substituímos $\lambda_{3}$ por seu estimador de máxima verossimilhança $\lambda_{1}^{+}$:

$1_{\lambda 1}\left(\lambda_{2}, \lambda_{3}\right)=71 \ln (0,10782)+29 \ln \left(\lambda_{2}\right)+100 \ln \left(0,10782+\lambda_{2}+\lambda_{3}\right)+71 \ln \left(\lambda_{2}+\lambda_{3}\right)+29$ $\ln \left(0,10782+\lambda_{2}\right)-100 \ln \left(0,10782+\lambda_{2}\right)-674,45(0,10782)-1188,88 \lambda_{2}-1343,65 \lambda_{3}$

Vamos apresentar as quantidades de interesse:

a) Valores para a perfilada $\mathrm{h}_{\lambda_{3}}$

\begin{tabular}{|l|}
\hline$-.1921423266 \mathrm{e}-3$ \\
\hline$-.2055206284 \mathrm{e}-4$ \\
\hline$-.6107653171 \mathrm{e}-4$ \\
\hline
\end{tabular}

Tabela 5.1. Valores da matriz inversa de Fisher

\begin{tabular}{|l|}
\hline $.3173815885 \mathrm{e}-4$ \\
\hline $.3353854394 \mathrm{e}-5$ \\
\hline $.1337959748 \mathrm{e}-4$ \\
\hline
\end{tabular}

Tabela 5.2.Valores do tensor de Ricci

Escalar de Ricci $=.7053280345 \mathrm{e}-8$

b) Valores para a perfilada $\mathrm{l}_{\lambda 2}$

\begin{tabular}{|l|}
\hline$-.2338060326 \mathrm{e}-3$ \\
\hline $.7806358927 \mathrm{e}-4$ \\
\hline$-.1254425244 \mathrm{e}-3$ \\
\hline
\end{tabular}

Tabela 5.3.Valores da matriz inversa de Fisher

\begin{tabular}{|l|}
\hline $.6649638296 \mathrm{e}-4$ \\
\hline$-.2608180803 \mathrm{e}-4$ \\
\hline $.2165067254 \mathrm{e}-4$ \\
\hline
\end{tabular}

Tabela 5.4.Valores do tensor de Ricci

Escalar de Ricci $=-.2233524960 \mathrm{e}-7$

c) Valores para a perfilada $l_{\lambda 1}$

\begin{tabular}{|l|}
\hline$-.1109175635 \mathrm{e}-3$ \\
\hline$-.9870403551 \mathrm{e}-4$ \\
\hline$-.1872138918 \mathrm{e}-3$ \\
\hline
\end{tabular}

Tabela 5.5.Valores da matriz inversa de Fisher

\begin{tabular}{|l|}
\hline $.8098412619 \mathrm{e}-4$ \\
\hline$-.7199688321 \mathrm{e}-4$ \\
\hline $.7310508388 \mathrm{e}-4$ \\
\hline
\end{tabular}

Tabela 5.6. Valores do tensor de Ricci

Escalar de Ricci $=-.3688161506 \mathrm{e}-7$ 
Calculando-se os respectivos escalares de Ricci da distribuição conjunta temos (Segunda coluna da tabela):

\begin{tabular}{|l|l|l|}
\hline Parâmetros envolvidos & K parciais & $\begin{array}{l}\text { K nas distribuições } \\
\text { perfiladas }\end{array}$ \\
\hline$\left(\lambda_{1}, \lambda_{2}\right)$ & $.9670525459 \mathrm{e}-10$ & $-.7053280345 \mathrm{e}-8$ \\
\hline$\left(\lambda_{1}, \lambda_{3}\right)$ & $-.2441810266 \mathrm{e}-9$ & $-.2233524960 \mathrm{e}-7$ \\
\hline$\left(\lambda_{2}, \lambda_{3}\right)$ & $-.1216951965 \mathrm{e}-9$ & $-.3688161506 \mathrm{e}-7$ \\
\hline
\end{tabular}

Tabela 5.7.comparação dos escalares de Ricci parciais e das perfiladas

Conforme vimos, os valores dos escalares de Ricci na conjunta (escalares de Ricci parciais) e nas perfiladas são distintos.

Vamos mostrar, somente a título de ilustração, os valores do escalar de Ricci diante de duas reparametrizações onde dois parâmetros ficam fixos $\lambda_{1}$ e $\lambda_{2}$ :

1) $\left(\lambda_{1}, \lambda_{2}, \theta_{0}\right)$

2) $\left(\lambda_{1}, \lambda_{2}, \phi \mathrm{GJ}\right)$

Os valores estão nas tabelas 5.9-5.12 e 5.14-5.17. As tabelas 5.8 e 5.13 mostram os tempos de vida onde foram calculados os tensores, estimativas de máxima verossimilhança de $\theta_{0}$, medidas de Kass Slate e intervalos de confiança.

As curvaturas parciais $K_{\lambda j}, j=1,2,3$, referem-se a soma de todas as parcelas que não envolvem o parâmetro $\lambda_{j}$ na curvatura de Ricci da distribuição conjunta. As curvaturas das distribuições perfiladas referem-se as curvaturas de Ricci da respectiva distribuição perfilada. 
Reparametrização $\left(\lambda_{1}, \lambda_{2}, \theta_{0}\right)$

\begin{tabular}{|l|l|l|l|}
\hline To & $\theta_{0}{ }^{+}$ & STD & $\begin{array}{l}\text { Intervalo de } \\
\text { Confiança 95\% }\end{array}$ \\
\hline 1 & 0.8100 & 0.7236 & $(0.7780 ; 0.8420)$ \\
\hline 2 & 0.6561 & 0.6602 & $(0.6042 ; 0.7079)$ \\
\hline 3 & 0.5314 & 0.6066 & $(0.4684 ; 0.5944)$ \\
\hline 5 & 0.3486 & 0.5288 & $(0.2797 ; 0.4176)$ \\
\hline 10 & 0.1216 & 0.5069 & $(0.0735 ; 0.1626)$ \\
\hline 20 & 0.0148 & 1.2021 & $(0.0031 ; 0.0266)$ \\
\hline 30 & 0.0018 & 2.8827 & $(-3 \mathrm{e}-4 ; 39 \mathrm{e}-4)$ \\
\hline
\end{tabular}

Tabela 5.8. Alguns valores de inferência na reparametrização $\left(\lambda_{1}, \lambda_{2}, \theta_{0}\right)$

\begin{tabular}{|l|l|}
\hline $\mathrm{T}_{0}$ & Conjunta \\
\hline 1 & $-.8767565986 \mathrm{e}-1$ \\
\hline 2 & $-.1687650270 \mathrm{e}-5$ \\
\hline 3 & $-.1650335023 \mathrm{e}-7$ \\
\hline 5 & $.7962901282 \mathrm{e}-3$ \\
\hline 10 & $.1963830022 \mathrm{e}-4$ \\
\hline 20 & $.2040754278 \mathrm{e}-6$ \\
\hline 30 & $.1509427179 \mathrm{e}-7$ \\
\hline
\end{tabular}

Tabela 5.9.escalar de Ricci na reparametrização $\left(\lambda_{1}, \lambda_{2}, \theta_{0}\right)$

\begin{tabular}{|l|l|}
\hline $\mathrm{K}_{\mathrm{bc}}$ & Perfilada 1 \\
\hline$-.7345043541 \mathrm{e}-2$ & $-.2077472958 \mathrm{e}-6$ \\
\hline$-.6781290043 \mathrm{e}-6$ & $-.2558598349 \mathrm{e}-7$ \\
\hline$-.1841490597 \mathrm{e}-7$ & $-.8783679222 \mathrm{e}-9$ \\
\hline$-.9578034494 \mathrm{e}-3$ & $-.1174924112 \mathrm{e}-7$ \\
\hline$-.2225130373 \mathrm{e}-4$ & $-.1216742657 \mathrm{e}-7$ \\
\hline $.3187636489 \mathrm{e}-6$ & $.1342488964 \mathrm{e}-7$ \\
\hline $.2759583085 \mathrm{e}-7$ & $.3083676754 \mathrm{e}-7$ \\
\hline
\end{tabular}

Tabela 5.10.comparação entre escalar de Ricci parcial e escalar da perfilada

\begin{tabular}{|l|l|}
\hline $\mathrm{K}_{\mathrm{ac}}$ & Perfilada 2 \\
\hline$-.5445361441 \mathrm{e}-1$ & $-.4419315040 \mathrm{e}-6$ \\
\hline$-.1081091940 \mathrm{e}-5$ & $-.7166679156 \mathrm{e}-7$ \\
\hline$-.1832451784 \mathrm{e}-7$ & $-.1094960918 \mathrm{e}-8$ \\
\hline$-.8579062598 \mathrm{e}-3$ & $-.6351920641 \mathrm{e}-6$ \\
\hline $.1999156938 \mathrm{e}-4$ & $-.2447689943 \mathrm{e}-6$ \\
\hline $.1765264081 \mathrm{e}-6$ & $.9100065862 \mathrm{e}-7$ \\
\hline $.3155017145 \mathrm{e}-7$ & $.1998848649 \mathrm{e}-7$ \\
\hline
\end{tabular}

Tabela 5.11.comparação entre escalar de Ricci parcial e escalar da perfilada

\begin{tabular}{|l|l|}
\hline $\mathrm{K}_{\mathrm{ab}}$ & Perfilada 3 \\
\hline$-.8660395681 \mathrm{e}-1$ & -2.849732318 \\
\hline$-.1507888625 \mathrm{e}-5$ & -.7953414394 \\
\hline$-.7491029882 \mathrm{e}-8$ & $-.6279599306 \mathrm{e}-2$ \\
\hline$-.2238016340 \mathrm{e}-3$ & $-.8359133062 \mathrm{e}-4$ \\
\hline$-.9964100894 \mathrm{e}-5$ & $-.3591969650 \mathrm{e}-6$ \\
\hline $.2041456567 \mathrm{e}-6$ & $-.4713749048 \mathrm{e}-8$ \\
\hline $.1367347377 \mathrm{e}-7$ & $-.7052514140 \mathrm{e}-9$ \\
\hline
\end{tabular}

Tabela 5.12.comparação entre escalar de Ricci parcial e escalar da perfilada 


\begin{tabular}{|l|l|l|l|l|}
\hline To & $\varepsilon$ & $\theta$ & STD & $\begin{array}{l}\text { Intervalo de } \\
\text { Confianca 95\% }\end{array}$ \\
\hline 1 & 0.6 & 0.8100 & 0.4875 & $(0.7734 ; 0.8383)$ \\
\hline 2 & 0.8 & 0.6561 & 0.4874 & $(0.5967 ; 0.7020)$ \\
\hline 3 & 0.8 & 0.5314 & 0.4878 & $(0.4608 ; 0.5881)$ \\
\hline 5 & 0.6 & 0.3486 & 0.4883 & $(0.2769 ; 0.4143)$ \\
\hline 10 & 0.6 & 0.1216 & 0.4865 & $(0.0757 ; 0.1709)$ \\
\hline 20 & 0.4 & 0.0148 & 0.4872 & $(0.0057 ; 0.0292)$ \\
\hline 30 & 0.2 & 0.0018 & 0.5035 & $(0.0005 ; 0.0052)$ \\
\hline
\end{tabular}

Tabela 5.13. Alguns valores de inferência na reparametrização $\left(\lambda_{1}, \lambda_{2}, \phi_{\mathrm{GJ}}\right)$

\begin{tabular}{|l|l|}
\hline $\mathrm{T}_{0}$ & Conjunta \\
\hline 1 & -2.723203972 \\
\hline 2 & -.9469858518 \\
\hline 3 & $-.7381326058 \mathrm{e}-2$ \\
\hline 5 & $-.1183526651 \mathrm{e}-3$ \\
\hline 10 & $-.1966468859 \mathrm{e}-5$ \\
\hline 20 & $-.5079163613 \mathrm{e}-5$ \\
\hline 30 & $-.3538485428 \mathrm{e}-5$ \\
\hline
\end{tabular}

Tabela 5.14. escalar de Ricci na reparametrização $\left(\lambda_{1}, \lambda_{2}, \phi_{\mathrm{GJ}}\right)$

\begin{tabular}{|l|l|}
\hline $\mathrm{K}_{\lambda_{1}}$ & Perfilada 1 \\
\hline$-.9937241257 \mathrm{e}-1$ & $-.2454797302 \mathrm{e}-6$ \\
\hline$-.4375801108 \mathrm{e}-1$ & $-.2319425931 \mathrm{e}-6$ \\
\hline$-.3976253922 \mathrm{e}-3$ & $-.1388124024 \mathrm{e}-7$ \\
\hline$-.1060339479 \mathrm{e}-4$ & $-.1489676683 \mathrm{e}-7$ \\
\hline$-.1435956562 \mathrm{e}-5$ & $-.2929460184 \mathrm{e}-7$ \\
\hline$-.5337806884 \mathrm{e}-5$ & $-.1523235370 \mathrm{e}-6$ \\
\hline$-.3560069258 \mathrm{e}-5$ & $-.3410564509 \mathrm{e}-7$ \\
\hline
\end{tabular}

Tabela 5.15.comparação entre escalar de Ricci parcial e escalar da perfilada

\begin{tabular}{|l|l|}
\hline $\mathrm{K}_{\lambda 2}$ & Perfilada 2 \\
\hline-1.779043408 & $-.2454797302 \mathrm{e}-6$ \\
\hline-.5848737888 & $-.2319425931 \mathrm{e}-6$ \\
\hline$-.4400435706 \mathrm{e}-2$ & $-.2094705694 \mathrm{e}-6$ \\
\hline$-.6729789922 \mathrm{e}-4$ & $-.1510971248 \mathrm{e}-6$ \\
\hline$-.1530134273 \mathrm{e}-5$ & $-.9684248551 \mathrm{e}-7$ \\
\hline$-.5314458234 \mathrm{e}-5$ & $-.1368829964 \mathrm{e}-3$ \\
\hline$-.3563566222 \mathrm{e}-5$ & $-.1570951684 \mathrm{e}-5$ \\
\hline
\end{tabular}

Tabela 5.16.comparação entre escalar de Ricci parcial e escalar da perfilada

\begin{tabular}{|l|l|}
\hline $\mathrm{K}_{\lambda 3}$ & Perfilada 3 \\
\hline-2.723190339 & $-.1219155189 \mathrm{e}-7$ \\
\hline-.9469695788 & $-.9807794901 \mathrm{e}-8$ \\
\hline$-.7380572789 \mathrm{e}-2$ & $-.4259987381 \mathrm{e}-8$ \\
\hline$-.6729789922 \mathrm{e}-4$ & $-.1015853319 \mathrm{e}-8$ \\
\hline$-.1504708210 \mathrm{e}-5$ & $-.1281028227 \mathrm{e}-9$ \\
\hline$-.4860682132 \mathrm{e}-6$ & $-.6526115609 \mathrm{e}-9$ \\
\hline$-.5388246336 \mathrm{e}-7$ & $-.1241671234 \mathrm{e}-8$ \\
\hline
\end{tabular}

Tabela 5.17.comparação entre escalar de Ricci parcial e escalar da perfilada 
Notamos que a parametrização $\left(\lambda_{1}, \lambda_{2}, \phi_{\mathrm{gj}}\right)$ mostra-se com menores medidas de Kass Slate do que a parametrização $\left(\lambda_{1}, \lambda_{2}, \theta_{0}\right)$. A curvatura de Ricci da distribuição conjunta segue o mesmo comportamento qualitativo.

Conforme discutido anteriormente não é possível uma relação algébrica simples entre as curvaturas das distribuições perfiladas e da distribuição conjunta. Notamos que elas não são iguais. As tabelas acima estão exercendo um papel apenas ilustrativo.

No caso de distribuições conjuntas com dois parâmetros e distribuições perfiladas com um parâmetro não podemos aplicar as fórmulas tensoriais para as distribuições perfiladas, pois o escalar de Ricci não existe em dimensão um. O que chega mais próximo de uma relação matematicamente simples entre curvaturas seria um resultado das curvaturas geométricas:

As curvaturas principais das distribuições perfiladas, que são distribuições unidimensionais, são as raizes da equação do segundo grau :

$$
\mathrm{X}^{2}+\mathrm{hX}+\mathrm{k}=0
$$

onde h é a curvatura média e k é a curvatura Gaussiana (escalar de Ricci) da conjunta. Essas curvaturas principais são valores máximos ou mínimos das curvaturas geométricas das curvas coordenadas.

Uma outra alternativa seria analisar as curvaturas de submodelos estatísticos (que seriam representados pelas distribuições perfiladas). Tal análise não é trivial do ponto de vista tensorial e envolve os chamados tensores de curvatura de Euler-Schouten (Amari,1982).

Nas páginas seguintes (figuras $5.10-5.13$ ) apresentamos t-plots e gráficos de verossimilhanças perfiladas referentes às duas reparametrizações. 

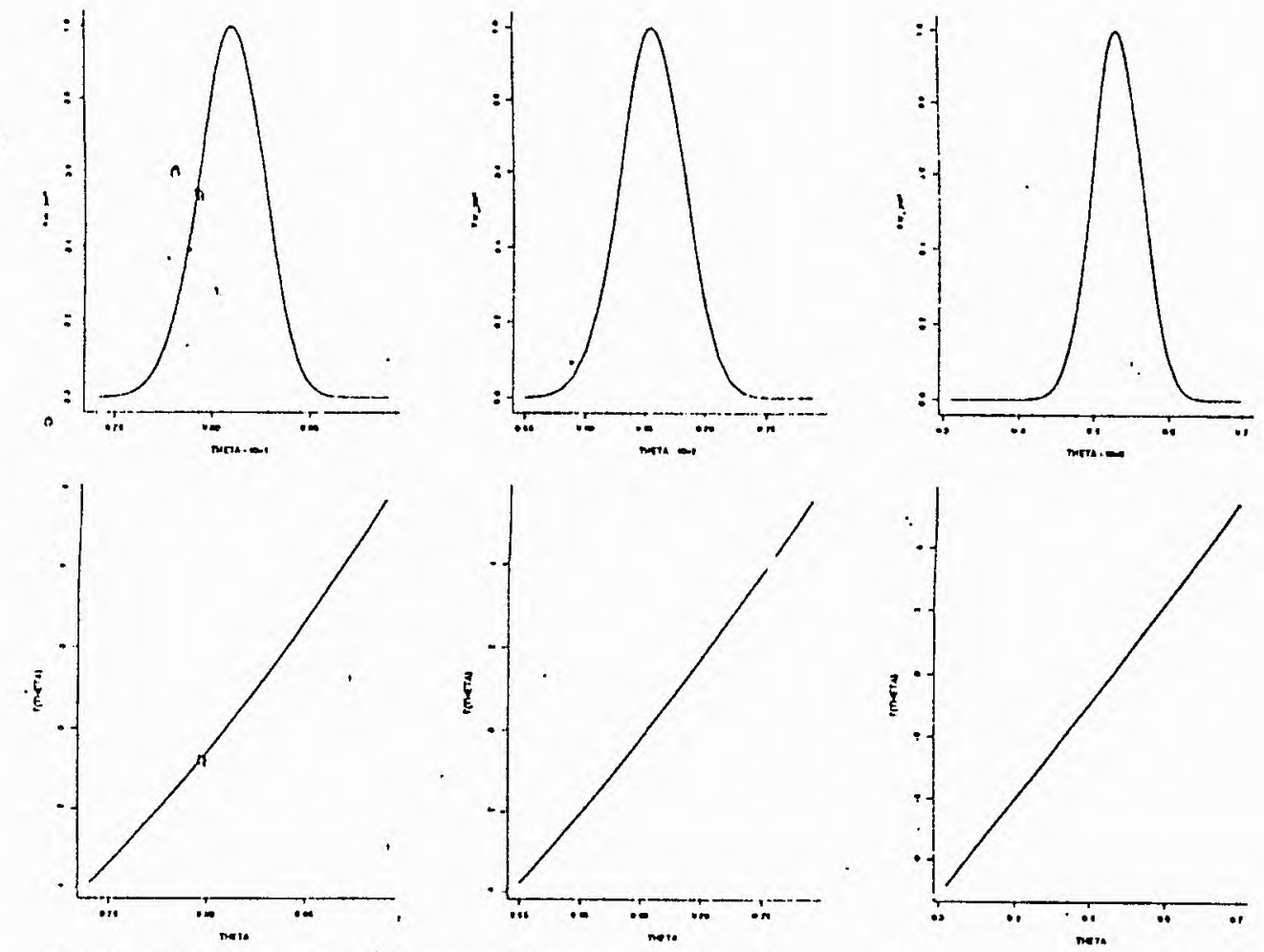

Figura 5.10. Gíráfico da verossimilhança perfilada para 00 com o respectivo "t-plot" nos tempos $l_{0}=1,2,3$ 

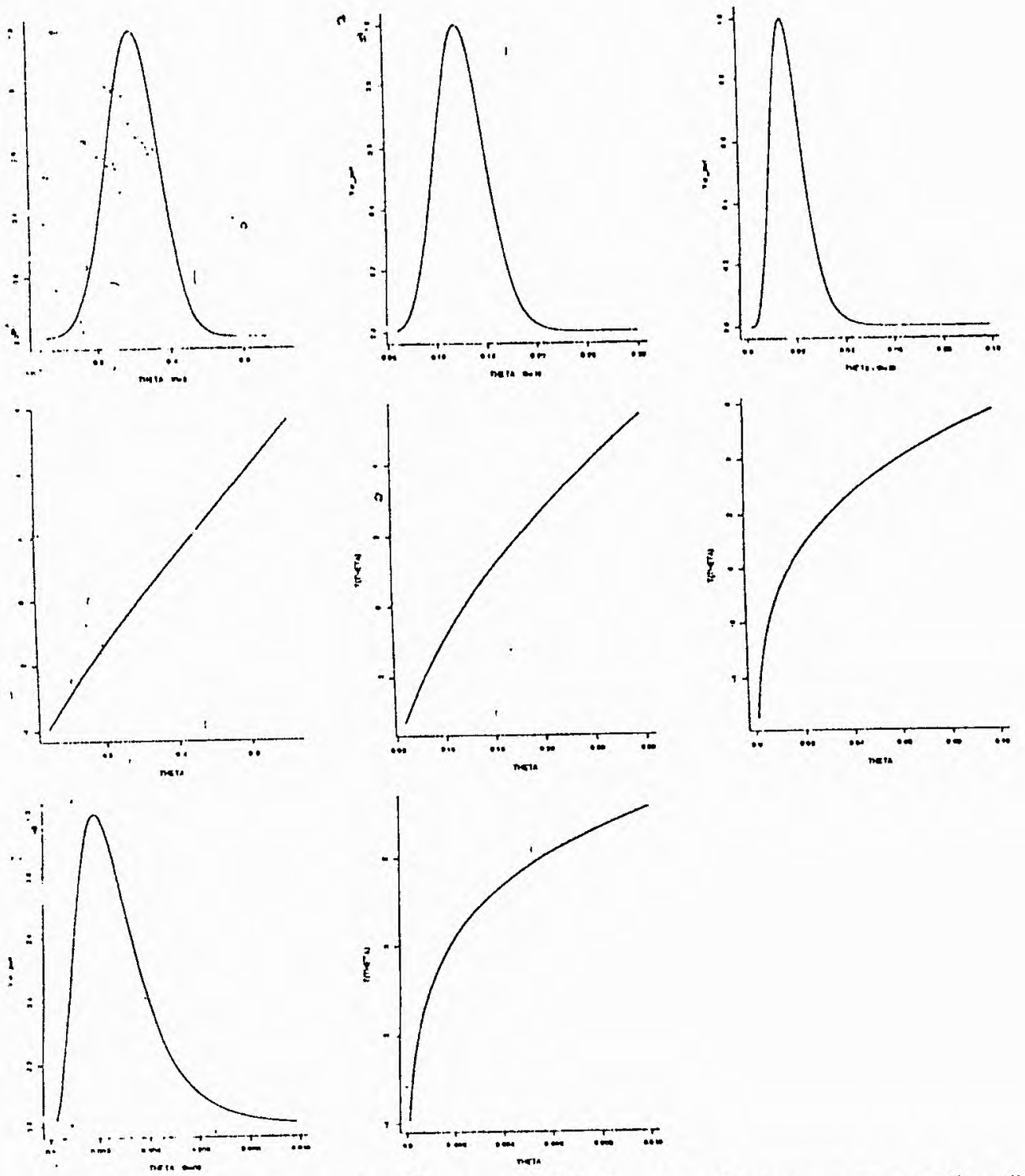

Figura 5.11. Ciráfico da verossimillıança perfilada para 00 com o respectivo "t-plot" nos tempos $t_{0}=5,10,20,30$ 

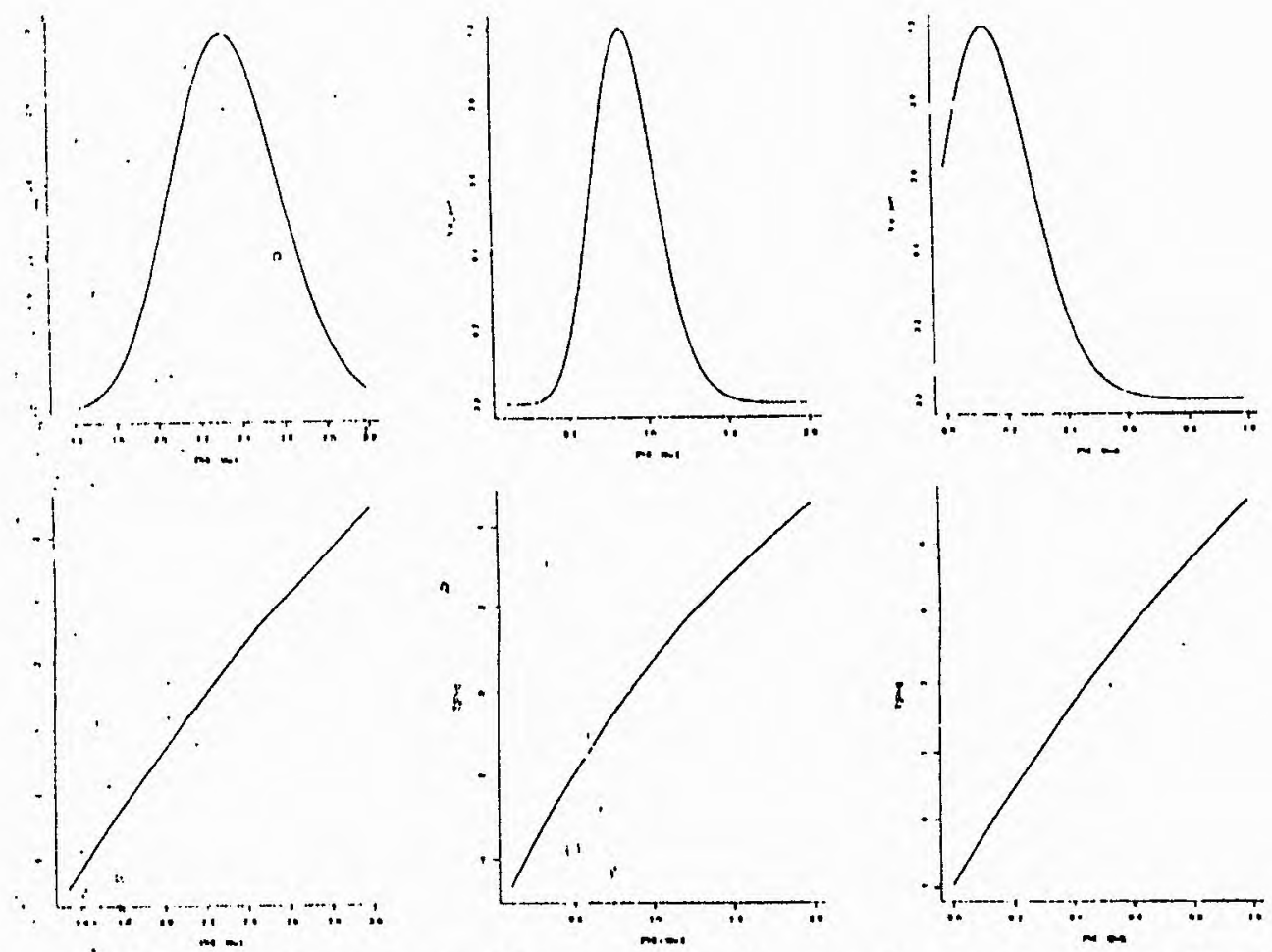

Figura 5.12. Cirálico da verossimillanģa perfilada para $\phi(j . l$ com o respectivo "t-plot" nes tompos $l_{10}=1,2,3$ 

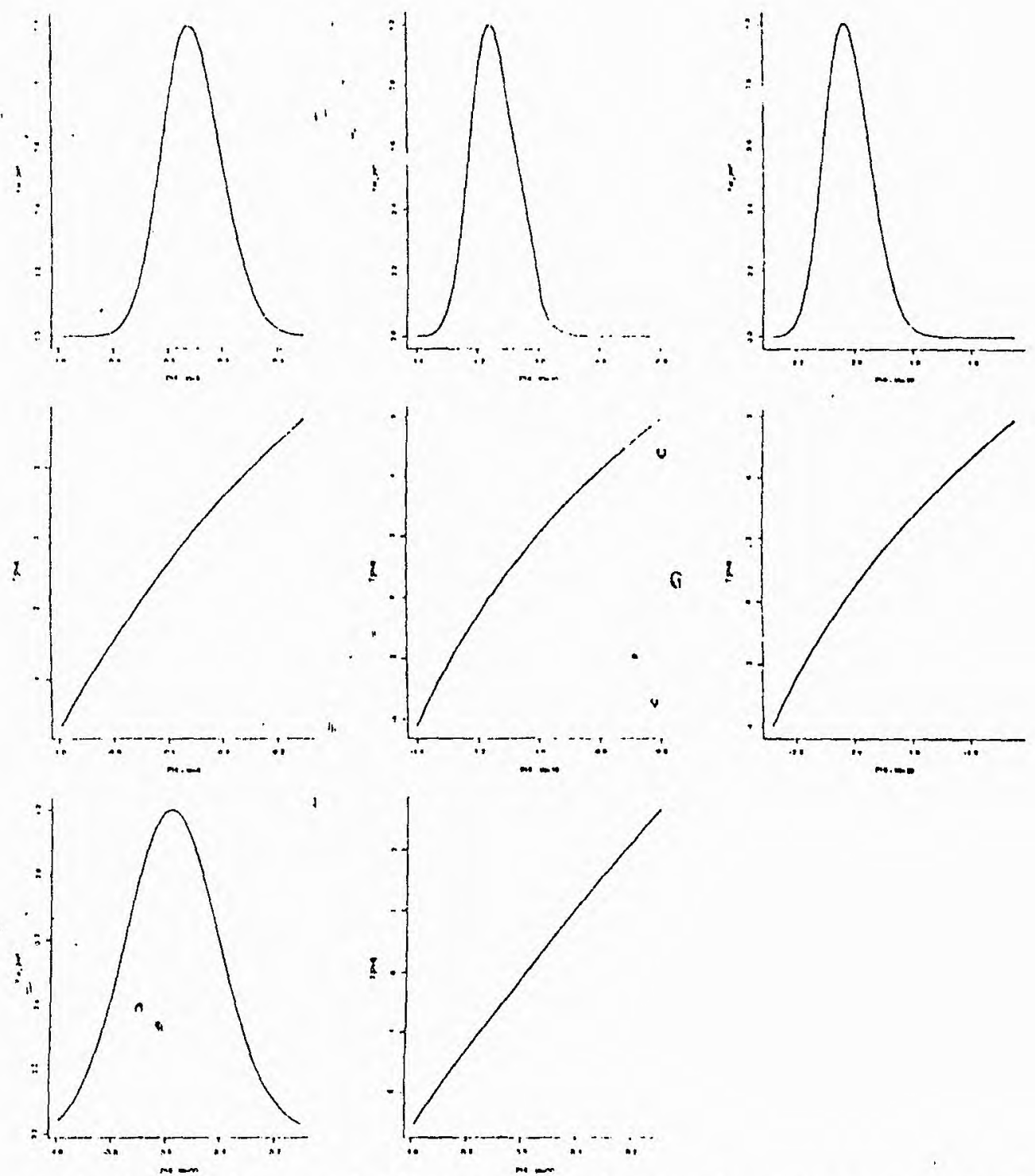

Figura 5.13. Gráfico da verossimilhança perfiladia para $\phi_{G . l}$ com o respectivo "l-plol" nos tcupos $l_{0}=5,10,20,30$ 


\section{Capítulo 6}

\section{Conclusões e Propostas Futuras}

Neste trabalho foi apresentada uma nova medida de curvatura para uso em modelos estatísticos. Estudou-se o seu comportamento através de vários exemplos numéricos e se demonstrou formalmente sua expressão matemática. Nos exemplos estudados ela se mostrou eficiente em detectar qual entre duas parametrizações apresentava comportamento mais próximo da normalidade e, portanto, assegurava que as inferências vindas dessa parametrização eram mais próximos dos resultados assintóticos. Esses resultados também são demonstrados pela medida de Kass e Slate e t plots de Hills.

Uma demonstração que tal fato é sempre verdadeiro: parametrização mais próxima da normalidade, escalar de Ricci menor poderia ser feita matematicamente em analogia a demonstração da medida de Kass e Slate (Slate, 1992). Mas tal demonstração exigiria a troca dos símbolos de Christofell's de primeira ordem pelos de segunda, o que exigiria um profundo estudo do significado estatístico de tais símbolos. Isso se enquadraria na conexão entre o cálculo tensorial e a Estatística, conexão essa ainda bastante recente.

Quanto a outros exemplos:

a) Pudemos provar que o escalar de Ricci não é capaz de captar a ortogonalização ou de parâmetros no sentido de Cox e Reid (1987)

b) Três exemplos envolvendo uso de distribuições a priori mostram que o escalar diminui com o uso de tais prioris em comparação com as respectivas distribuições da Estatística Clássica. A razão disso ocorrer é obscura e a questão é muito interessante pois poderia Ter grandes implicações nas pesquisas da escola Bayesiana de inferência, em especial envolvendo os algoritmos Gibbs-Sampler e Metrópolis-Hastings.

c) Em modelos não lineares a curvatura de Ricci pode indicar quão não linear é o modelo e se aproximações lineares são ou não confiáveis. Uma possível conexão com as curvaturas de Bates e Watts (Bates e Watts, 1980) poderia ser estabelecida.

d) Uma possível relação entre o escalar de Ricci da distribuição conjunta e das perfiladas envolve considerável algebrismo tensorial.

e) Melhores aproximações de Laplace associam-se a menores valores do escalar de Ricci. Isso é decorrência da melhor Normalidade de distribuições com melhores aproximações de Laplace.

Quanto às prespectivas futuras, podemos citar algumas:

a) Implementar um exemplo de MCMC onde um passo do algoritmo fosse canônico e outro teria o processo de escolha do próximo ponto pela curvatura.

b) Considerar se é possível obter um processo matemático para buscar a melhor parametrização usando álgebra tensorial.

c) Estudar conexão entre objetos estatísticos e objetos tensoriais da geometria diferencial, como, por exemplo, qual o significado do teorema de Bayes na geometria dos modelos estatísticos.

d) Explorar novos tipos de produtos internos nessas geometrias, baseados, por exemplo, em medidas de entropia e outros tipos de informação diferentes da de Fisher. 


\section{Apêndice 1}

\section{Vetores Contravariantes e Vetores Covariantes}

Todo conjunto de $\mathrm{n}$ quantidades $\mathrm{A}^{\mathrm{i}}$ que se transformam como suas diferenciais quando da troca arbitrária das coordenadas, é chamado vetor contravariante. Temos então, numa transformação de coordenadas

$$
A^{i}=\partial x^{i} / \partial x^{2 k} A^{, k}
$$

Designaremos as componentes dos vetores contravariantes por índices no alto das letras (sobreescrito).

Seja $\phi$ um escalar. As n quantidades $\partial \phi / \partial x^{i}$ se transformam numa mudança de coordenadas segundo as fórmulas

$$
\partial \phi / \partial \mathrm{x}^{\mathrm{k}}=\left[\partial \phi / \partial \mathrm{x}^{, \mathrm{k}}\right]\left[\partial \mathrm{x}^{\mathrm{k}} / \partial \mathrm{x}^{\mathrm{k}}\right] \text {, }
$$

Todo o conjunto de $n$ quantidades $A$ que se transformam nas mudanças de coordenadas como as derivadas de um escalar é chamado um vetor covariante. Assim, na mudança de coordenadas, temos

$$
\mathrm{A}_{\mathrm{i}}=\left[\partial \mathrm{x}^{\mathrm{k}} / \partial \mathrm{x}^{\mathrm{i}}\right] \mathrm{A}_{\mathrm{k}}^{\mathrm{i}},
$$
(subscrito).

As componentes dos vetores covariantes terão índices abaixo das letras

Num sistema de coordenadas cartesiano não há diferença entre vetores covariantes e contravariantes, as regras de transformação tornam-se equivalentes.

Nas coordenadas curvilineas há as duas espécies de vetores. Dessa forma há três espécies de tensores de segunda ordem. Chamamos de tensor contravariante de segunda ordem um conjunto $\mathrm{A}$ de $\mathrm{n}^{2}$ quantidades que se transformam como os produtos das componentes de dois vetores contravariantes, isto é, segundo a lei

$$
A^{i k}=\left[\partial x^{i} / \partial x^{\prime \prime}\right]\left[\partial x^{k} / \partial x^{\prime m}\right] A^{\prime l m}
$$

Definimos de maneira análoga um tensor covariante, que se tranformam segundo as regras

$$
A_{i k}=\left[\partial x^{\prime 1} / \partial x^{i}\right]\left[\partial x^{\prime m} / \partial x^{k}\right] A^{\prime} \operatorname{lm}
$$

e um tensor misto que se transforma como

$$
A_{k}^{i}=\left[\partial x^{i} / \partial x^{\prime l}\right]\left[\partial x^{\prime m} / \partial x^{k}\right] A_{m}^{\prime \prime}
$$

Os tensores de ordem superior têm definições semelhantes.

No sistema de coordenadas cartesiano podemos formar, a partir de dois vetores quaisquer, um escalar, o produto escalar desses vetores. Em coordenadas curvilíneas, não mais podemos formar um escalar com vetores quaisquer. Não podemos formar um 
escalar com dois vetores contravariantes ou covariantes, Com um vetor contravariante $A^{i}$ e um vetor covariante $B_{k}$ podemos formar um escalar: a quantidade $A^{k} B_{k}$ chamada produto escalar dos vetores $A^{k}$ e $B_{k}$. Pode-se provar, usando a definição de vetores contra e covariante, que $A^{\mathrm{k}} \mathrm{B}_{\mathrm{k}}$ é invariante nas transformações de coordenadas.

$O$ produto escalar de dois vetores é um caso particular de contração de tensores: se temos um tensor $\mathrm{A}^{\ldots . . . \ldots}$......, a expressão $\mathrm{A}^{\ldots . . . . .}$....... (soma sobre o índice i) é um tensor de ordem inferior de duas unidades em relação ao tensor $A^{. . . . . . . . . . . . . .}$

Para o tensor unitário em coordenadas curvilineas, temos a expressão $\delta^{\mathrm{k}}{ }_{\mathrm{i}}$ de componentes $=0$ para $i \neq k$ e 1 para $i=k$, Se $A^{k}$ é um vetor, contraindo em $\delta_{k}^{i}$ obtemos:

$$
\mathrm{A}^{\mathrm{k}} \delta_{\mathrm{k}}^{\mathrm{i}}=\mathrm{A}^{\mathrm{i}}
$$

isto é, temos de novo um vetor, o que prova ser $\delta_{i}^{\mathrm{k}}$ efetivamente um tensor. é,

O quadrado do elemento de arco $\mathrm{ds}^{2}$ é uma função quadrática das diferenciais $\mathrm{dx}^{\mathrm{i}}$, isto

$$
d s^{2}=g_{i j} d x^{i} d x^{j},
$$

onde os $g_{i j}$ são funções das coordenadas e simétricos: $g_{i j}=g_{j i j}$.

Dois tensores são inverso um do outro se

$$
\mathrm{A}_{\mathrm{ik}} \mathrm{B}^{\mathrm{kl}}=\delta_{\mathrm{i}}^{1}
$$

Chamamos de tensor métrico contravariante $\mathrm{g}^{\mathrm{ik}}$ o tensor que é o inverso de gik: $\mathrm{g}_{\mathrm{ik}} \mathrm{g}^{\mathrm{kl}}=\delta_{\mathrm{i}}^{\mathrm{l}}$.

\section{Derivação covariante}

Em coordenadas cartesianas as diferenciais $d A_{i}$ de uma vetor $A_{i}$ formam um vetor $e$ as derivadas parciais $\partial \mathrm{A}_{\mathrm{i}} / \partial \mathrm{x}^{\mathrm{k}}$ de suas componentes em relação às coordenadas, formam um tensor. Em coordenadas curvilíneas o mesmo não ocorre, $\mathrm{dA}_{\mathrm{i}}$ não é mais um vetor. Isto é devido ao fato de que $\mathrm{dA}_{\mathrm{i}}$ é a diferença de vetores que se encontram em pontos diferentes do espaço, os vetores se transformam diferentemente, pois os coeficientes nas fórmulas de transformação são funções das coordenadas.

Um vetor covariante se transforma como

$$
A_{i}=\left[\partial x^{\prime k} / \partial x^{k}\right] A_{k}^{\prime} \text {; }
$$

Consequentemente,

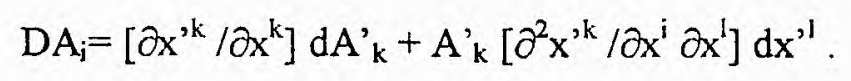

Então, os $\mathrm{dA}_{\mathrm{i}}$ não se transformam nunca como um vetor (o mesmo se aplica para diferenciais de vetores contravariantes). Somente no caso em que as derivadas segundas se anulam: $\left[\partial^{2} x^{k} / \partial x^{i} \partial x^{1}\right]=0$, isto é, os $x^{k k}$ são funções lineares dos $x^{k}$, é que as fórmulas têm a forma

$$
\mathrm{DA}_{\mathrm{i}}=\left[\partial \mathrm{x}^{\mathrm{k}} / \partial \mathrm{x}^{\mathrm{k}}\right] \mathrm{dA^{ \prime }}{ }_{\mathrm{k}}
$$

e os $\mathrm{dA}_{\mathrm{i}}$ se transformam como um vetor. 
A diferença $\mathrm{DA}^{\mathrm{i}}$ entre os vetores, que se encontram num mesmo ponto, é

$D A^{i}=d A^{i}-\delta A^{i}$,

onde $\delta \mathrm{A}^{\mathrm{i}}$ é o acréscimo dos componentes do vetor no transporte paralelo.

Sejam $A_{i}$ e $B^{i}$ vetores covariante e contravariante respectivamente. Um escalar deve ser invariante por transporte paralelo, dessa forma $\delta\left(A_{i} B^{i}\right)=0$,

$\delta A^{i}=-\Gamma_{k l}^{i} A^{k} d x^{l}, \quad$ (definição de $\delta A^{i}$ )

$B^{i} \delta A_{i}=-A_{i} \delta B^{i}=\Gamma_{k l}^{i} B^{k} A_{i} d x^{1}$

ou, trocando os índices $\mathrm{k} \mathrm{e} \mathrm{i,}$

$B^{i} \delta A_{i}=\Gamma^{k}{ }_{i l} A_{k} B^{i} d x^{1}$.

Como os $\mathrm{B}^{\mathrm{i}}$ são arbitrários:

$\delta A_{i}=\Gamma^{k}{ }_{i l} A_{k} d x^{\prime}$, paralelo.

o que determina a variação das componentes de um vetor covariante no transporte

Usando $\delta A^{i}=-\Gamma_{k l}^{i} A^{k} d x^{1}$ e $d A^{i}=\left[\partial A_{i} / \partial x^{1}\right] d x^{1}$, temos

$D A^{i}=\left(\left[\partial A^{i} / \partial x^{1}\right]+\Gamma_{k\rfloor}^{i} A^{k}\right) d x^{1}$,

De modo análogo, para um vetor covariante, temos

$D A_{i}=\left(\left[\partial A_{i} / \partial x^{l}\right]-\Gamma_{i l}^{k} A_{k}\right) d x^{l}$

As expressões entre parênteses são tensores, pois seus produtos pelo vetor $\mathrm{dx} \mathrm{x}^{\mathfrak{}} \mathrm{dão}$ um vetor. Elas desempenham em coordenadas curvilíneas o mesmo papel do tensor cartesiano $\partial A_{i} / \partial x^{k}$. Esses tensores são chamados de derivadas covariantes respectivas dos vetores $A^{i} e$ $A_{i}$. São designados por $A_{; k}^{i}$ e $A_{i ; k}$. Desse modo,

$$
\begin{aligned}
& D^{i}=\left(A_{; 1}^{i}\right) d x^{1} \\
& D_{i}=\left(A_{i ; 1.1}\right) d x^{1}
\end{aligned}
$$

Em coordenadas cartesianas, $\Gamma_{\mathrm{kl}}^{\mathrm{i}}=0$, e as derivadas covariantes coincidem com as derivadas ordinárias.

De forma semelhante, definimos a derivada covariante de um tensor. Para isso, devemos determinar como varia o tensor no transporte paralelo de um ponto para outro infinitamente próximo. Consideremos, por exemplo, um tensor contravariante arbitrário que é produto de dois vetores contravariantes $A^{i} B^{k}$. No transporte paralelo, temos:

$$
\delta\left(A^{i} B^{k}\right)=A^{i} \delta B^{k}+B^{k} \delta A^{i}=-A^{i} \Gamma_{l m}^{k} B^{l} d x^{m}-B^{k} \Gamma_{l m}^{i} A^{l} d x^{m}
$$


$A^{i k}$ :

Em virtude da linearidade dessa transformação, ela deve ser válida para todo tensor

$\delta A^{i k}=-\left(A^{i m} \Gamma_{m l}^{k}+A^{m k} \Gamma_{m l}^{i}\right) d x^{1}$

Substituindo essa expressão em

$D A^{i k}=d A^{i k}-\delta A^{i k}=A^{i k}, 1 x^{1}$,

Temos a derivada covariante do tensor $A^{i k}$ na forma

$$
A_{; 1}^{i k}=\left[\partial A^{i k} / \partial x^{l}\right]+\Gamma_{m l}^{i} A^{i n k}+\Gamma_{m l}^{k} A^{i m}
$$

De modo análogo, encontramos as derivadas covariantes de tensores mistos e covariantes sob a forma

$$
\begin{aligned}
& A_{k ; l}^{i}=\left[\partial A_{k}^{i} / \partial x^{l}\right]-\Gamma_{k l}^{m} A_{m}^{i}+\Gamma_{m l}^{i} A_{k}^{m} \\
& A_{i k ; l}=\left[\partial A_{i k} / \partial x^{l}\right]-\Gamma_{i l}^{m_{i l}} A_{m k}-\Gamma_{k l}^{m} A_{i m}
\end{aligned}
$$

Vamos mostrar que os símbolos de Christoffel $\Gamma_{\mathrm{k} \text { I }}^{\mathrm{i}}$ são simétricos em relação aos indices inferiores:

A derivada covariante $A_{i ; k}$ de um vetor sendo um tensor, segue-se que a diferença $A_{i ; k}$ $A_{k ; i}$ é também um tensor. Suponhamos que o vetor $A_{i}$ seja o gradiente de um escalar, isto é, que $\mathrm{A}_{\mathrm{i}}=\left[\partial \phi / \partial \mathrm{x}^{\mathrm{i}}\right]$. Como

$$
\partial A_{i} / \partial x^{k}=\left[\partial^{2} \phi / \partial x^{i} \partial x^{k}\right]=\partial A_{k} / \partial x^{i}
$$

Usando

$$
A_{i j, l}=\left(\left[\partial A_{i} / \partial x^{l}\right]-\Gamma^{k}{ }_{i l} A_{k}\right)
$$

Para $A_{i ; k}$,

$$
A_{k, i}-A_{i ; k}=\left(\Gamma_{i k}^{1}-\Gamma_{k i}^{1}\right)\left[\partial \phi / \partial x^{\prime}\right]
$$

Em coordenadas cartesianas as derivadas covariantes coincidem com as derivadas ordinárias, e o primeiro membro da igualdade acima é nulo. Mas como $A_{k ; i}-A_{i ; k}$ é um tensor, se ele se anula num sistema de coordenadas, o mesmo deve ocorrer em qualquer outro sistema.

Deduzimos que

$$
\Gamma_{\mathrm{kl}}^{\mathrm{i}}=\Gamma_{1 \mathrm{k}}^{\mathrm{i}}
$$

De modo análogo,

$$
\Gamma_{\mathrm{i}, \mathrm{kl}}=\Gamma_{\mathrm{i}, \mathrm{lk}}
$$

\section{Ligacão entre os símbolos de Christoffel e o tensor métrico}

Vamos mostrar que a derivada covariante do tensor métrico gik é nula. Notemos para isso que devemos ter para o vetor $\mathrm{DA}_{i}$, como para todo vetor, a relação 
$D A_{i}=g_{i k} D A^{k}$

Por outro lado, $A_{i}=g_{i k} A^{k}$, de modo que

$D A_{i}=D\left(g_{i k} A^{k}\right)=g_{i k} D A^{k}+A^{k} D g_{i k}$

Comparando com $D A_{i}=g_{i k} D A^{k}$, obtemos, sabendo que o vetor $A^{i}$ é arbitrário:

$\operatorname{Dg}_{i k}=0$.

Resulta então para a derivada covariante

$\mathrm{gik}_{\mathrm{i}}=0$. covariante.

Conseqüentemente, deve-se considerar os g $_{i k}$ como constantes sob derivação

Nos serviremos da igualdade $\mathrm{g}_{\mathrm{ik} ; \mathrm{i}}=0$ para exprimir os símbolos de Christoffel $\Gamma_{\mathrm{k} l}^{\mathrm{i}}$ por meio do tensor gik. Temos:

$$
\begin{aligned}
& A_{i k ; l}=\left[\partial A_{i k} / \partial x^{1}\right]-\Gamma^{m} A_{m k}-\Gamma_{k l}^{m} A_{i m} \\
& g_{i k ; l}=\left[\partial g_{i k} / \partial x^{1}\right]-\Gamma^{m}{ }_{i l} g_{m k}-\Gamma_{k l}^{m} g_{i m}=\left[\partial g_{i k} / \partial x^{i}\right]-\Gamma_{k i l}-\Gamma_{i k l} \\
& \text { Mas } \\
& g_{i k ; l}=0
\end{aligned}
$$

Conseqüentemente, as derivadas dos $\mathrm{g}_{\mathrm{ik}}$ se exprimem por meio dos símbolos de Christoffel. Escrevamos essas derivadas, permutando circularmente os índices $\mathrm{i}, \mathrm{k}, \mathrm{l}$ :

$\left[\partial \mathrm{g}_{\mathrm{ik}} / \partial \mathrm{x}^{1}\right]=\Gamma_{\mathrm{kil}}+\Gamma_{\mathrm{ik}]}$

$\left[\partial \mathrm{g}_{\mathrm{li}} / \partial \mathrm{x}^{\mathrm{k}}\right]=\Gamma_{\mathrm{ik} l}+\Gamma_{\mathrm{ik}}$

$-\left[\partial \mathrm{g}_{\mathrm{kl}} / \partial \mathrm{x}^{\mathrm{i}}\right]=-\Gamma_{\mathrm{iki}}-\Gamma_{\mathrm{kli}}$

Tomando-se a semi-soma dessas igualdades e usando a propriedade $\Gamma_{\mathrm{ikl}}=\Gamma_{\mathrm{ilk}}$, temos :

$\Gamma_{\mathrm{i}, \mathrm{kl}}=1 / 2\left(\partial \mathrm{g}_{\mathrm{ik}} / \partial \mathrm{x}^{\mathrm{l}}+\partial \mathrm{g}_{\mathrm{il}} / \partial \mathrm{x}^{\mathrm{k}}-\partial \mathrm{g}_{\mathrm{k} l} / \partial \mathrm{x}^{\mathrm{i}}\right)$

De onde, deduzimos para os $\Gamma_{\mathrm{kl}}^{\mathrm{i}}=\mathrm{g}^{\mathrm{im}} \Gamma_{\mathrm{m}, \mathrm{kl}}$ :

$$
\Gamma_{\mathrm{kl}}^{\mathrm{i}}=1 / 2 \mathrm{~g}^{\mathrm{im}}\left(\partial \mathrm{g}_{\mathrm{mk}} / \partial \mathrm{x}^{\mathrm{l}}+\partial \mathrm{g}_{\mathrm{m} l} / \partial \mathrm{x}^{\mathrm{k}}-\partial \mathrm{g}_{\mathrm{k}} / \partial \mathrm{x}^{\mathrm{m}}\right)
$$

Tais são as fórmulas que dão as expressões procuradas dos símbolos de Christoffel em função do tensor métrico.

\section{O tensor de Curvatura}

Estabeleçamos a fórmula geral que determina a variação de um vetor durante seu transporte paralelo ao longo de um contorno infinitesimal fechado. Essa variação $\Delta \mathrm{A}_{\mathrm{k}}$ pode ser expressa sob a forma $\int \mathrm{dA}_{\mathrm{k}}$, onde a integral é tomada sobre o contorno dado.

Logo

$$
\Delta \mathrm{A}_{\mathrm{k}}=\int \Gamma_{\mathrm{kl}}^{\mathrm{i}} \mathrm{A}_{\mathrm{i}} \mathrm{dx}
$$


sendo que o vetor $A_{i}$ no integrando varia durante seu transporte ao longo do contorno. Aplicando o teorema de Stokes à integral e observando que a área delimitada pelo contorno considerado é uma quantidade infinitesimal $\Delta \mathrm{f}^{\mathrm{m}}$, obtemos:

$\Delta \mathrm{A}_{\mathrm{k}}=1 / 2\left[\partial\left(\Gamma_{\mathrm{km}}^{\mathrm{i}} \mathrm{A}_{\mathrm{i}}\right) / \partial \mathrm{x}^{1}-\partial\left(\Gamma_{\mathrm{kl}}^{\mathrm{i}} \mathrm{A}_{\mathrm{i}}\right) / \partial \mathrm{x}^{\mathrm{m}}\right] \Delta \mathrm{f}^{\mathrm{dm}}$

$\Delta A_{k}=1 / 2\left[A_{i} \partial\left(\Gamma_{k m}^{i}\right) / \partial x^{1}-A_{i} \partial\left(\Gamma_{k l}^{i}\right) / \partial x^{m}+\Gamma_{k m}^{i} \partial A_{i} / \partial x^{1}++\Gamma_{k l}^{i} \partial A_{i} / \partial x^{m}\right] \Delta f^{d m}$

Substituindo nessa última expressão os valores das derivadas, deduzidas de $\partial A_{i} / \partial x^{\prime}=\Gamma_{i 1}^{n} A_{n}$

deduzimos

$\Delta A_{k}=1 / 2 R_{k l m}^{i} A_{i} \Delta f^{d m}$

onde $\mathrm{R}_{\mathrm{kmm}}^{\mathrm{i}}$ é um tensor de quarta ordem:

$\mathrm{R}_{\mathrm{klm}}^{\mathrm{i}}=\partial \Gamma_{\mathrm{km}}^{\mathrm{i}} / \partial \mathrm{x}^{\mathrm{l}}=\partial \Gamma_{\mathrm{kl}}^{\mathrm{i}} / \partial \mathrm{x}^{\mathrm{m}}+\Gamma_{\mathrm{nl}}^{\mathrm{i}} \Gamma_{\mathrm{km}}^{\mathrm{n}}-\Gamma_{\mathrm{mml}}^{\mathrm{i}} \Gamma_{\mathrm{kl}}^{\mathrm{n}}$

$\mathrm{O}$ tensor $\mathrm{R}_{\mathrm{klm}}^{\mathrm{i}}$ é chamado tensor de curvatura ou tensor de Riemann.

O tensor de curvatura é nulo no espaço euclidiano. Isso se justifica pelo fato de termos um sistema de coordenadas onde $\Gamma_{\mathrm{kl}}^{\mathrm{i}}=0$ o que acarreta $\mathrm{R}_{\mathrm{klm}}^{\mathrm{i}}=0$. Em virtude do caráter tensorial de $\mathrm{R}_{\mathrm{klm}}^{\mathrm{i}}$, ele será nulo em qualquer outro sistema de coordenadas. A razão geométrica desse fato é que num espaço euclidiano o transporte paralelo de um vetor de um ponto a outro é uma operação univoca, na qual o vetor não varia durante seu transporte ao longo de um contorno fechado.

A recíproca também é verdadeira: se $R_{\mathrm{klm}}^{i}=0$, o espaço é euclidiano. Com efeito, podemos escolher em todo espaço um sistema de coordenadas cartesianas num domínio infinitesimal dado ao domínio vizinho, e assim por diante, podemos construir um sistema cartesiano em todo o espaço, o que mostra o caráter euclidiano do espaço.

Conseqüentemente, o fato do tensor de curvatura ser nulo ao não é um critério que permite reconhecer se o espaço é ou não euclidiano. 


\section{Apêndice 2}

\section{Expressões dos tensores para 0 caso bidimensional geral $\mathbf{f}=\mathbf{f}\left(\theta_{1}, \theta_{2}\right)$}

(i)Christofell de primeira espécie

$\Gamma_{\mathrm{ijk}}=1 / 2 f_{\mathrm{ijk}}=1 / 2 \partial^{2} \mathrm{f} / \partial \theta_{\mathrm{i}} \partial \theta_{\mathrm{j}} \partial \theta_{\mathrm{k}}$

$\mathrm{i}, \mathrm{j}, \mathrm{k}=1,2$

(ii)Christofell de segunda espécie

$\Gamma_{11}^{1}=1 /(2 \Delta)\left[f_{22} f_{111}-f_{112} f_{12}\right]$

$\Gamma_{12}^{1}=\Gamma_{21}^{1}=1 /(2 \Delta)\left[f_{22} f_{112}-f_{122} f_{12}\right]$

$\Gamma^{1}{ }_{22}=1 /(2 \Delta)\left[f_{22} f_{122}-f_{111} f_{12}\right]$

$\Gamma_{11}^{2}=1 /(2 \Delta)\left[f_{22} f_{111}-f_{112} f_{12}\right]$

$\Gamma_{12}^{1}=\Gamma_{21}^{1}=1 /(2 \Delta)\left[f_{11} f_{122}-f_{112} f_{12}\right]$

$\Gamma_{22}^{2}=1 /(2 \Delta)\left[f_{11} f_{111}-f_{122} f_{12}\right]$

$\Delta=\operatorname{det}(\mathrm{G})=\mathrm{f}_{11} \mathrm{f}_{22}-\mathrm{f}_{12} \mathrm{f}_{12}$

$R_{\mathrm{ijkm}}=\left(\partial_{\mathrm{i}} \Gamma_{\mathrm{jk}}^{\mathrm{s}}-\partial_{\mathrm{j}} \Gamma_{\mathrm{ik}}^{\mathrm{s}}\right) \mathrm{g}_{\mathrm{sm}}+\left(\Gamma_{\mathrm{irm}} \Gamma_{\mathrm{jk}}^{\mathrm{r}}-\Gamma_{\mathrm{j} m} \Gamma^{\mathrm{r}} \mathrm{ik}\right)$

$R_{1212}=1 /(4 \Delta)\left[f_{22}\left(f_{111} f_{122}-f_{112}^{2}\right)+f_{12}\left(f_{122} f_{112}-f_{111} f_{222}\right)+f_{11}\left(f_{222} f_{122}-f^{2}{ }_{112}\right)\right]$

$\mathrm{R}_{1212}=1 / 4 \operatorname{det}(\mathrm{M}) / \operatorname{det}(\mathrm{G})$

Onde $\mathrm{G}$ é a matriz de Fisher e $\mathrm{M}$ é uma matriz $3 \times 3$ :

$M(1,1)=f_{11}$

$M(1,2)=f_{12}$

$\mathrm{M}(1,3)=\mathrm{f}_{22}$

$\mathrm{M}(2,1)=\mathrm{f}_{111}$

$M(2,2)=f_{121}$

$M(2,3)=f_{221}$

$M(1,1)=f_{112}$

$M(1,2)=f_{122}$

$\mathrm{M}(1,3)=\mathrm{f}_{222}$

$K=-g^{11} g^{22} R_{1212}=-1 / 4 \quad g_{11} g_{22} \operatorname{det}(M) / \operatorname{det}(G)$

$\mathrm{K}$ é a contração dupla de $\mathrm{R}_{1212}$, tensor de Riemann, e é chamado curvatura escalar de Ricci. 


\section{Apêndice 3 \\ Método de Laplace para aproximação de Integrais}

Suponha que temos interesse em integrais da forma:

$I=\int f(\theta) \exp [-n h(\theta)] d \theta$

Onde $h(\theta)$ é uma função unimodal com máximo em $\theta^{+}$.

Para casos uniparamétricos $(\theta \in R)$, se $\theta^{+}$é o valor que maximiza $-\mathrm{h}(\theta)$ e $\sigma=\left[-\mathrm{h}^{\prime \prime}\right.$ $\left.\left(\theta^{+}\right)\right]^{1 / 2}$, então o método de Laplace estabelece a seguinta aproximação para a integral I:

$I \sim(2 \pi / n)^{1 / 2} \sigma f\left(\theta^{+}\right) \exp \left[-\mathrm{nh}\left(\theta^{+}\right)\right]$

Para casos multiparamétricos, $\left(\theta \in \mathrm{R}^{\mathrm{n}}\right)$, a aproximação é dada por

$\mathrm{I} \sim(2 \pi)^{\mathrm{n} / 2}\left[\operatorname{det}\left(\mathrm{nD} \mathrm{D}^{2} \mathrm{~h}\left(\theta^{+}\right)\right)\right]^{-1 / 2} \mathrm{f}\left(\theta^{+}\right) \exp \left[-\mathrm{nh}\left(\theta^{+}\right)\right]$

Onde o vetor $\theta$ maximiza a matriz $-h(\theta)$ e $D^{2} h\left(\theta^{+}\right)$é a matriz hessiana de $h\left(\theta^{+}\right)$ calculada em $\theta^{+}$. 


\section{Apêndice 4 \\ Medidas de Curvaturas de Bates e Watts e profiles t-plots}

Bates e Watts (1980) provaram que a não linearidade de um modelo pode ser decomposta em duas componentes : a não linearidade intrínseca (IN) e a não linearidade devido ao efeito dos parâmetros ( $\mathrm{PE}$ ). Para um modelo não linear, o espaço de estimação é curvo e (IN) mede a extensão dessa curvatura. É comum o uso de aproximações lineares para tais modelos, tais aproximações só são possiveis para valores baixos de (IN), conforme discutido em Bates e Watts (1980).

A não linearidade devido ao efeito de parâmetros é medida por $(\mathrm{PE})$ e é consequência da falta de uniformidade do sistema de coordenadas no espaço paramétrico. IN é invariante sob reparametrização, $\mathrm{PE}$ não. Valores altos dos arrays de curvatura de Bates e Watts indicam maior não linearidade, a reparametrização pode contribuir para diminuir os valores do array de curvatura associado a (PE).

Outra forma de se avaliar a extensão do comportamento não linear é através dos chamados profiles t-plots (Ratkowsky, 1990). Os profiles t-plots revelam graficamente a extensão da não linearidade para cada parâmetro. Comportamento linear do parâmetro associa-se a t-plots retilíneos e não linearidade por t-plots não retilineos. 


\section{Apêndice 5 \\ Medidas de Não linearidade}

No caso uniparamétrico a terceira derivada padronizada da função logverossimilhança foi proposta por Sprott (1973) e é expressa por:

$$
\operatorname{STD}(\beta)=\left|1_{\beta \beta \beta}\left(\beta^{+}\right) 1_{\beta \beta}\left(\beta^{+}\right)^{-3 / 2}\right|
$$

Caso o valor encontrado seja próximo de zero afirma-se que a normalidade assintótica do estimador de máxima verossimilhança é satisfatória. Uma generalização da terceira derivada padronizada da função log-verossimilhança, para o caso multiparamétrico, é dada pela medida global de não-normalidade de Kass e Slate (1992):

$$
B^{2}(\theta)=\sum_{a, b, c, d, e, f} d_{a b c} d_{d c f} b_{a b} b_{d e} b_{c f},
$$

Onde $b_{m n}$ são os elementos da inversa da matriz de Fisher e $d_{x y z}=\partial 1(\theta) / \partial \theta_{x} \partial \theta_{y} \partial \theta_{z}$. De maneira análoga ao caso unidimensional, a boa normalidade da distribuição conjunta se verifica para baixos valores de $\mathrm{B}^{2}$.

Outro indicativo da não-normalidade é o t-plot de Hills e Smith. Trata-se de uma maneira gráfica: distribuições próximas da normalidade apresentam t-plot de Hills e Smith retilineos, distribuições afastadas da normalidade apresentam t-plots de Hills e Smith curvos. 


\section{Referências}

Achcar, J. A. e Smith,A. F.M.(1990). Aspects of Reparametrization in Approximative Bayesian Inference, Geisser, S.Hodges, J.S., Press, S.J.Zellner, A.(Editors), Bayesian and Likehood Methods in Statistics and Econometrics: Essays in Honor of George A Barnard, Elservier Science Publisher, BV (North-Holland)

Amari, S.I. (1982). Differential Geometry of Curved Exponential families- Curvatures and Information Loss, Ann. Statist, 10,375-385.

Anscombe, F.J. (1964). Normal Likehood Functions, Annals of the Institute of Statistical Mathematics, 16,1-19.

Bates, D.M. e Watts, D.G. (1980). Relative curvature measures of nonlinearity, J.Roy.Statist.Soc.B40,1-25.

Bates, D.M. e Watts, D.G. (1988). Nonlinear Regression Analysis and Its Applications, Wiley, New York.

Barndorff-Nielsen, O E.(1986). Likehood and observed geometries. Ann. Statist. 14, 856-873.

Box, M.J. (1971). Bias in Nonlinear Estimation, Journal of the Royal Statistical Society, Series B,33, 171-201.

Box, G.E.P. e G.C. Tiao(1973). Bayesian Inference in Statistical Analysis, Addinson-Wesley.

Carr, N.L. (1960), Kinetics of catalytic isomerization of npentane. Industrial and Engineering Chemistry, 52,391-396.

Cox, D.R. e N. Reid (1987).Parameter Orthogonality and Approximative Conditional Inference, J.R.Statist.Soc.,B, Vol.49, N 1,1-39.

Gelman, A. E. e Rubin, D. (1992) Inference from iterative simulation using multiple sequences, Statistical Science, 7, 457-472,1992.

Heyes, K.K., Brown R. (1956) Growth and Celular Differential in FL Milthorpe (ED.) The Growth of Leaves, Butterworth, London

Hills, S.(1989).The Parameterisation of Statistical Models, Ph.D.Thesis, University of Nottingham.

Hills, S.E; Smith, A F.M.(1993). Diagnostics of Posteriori Nonnormality in Bayesian Inference. Biometrica 80, 1, 61-74.

Kass, R.E., Slate, E.H.(1992). Reparameterization of Statistical Models, Tese de Doutorado, Department of Statistics, Carnegie Mellon University, Pittsburgh, PA.

Leandro, R.A.(1997). Uso de Métodos Bayesianos na Análise de Dados de Tempo de Sobrevivência Bivariados, Tese de Doutorado, Esalq, USP.

Ratkowsky, D. A. (1990). Handbook of Nonlinear Regression Models, Marcel Dekker, New York. 
Seber, G.A .F. e Wild, C.J.(1989).Nonlinear Regression,.John Wiley \& Sons, New York.

Sprott, D.A.(1973).Normal likehoods and their relation to large sample theory of estimation,Biometrika,60,457-465.

Sprott, D.A (1980). Maximum Likehood in Small Samples: Estimation in the Presense of Nuisance Parameters, Biometrika, 67, 515-523. 